\title{
In situ structural and compositional analysis using RHEED electrons induced $\mathrm{x}$-rays
}

\author{
Sandeep Chandril \\ West Virginia University
}

Follow this and additional works at: https://researchrepository.wvu.edu/etd

\section{Recommended Citation \\ Chandril, Sandeep, "In situ structural and compositional analysis using RHEED electrons induced x-rays" (2009). Graduate Theses, Dissertations, and Problem Reports. 2878. \\ https://researchrepository.wvu.edu/etd/2878 \\ This Dissertation is protected by copyright and/or related rights. It has been brought to you by the The Research Repository @ WVU with permission from the rights-holder(s). You are free to use this Dissertation in any way that is permitted by the copyright and related rights legislation that applies to your use. For other uses you must obtain permission from the rights-holder(s) directly, unless additional rights are indicated by a Creative Commons license in the record and/ or on the work itself. This Dissertation has been accepted for inclusion in WVU Graduate Theses, Dissertations, and Problem Reports collection by an authorized administrator of The Research Repository @ WVU. \\ For more information, please contact researchrepository@mail.wvu.edu.}




\title{
In situ structural and compositional analysis using RHEED electrons induced $x$-rays
}

\author{
Sandeep Chandril
}
Dissertation submitted to the Eberly College of Arts and Sciences at West Virginia University in partial fulfillment of the requirements
for the degree of
Doctor of Philosophy
in
Physics

\author{
David Lederman, Ph.D., Chair \\ Thomas H. Myers, Ph.D. \\ Mohindar Seehra, Ph.D. \\ Sergei Urazhdin, Ph.D. \\ Xian-An Cao, Ph.D.
}

Department of Physics

Morgantown, West Virginia

2009

Keywords:

X-ray fluorescence (XRF), RHEED-TRAXS, Reciprocity theorem,

Parratt's Formalism, CASINO 


\section{ABSTRACT \\ RHEED-TRAXS as a thin film structural and compositional analysis \\ technique}

\section{Sandeep Chandril}

The use of x-ray fluorescence (XRF) for in-situ chemical analysis of a sample surface using an electron gun for reflection high energy electron diffraction (RHEED) analysis has been studied by many groups and the technique is usually referred to as RHEED-total-refection-angle $\mathrm{x}$-ray spectroscopy (RHEED-TRAXS), as the angle for $\mathrm{x}$ ray detection was set close to the angle of total internal reflection.

In the current work, RHEED-TRAXS was developed as a thin film structural characterization technique based on the principles similar to the analysis of a multilayered structure by angular-dependent total-reflection x-rays. Initial experiments utilizing the technique for relative compositional analysis have also been discussed.

Utilizing RHEED-TRAXS as a structural and compositional analysis technique has numerous benefits. In-situ determination of thickness and roughness of film(s) yields information that could be destroyed due to atmospheric exposure and compositional analysis can be used to study and control the growth parameters.

Since a RHEED electron beam gun is a common feature on MBE systems, the addition of a relatively inexpensive x-ray detector on a translation stage adds the capability of exhaustive in-situ analysis where growth parameters can be studied and controlled to a greater degree. 


\section{Acknowledgements}

Foremost, I would like to thank my advisor, Dr. David Lederman, for his constant guidance and support. His role has been instrumental in every aspect of the project. He has pulled me out of many tight situations and has maintained a positive attitude throughout. I would also like to thank Dr. Tom Myers for his guidance for the thesis

project and, especially, during the early graduate years. Though a lot of work done with Dr. Myers couldn't be a part of this thesis because of totally disjoint topics, it helped me immensely as a researcher. I would also like to thank the other members of my committee for their support and taking the time out of their busy schedules to serve on my committee.

I would also like to thank my colleagues, Randy Tompkins, Cameron Keenan and Eric Schires for helping me on countless occasions. Randy, Cameron and Eric helped install the RHEED-TRAXS arm on the oxide system. Randy and Cameron helped perform the initial experiments and Cameron has assisted me with every aspect of the research from growth to analysis. I extend my sincerest thanks to Randy and Cameron for numerous discussions, valuable feedbacks and moral support.

I would also like to thank Eric Schires, Felio Perez, Carl Weber, and Doug Matthes for their technical assistance that made the experiments possible. Also Sherry Puskar, Sandy Jones and Siobhan Byrne for doing all the paperwork for us. Thanks to Lillyan Dylla and Phillip Staib from Staib Instruments Inc. for their patience, cooperation and technical support that was vital for the success of the experiments.

Most of all, I am grateful to my family and my parents for all the love and support, and believing in me. For that, I am forever indebted to them. 


\section{Table of Contents}

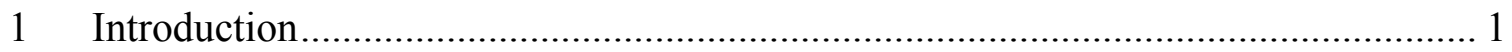

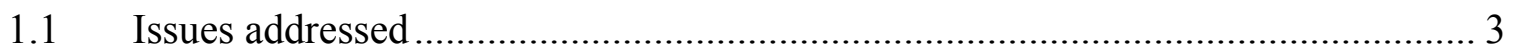

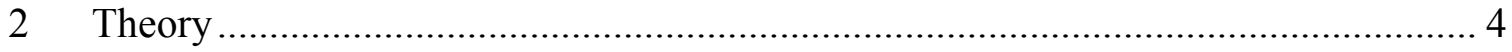

2.1 Fresnel's formulae and Parratt's recursive approach............................................ 5

2.2 Electron interaction with solids and $\mathrm{x}$-ray fluorescence..................................... 10

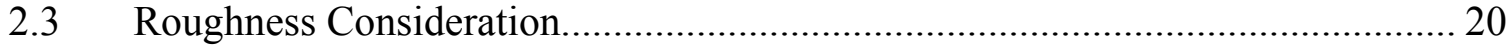

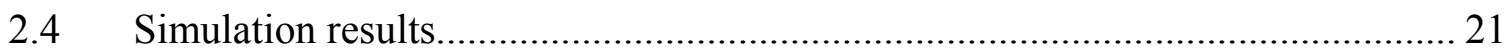

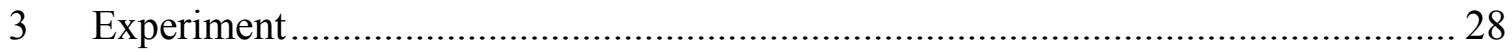

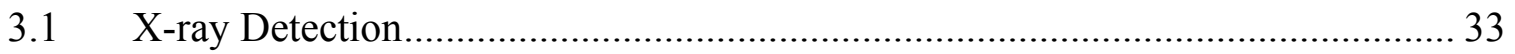

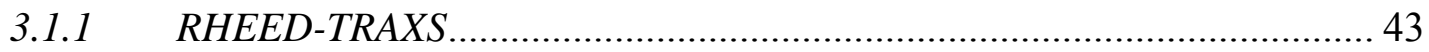

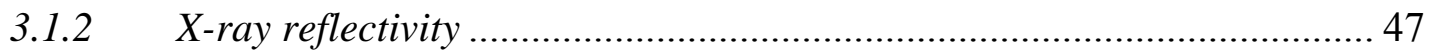

3.2 X-ray Photoelectron Spectroscopy (XPS) ………………………………….... 48

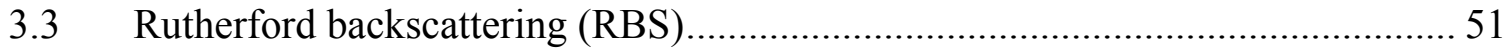

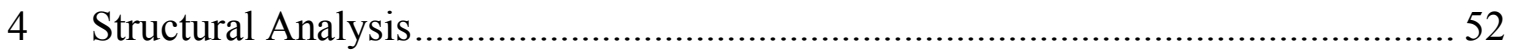

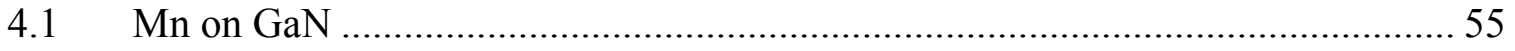

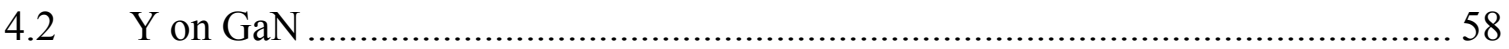

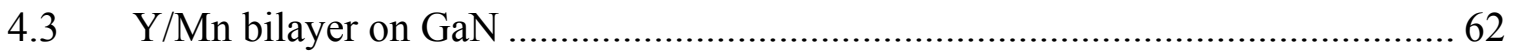

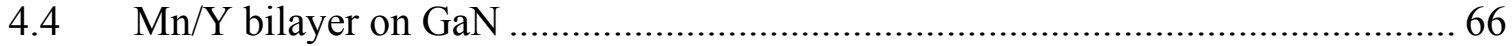

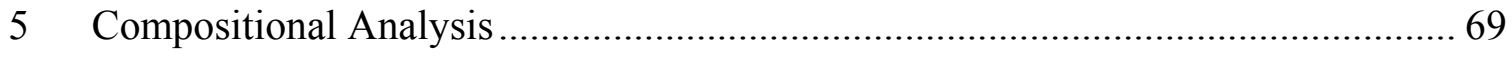

5.1 Angular dependence variation with refractive index ........................................ 70

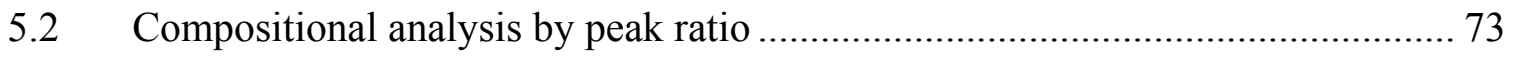

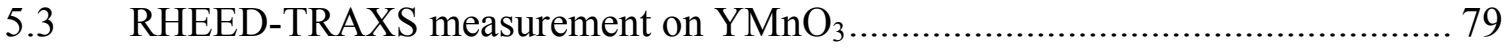


6 Conclusion and Future Directions . 


\section{Table of figures}

Figure 1 Reciprocity theorem. The plane waves are emanating from the detector and traveling towards the sample.

Figure 2 Plane wave incident upon N-stratified homogeneous media undergoes reflection and refraction at each boundary.

Figure 3 Bremsstrahlung production due to the deceleration of incident electrons on the Couloumbic field of the atoms in the solid. The electron with initial energy E0 can lose energy $\mathrm{E}=\mathrm{h} v$ and retain $\left(\mathrm{E}_{0}-\mathrm{E}\right)$ during its motion through the material. The energy loss due to bremsstrahlung radiation forms a continuous background in the energy dispersive spectrum.

Figure 4 (a) The interaction of incident electron with the inner shell electrons and the process of x-ray or auger emission. (b) Various excitation levels for the x-rays produced in process (a).

Figure 5 (a) The electron trajectories in $\mathrm{Y}$ on $\mathrm{Mn}$ on $\mathrm{GaN}$ sample obtained from CASINO for an electron beam angle of incidence of $2.7^{\circ}$ from sample surface and energy of $25 \mathrm{keV}$. (b)The $\mathrm{x}$-ray depth profile inside $\mathrm{Y}$ and Mn layers based on the trajectories in (a).

Figure 6 The simulation of angular dependence of $20 \mathrm{~nm}$ of $\mathrm{Y}, \mathrm{Ga}$ and $\mathrm{Mn}$ on a sapphire substrate. The position of the critical angle can be estimated from $\phi_{\text {critical }} \approx \sqrt{2 \delta_{2}}$ 23 Figure 7 The X-ray depth profiles for $50 \mathrm{~nm}$ thick layers of $\mathrm{Mn}(\mathrm{a})$ and $\mathrm{Y}$ (b) on $\mathrm{GaN}$ substrate. The best fit to the simulation data was obtained with the sum of two exponential functions.

Figure 8 Simulation results for Mn for various thickness values (a) and roughness values for $20 \mathrm{~nm}$ thick Mn layer (b). 26

Figure 9 Simulation results for $Y$ for various thickness values (a) and roughness values for $20 \mathrm{~nm}$ thick Y layer (b).

Figure 10 The oxide MBE system for growing $\mathrm{YMnO}_{3}$. (b) RHEED-TRAXS arm with $\mathrm{Si}$ $\mathrm{x}$-ray detector at the end of bellows arm.

Figure 11 The schematic of RHEED-TRAXS setup. Si detector and pre-amplifier are contained in a single unit that sits at the end of the RHEED-TRAXS arm. The data are collected on a computer by a multichannel analyzer (MCA).

Figure 12 XR100 x-ray detector and PX4 multichannel analyzer, (b) PX4 from the back and (c) the detector assembly 40 .

Figure 13 The intrinsic full energy detection efficiency for Si-PIN detectors. This efficiency corresponds to the probability that an X-ray will enter the front of the detector and deposit all of its energy inside the detector via the photoelectric effect.

(b) The probability of a photon undergoing any interaction in Si-PIN detectors, along with the probability of a photoelectric interaction which results in total energy deposition. The photoelectric effect is dominant at low energies but at higher energies above about $40 \mathrm{keV}$ the photons undergo Compton scattering 40. 36

Figure 14 (a) The block diagram of $x$-ray pulse processing using XR100 CR detector and PX4 pulse processor. (b) The signal output from various modules of the pulse processing circuitry. Preamplifier output is the output from preamplifier and input to analog prefilter; 
prefilter output is the output from analog prefilter; shaped output is the output from digital pulse processor 40 .

Figure 15 Signal processing through slow and fast channels to produce the spectrum 40 .

Figure 16 Throughput of the fast channel at various incoming count rates $40 \ldots \ldots \ldots \ldots . . . .41$

Figure 17 Input and output count rates with pile up rejection on and various peaking times 40 ....

Figure 18 The energy dispersive spectrum for $\mathrm{Mn}$ on $\mathrm{Y}$ (a) and $\mathrm{Y}$ on $\mathrm{Mn}$ (b) samples at angle of $20 \mathrm{mrad}$. Clearly visible are the $\mathrm{Mn}$ and $\mathrm{Ga} \mathrm{K} \alpha, \mathrm{K} \beta$ peaks and $\mathrm{Y} \mathrm{K} \alpha$ peak. The peak at $1.5 \mathrm{keV}$ in figure (b) is the $\mathrm{Al} \mathrm{K \alpha}$ peak from the sapphire template. 45 Figure 19 The sectioned-off portion of EDS showing the characteristic peak and the best fit using a Gaussian function and a linear background for (a) Mn, (b) Y and (c) GaN. (d) shows the variation in $\mathrm{Ga} \mathrm{K \alpha}$ intensity at various take-off angles. 46

Figure 20 The estimation of the binding energy of an electron using monochromatic radiation and the kinetic energy based filtering of the ejected electrons in XPS experiment. XPS can be used for quantitative compositional analysis and determining the oxidation states of the elements.

Figure 21 (a) The area under Mn Ka peak at the grazing angle of 9 mrad. The linear trend for small thickness flattens out as the thickness is increased, signifying that the contribution from deeper layers has diminishing effect on the intensity. No collimating slit was used. 1 monolayer of $\mathrm{Mn}$ is 2.3 angstrom thick. (b)The angular dependence of $\mathrm{Mn} \mathrm{K} \alpha$ peaks for varying thickness during initial experiments. The thickness estimate is based on crystal monitor calibration of Mn flux. No collimating slit was used. 53 Figure 22 (a) RHEED-TRAXS measurements and (b) XRR measurements on a Mn film sample. The symbols are the data and the solid curves are the non-linear fits to the data. (i) and (ii) correspond to data obtained prior and after the Al deposition, respectively. The parameters used in this fit are shown in Table 1. (c) The angular dependence of Ga from $\mathrm{GaN}$ substrate that was used to calibrate the angle.

Figure 23 (a) RHEED-TRAXS measurements and (b) XRR measurements on a Y film sample. The symbols are the data and the solid curves are the non-linear fits to the data. (i) and (ii) correspond to data obtained prior and after the Al deposition, respectively. The parameters used in this fit are shown in Table 1. (c) The angular dependence of Ga from $\mathrm{GaN}$ substrate that was used to calibrate the angle.

Figure 24 RHEED-TRAXS and measurements using (a) Mn K $\alpha$ fluorescence and (b) Y $\mathrm{K}$ fluorescence on the GaN/Mn/Y/Al sample. In (b), (i), (ii) and (iii) are data obtained after deposition of the Mn layer, the Y layer and the Al layer, respectively. Symbols represent the data, solid curves non-linear fits to the data. In (b), (i) and (ii) are data obtained after the deposition of Y layer and the Al layer. The parameters used for the fits are shown in Table 3. (c) X-ray reflectivity measurements. (d) Ga angular dependence for angular calibration.

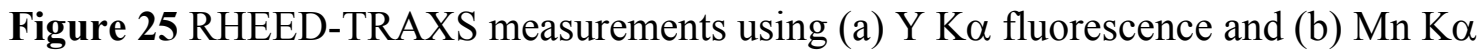
fluorescence on the GaN/Y/Mn/Al sample. In (a), (i) and (ii) are data obtained after deposition of the Y layer and the Mn layer, respectively. In (b), (i) and (ii) are data obtained after deposition of the Mn layer and the Al layer. Symbols represent the data, solid curves non-linear fits to the data. The parameters used for the fits are shown in 
Table 4. (c) X-ray reflectivity measurements. (d) Ga angular dependence for angular

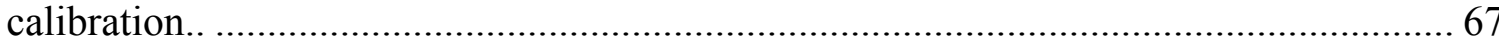

Figure 26 The variation in the angular dependence of $\mathrm{Mn}$ and $\mathrm{Y}$ fluorescence coming off $20 \mathrm{~nm} \mathrm{YMnO}_{3}$ layer based on the variation in slight change in the refractive index....... 72 Figure 27 XRR fitting and thickness estimates for Y/Mn bilayer samples used for estimating relative sensitivity of $\mathrm{Mn}$ and $\mathrm{Y}$.

Figure 28 RHEED-TRAXS angular dependence for two Y/Mn bilayer samples. (a) and (b) are $\mathrm{Mn}$ and $\mathrm{Y}$ angular dependences for $13.1 \pm 0.5 \mathrm{~nm}$ thick $\mathrm{Mn}$ and $17.0 \pm 0.7 \mathrm{~nm}$ thick $Y$ in first bilayer sample. Peak areas greater at angles greater than $26 \mathrm{mrad}$ were used to calculate the ratio in Equation 50. (c) and (d) are Mn and Y angular dependences for $15.1 \pm 0.5 \mathrm{~nm}$ thick $\mathrm{Mn}$ and $18.7 \pm 0.6 \mathrm{~nm}$ thick $\mathrm{Y}$ in the second bilayer sample. Peak areas greater at angles greater than $21 \mathrm{mrad}$ were used to calculate the ratio in Equation 50 .

Figure 29 The thickness dependence of $\mathrm{Y}$ to $\mathrm{Mn} \mathrm{K} \alpha$ peak ratios at various angles in $\mathrm{YMnO}_{3}$ samples. The data points are reached upon by scaling the experimental peak ratios by the sensitivity factor as described in Equation 52 .

Figure $30(a-c)$ XRR scans and best fits, (d-e) RHEED-TRAXS scans and simulation based on the thickness values obtained by XRR for the 3 samples analyzed using RBS and XPS measurements.

Figure 31 The XPS survey scan (a) and the detailed scans (b- d) of $54.5 \mathrm{~nm}$ thick $\mathrm{YMnO}_{3}$ sample used to determine the relative compositions of $\mathrm{Y}, \mathrm{Mn}$ and $\mathrm{O}$. The survey scan shows both photoelectric electrons and Auger electrons peaks. The peaks labeled as KLL, MNN and LMM are the Auger peaks.

Figure $32 \mathrm{RBS}$ results for $54.5 \mathrm{~nm}$ thick $\mathrm{YMnO}_{3}$ sample. (a) The yield of backscattered $\mathrm{He}++$ ions of $2.275 \mathrm{MeV}$ energy. The detector angle is $108^{\circ}$. The scaled intensities of various peaks give their relative compositions. (b) The relative compositions of $\mathrm{Y}, \mathrm{Mn}, \mathrm{O}$, $\mathrm{Ga}$ and $\mathrm{N}$ with depth. The given uncertainty in the estimates of $\mathrm{Y}, \mathrm{Mn}$ and $\mathrm{O}$ compositions is $\pm 0.5, \pm 1$ and \pm 3 respectively.

Figure 33 (a) shows the average of unscaled Y/Mn peak area ratios at angles greater than $16 \mathrm{mrad}$ for 3 samples. The solid lines are the linear fits. Open triangle at $(1,0.37)$ is based on relative sensitivity from bilayer samples, inverted triangle at $(1,0.34)$ is the relative sensitivity factor corrected for refractive indices for $\mathrm{Mn}$ and $\mathrm{Y}$ energies. Open circle is the stoichiometric point $(1,0.29 \pm 0.02)$ based on the linear fit to data point. (b) A linear fit to the RHEED-TRAXS and RBS results as shown in Table 5. The open circle at $(1,0.87 \pm 0.04)$, based on the linear fit, shows that RHEED-TRAXS underestimated the compositional ratio because of the ignored secondary effects. 


\section{List of tables}

Table 1 Structural parameters for the GaN/Mn/Al sample obtained from x-ray reflectivity (XRR) and RHEED-TRAXS. The thickness of the layer is $d$ and the interfacial roughness is $\sigma$. The uncertainties in the values were obtained from the non-linear least squares fit procedure. The $\mathrm{Mn} \_\mathrm{GaN}$ data were obtained after the Mn deposition and the Al_Mn_GaN profile was obtained after the Al layer deposition. The characteristic x-ray peak used for the data analysis is indicated

Table 2 Structural parameters for the $\mathrm{GaN} / \mathrm{Y} / \mathrm{Al}$ sample obtained from $\mathrm{x}$-ray reflectivity (XRR) and RHEED-TRAXS. The thickness of the layer is $d$ and the interfacial roughness is $\sigma$. The uncertainties in the values were obtained from the non-linear least squares fit procedure. The $\mathrm{Y}_{-} \mathrm{GaN}$ data were obtained after the $\mathrm{Y}$ deposition and the Al_Y_GaN profile was obtained after the Al layer deposition. The characteristic $\mathrm{x}$-ray peak used for the data analysis is indicated.

Table 3 Structural parameters for the GaN/Mn/Y/Al sample obtained from X-ray reflectivity (XRR) and RHEED-TRAXS. The thickness of the layer is $d$ and the interfacial roughness is $\sigma$. The uncertainties in the values were obtained from the nonlinear least squares fit procedure. The Mn_GaN, Y_Mn_GaN, Al_Y_Mn_GaN data were obtained after the Mn, Y, and Al depositions, respectively. The characteristic X-ray peak used for the data analysis is indicated 65

Table 4 Structural parameters for the GaN/Y/Mn/Al sample obtained from X-ray reflectivity (XRR) and RHEED-TRAXS. The thickness of the layer is $d$ and the interfacial roughness is $\sigma$. The uncertainties in the values were obtained from the nonlinear least squares fit procedure. The Y_GaN, Mn_Y_GaN, Al_Mn_Y_GaN data were obtained after the $\mathrm{Y}, \mathrm{Mn}$, and $\mathrm{Al}$ depositions, respectively. The characteristic x-ray peak used for the data analysis is indicated. Y signal from under $\mathrm{Al}$ and $\mathrm{Mn}$ layers was too weak to be analyzed

Table 5 The comparative table for relative composition of $\mathrm{YMnO}_{3}$ samples. XPS results are off by a constant factor because of the sensitivity factor used and showed lower sensitivity than RBS and RHEED-TRAXS results. RHEED-TRAXS ratios are derived from the average of $\mathrm{Y}$ to $\mathrm{Mn}$ peak ratios at angles greater than $16 \mathrm{mrad}$, scaled by the sensitivity factor of $2.71 \pm 0.08$ for $\mathrm{Mn}$ to $\mathrm{Y}$ response derived in section 5.2 and correction factor of 1.1 for $\mathrm{Mn}$ to $\mathrm{Y}$, to account for the difference in refractive index as shown in Figure 30. 


\section{Introduction}

The potential use of x-rays for qualitative and quantitative analysis of elements and thin films was appreciated soon after their discovery. The reflectivity of x-rays from a thin layer of nickel on glass substrate was shown to exhibit fringes dependent on the film thickness, in the early twentieth century [1]. In 1954, L. G. Parratt worked out a detailed method for the analysis of layered materials using x-ray reflectivity [2] .

X-rays emitted from ionized atoms have energies characteristic of the element involved and intensity proportional to both the elemental concentration and the strength of the exciting source. This provides a powerful tool for elemental analysis. Yoneda and Horiuchi found in 1971 that x-ray fluorescence (XRF) was a sensitive tool for microanalysis if an optically flat surface is used as a substrate and grazing x-rays are used to probe the surface [3]. The sensitivity was enhanced because of the evanescent nature of x-rays near the critical angle of total reflection. This limited the penetration of x-rays to the first few nanometers, significantly increasing the detection limit of the analyte on top. This essentially marked the beginning of XRF spectroscopy.

Later, Becker et. al. [4]demonstrated the presence of evanescent x-rays by detecting their absorption and excitation near the target surface. They also studied their angular dependence and established the principle of microscopic reversibility for incident and grazing x-rays. Since then, angle dependent XRF has become a widely accepted tool for quantitative analysis of impurities, adsorbed molecules and layers on substrates.

Barbee T. W. et. al. studied synthetic multilayered structures using angular dependent total x-ray reflection (AD-TRX) for the first time in 1984 and found good 
agreement between calculations and experiment in both evanescent and standing wave regimes [5].

Hasegawa et al first reported the in-situ x-ray fluorescence analysis using an electron gun for reflection high energy electron diffraction (RHEED) as the excitation source and the technique was referred to as RHEED-total-refection-angle x-ray spectroscopy (RHEED-TRAXS) [6]. An elemental sensitivity of 0.01 monolayers was reported. Since then, the use of RHEED-TRAXS analysis of the sample surface during epitaxial growth has been reported by various groups [7-12]. The depth distribution of surface elements was studied by varying the incident angle of the electron beam to excite $\mathrm{x}$-rays from various depths and the glancing angle of x-rays. Various growth modes of the elements such as substitution, alloying and floating could be studied.

Tsuji et. al. used the grazing angle x-ray fluorescence to study single and multilayered structures using both an x-ray source and electron gun for excitation [13-16]. Tsuji and Tctsuoka also utilized an electron beam at normal incidence to investigate the angular dependence of $\mathrm{x}$-ray fluorescence at grazing exit angle (AD-TRX) and found a good agreement between the theory and the experimental data [13]. Grazing angle fluorescence has also been studied using various excitation conditions, including synchrotron radiation [17-19] and in conjugation with x-ray reflectivity [20].

Here, RHEED-TRAXS is described as an absolute method for thin film analysis by utilizing principles similar to the analysis of a multilayered structure by AD-TRX. The angular dependence of the energy dispersive spectrum of the fluorescence radiation has been shown to yield information about the layered structure because the critical angle and the shape of the angular dependence are dependent upon the film thickness and on 
properties of overlying and underlying layers. Thus, simultaneous determination of film thickness and interfacial roughness values was possible. The determination of relative compositions of the constituent elements without the use of standards was investigated. The calibration technique proposed was not met with success and suggested the need of a standard sample of known thickness.

Simultaneous, in-situ structural and chemical characterization should be a very powerful tool that can be useful for a wide variety of thin film growth techniques, particularly for materials where small changes in stoichiometry can result in large changes in physical properties, such as complex oxides. Since a RHEED electron beam gun is a common feature on MBE systems, the addition of a relatively inexpensive x-ray detector on a translation stage adds the capability of exhaustive in-situ analysis where growth parameters can be studied and controlled to a greater degree.

\subsection{Issues addressed}

The initial aim of the initiative was to come up with a technique for flux calibration that has better sensitivity than quartz crystal monitor (QCM) currently used for flux calibration for the MBE growth of the ferroelectric oxide $\mathrm{YMnO}_{3}$. The feasibility and sensitivity of the technique was explored and its viability as a standard technique for structural and compositional analysis will be discussed. 


\section{Theory}

The section describes the theoretical treatment for the data analysis based on Parratt's formalism and the simulation of electrons' trajectories in the solid. The expression for electric field inside a multilayered structure has been worked out explicitly (Equation 24). Also, no precedence was found for the incorporation of $x$-ray profile due to RHEED gun in the grazing exit fluorescence that resulted in Equation 35 and the application of the formalism in non-linear fitting routine to obtain quantitative results.

According to the reciprocity theorem, a point source at $r$ will produce at $r_{0}$ the same electric field as a point source at $r_{0}$ will produce at $r$ [22] (Figure 1). As a result, the fluorescence intensity at grazing exit of $x$-rays can be calculated using the same approach to calculate the electric field due to grazing incidence of x-rays. Becker et al have demonstrated this principle of microscopic reversibility by experimentally establishing the equivalence of the grazing incidence and grazing exit angles for XRF intensity [4].

Reversing the position of the source of fluorescence with the detector, the part of XRF intensity from scatterers that is detected at the detector position can be decomposed into the plane waves emanating from the detector position, within the framework of the distorted wave approximation (DWA)[23].

By expressing the electric field inside a medium as the sum of incident plane wave and reflected plane waves from the interface, the field intensity at any point inside the medium can be calculated (Figure 1). The reflectance and transmittance at various interfaces for multiple layers can be calculated recursively, keeping in mind that the reflectance from the bottom of the substrate is zero [2] . 


\subsection{Fresnel's formulae and Parratt's recursive approach}

Consider a plane wave incident upon a thin multilayered structure over a substrate (Figure 2). The expressions for the electric fields of incident, reflected and transmitted beams at a perpendicular distance $\mathrm{z}$ from the first interface can be written

$$
\begin{aligned}
& E_{1}\left(z_{1}\right)=E_{1}(0) \exp \left\{i\left[\omega t-\left(k_{1, x} x_{1}+k_{1, z} z_{1}\right)\right]\right\} \\
& E_{1}^{R}\left(z_{1}\right)=E_{1}^{R}(0) \exp \left\{i\left[\omega t-\left(k_{1, x} x_{1}-k_{1, z} z_{1}\right)\right]\right\} \\
& E_{2}\left(z_{2}\right)=E_{2}(0) \exp \left\{i\left[\omega t-\left(k_{2, x} x_{2}+k_{2, z} z_{2}\right)\right]\right\} \\
& E_{2}^{R}\left(z_{2}\right)=E_{2}^{R}(0) \exp \left\{i\left[\omega t-\left(k_{2, x} x_{2}+k_{2, z} z_{2}\right)\right]\right\}
\end{aligned}
$$

$k_{x}$ and $k_{z}$ are the $\mathrm{x}$ and $\mathrm{z}$ components of the wave vector and subscripts 1 and 2 refer to the respective media. $k_{1}$ and $k_{2}$ are the propagation vectors in vacuum $\left(\frac{2 \pi}{\lambda_{1}}\right)$ and in the topmost film $\left(\frac{2 \pi}{\lambda_{2}}\right)$, respectively. So using Snell's law, $n_{1}^{2} k_{2}^{2}=n_{2}^{2} k_{1}^{2}$ with $n_{1} \approx 1$ and $n_{2}=1-\delta_{2}-i \beta_{2}$, the refractive index of medium 2 . The real term $\delta$ is related with the dispersion and the imaginary term $\beta$ with absorption of the $x$-rays. In terms of the $x$ - and z-component of $k_{2}$

$$
\begin{aligned}
& k_{2}^{2}=\frac{n_{2}^{2} \cdot k_{1, x}^{2}}{\cos ^{2} \phi_{1}} \approx\left(1-2 \delta_{2}-2 i \beta_{2}+\phi_{1}^{2}\right) k_{1, x}^{2}, \\
& k_{2, x}^{2}+k_{2, z}^{2}=\left(1-2 \delta_{2}-2 i \beta_{2}+\phi_{1}^{2}\right) \cdot k_{1, x}^{2} .
\end{aligned}
$$

Since the angle of incidence is very small, $k_{2, x} \approx k_{1 . x} \approx k_{1}$, so that

$$
k_{2 . z} \approx\left(\phi_{1}^{2}-2 \delta_{2}-2 i \beta_{2}\right)^{1 / 2} k_{1}=f_{2} k_{1} .
$$




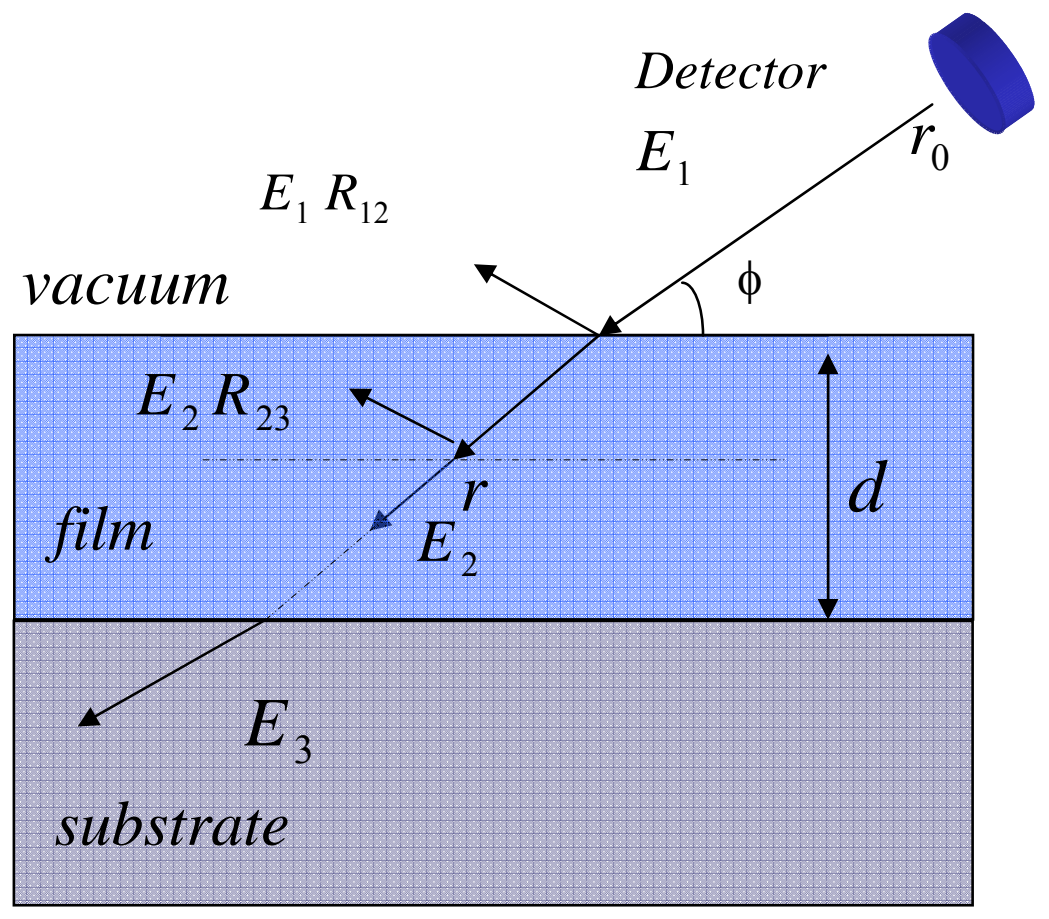

\section{Figure 1}

Reciprocity theorem. The plane waves are emanating from the detector and traveling towards the sample.

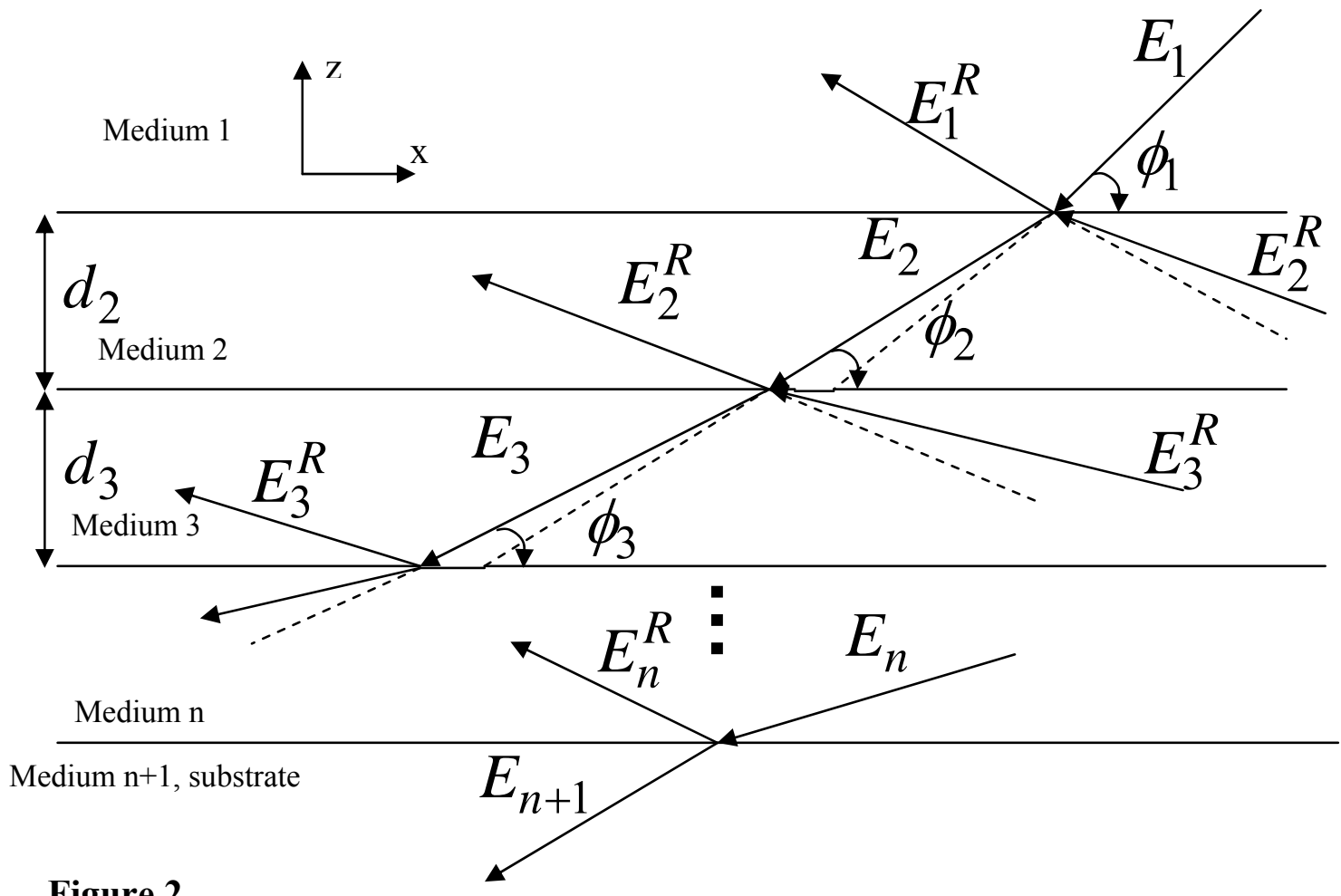

Figure 2

Plane wave incident upon N-stratified homogeneous media undergoes reflection and refraction at each boundary. 
The Fresnel reflectance coefficient for the tangential component of the electric field incident on and reflected from the interface between medium 1 and 2 is,

$$
F_{1,2}=\frac{E_{1}^{R}}{E_{1}}=\frac{n_{1} \sin \phi_{1}-n_{2} \sin \phi_{2}}{n_{1} \sin \phi_{1}+n_{2} \sin \phi_{2}}
$$

where $\mathrm{n}_{1}$ is the refractive index of medium 1, i.e., air and $\sin \phi_{2}=\frac{\left|k_{2, z}\right|}{\left|k_{2}\right|} \approx \frac{\left|k_{2, z}\right|}{\left|k_{1}\right|}=f_{2}$. Therefore,

$$
F_{1,2} \approx \frac{\phi_{1}-f_{2}}{\phi_{2}+f_{2}}=\frac{f_{1}-f_{2}}{f_{1}+f_{2}}
$$

where $f_{1}=\left(\phi_{1}^{2}-2 \delta_{1}-2 i \beta_{1}\right)^{1 / 2}=\phi_{1}$, since $\delta_{1}$ and $\beta_{1}$ are zero for vacuum.

Since the tangential component of the electric field and its first derivative should be continuous across an interface, at interface 1 i.e., the air-film interface,

$$
\begin{aligned}
& \left.E_{1}^{\text {Total }}\right|_{\text {boundary }}=\left.E_{2}^{\text {Total }}\right|_{\text {boundary }} \\
& \left.\frac{\partial E_{1}^{\text {Total }}}{\partial z}\right|_{\text {boundary }}=\left.\frac{\partial E_{2}^{\text {Total }}}{\partial z}\right|_{\text {boundary }}
\end{aligned}
$$

Using Equations 1 to 4 and realizing that $z=0$ at the boundary, the following expressions can be worked out from Equations 10 and 11.

$$
\begin{aligned}
& E_{1}+E_{1}^{R}=E_{2}+E_{2}^{R}, \\
& k_{1, z}\left(E_{1}-E_{1}^{R}\right)=k_{2, z}\left(E_{2}-E_{2}^{R}\right),
\end{aligned}
$$

But $k_{1, z}=k_{1} \sin \phi_{1} \approx k_{1} \phi_{1}=k_{1} f_{1}$ and $k_{2, z}=f_{2} k_{1}$ from Eq. 7, so that

$$
\left(E_{1}-E_{1}^{R}\right)=\left(E_{2}-E_{2}^{R}\right) \frac{f_{2}}{f_{1}}
$$

and Eqs. from (12) and (14), 


$$
\frac{E_{1}^{R}}{E_{1}}=\frac{\left(f_{1}-f_{2}\right)+\frac{E_{2}^{R}}{E_{2}}\left(f_{1}+f_{2}\right)}{\left(f_{1}+f_{2}\right)+\frac{E_{2}^{R}}{E_{2}}\left(f_{1}-f_{2}\right)}=\frac{F_{1,2}+\frac{E_{2}^{R}}{E_{2}}}{1+\frac{E_{2}^{R}}{E_{2}} F_{1,2}} .
$$

For the interface between medium 2 and 3, equations analogous to Eqs. 1 to 4 can be written using the same continuity conditions as before. At the interface between films 2 and 3 ,

$$
\begin{aligned}
& a_{2} E_{2}+a_{2}^{-1} E_{2}=E_{3}+E_{3}^{R}, \\
& \left(a_{2} E_{2}-a_{2}^{-1} E_{2}\right)=\left(E_{3}-E_{3}^{R}\right) \frac{k_{3, z} k_{1}}{k_{2, z} k_{1}}=\left(E_{3}-E_{3}^{R}\right) \frac{f_{3}}{f_{2}},
\end{aligned}
$$

where

$$
a_{2}=\exp \left(-i k_{2, z} d_{2}\right)=\exp \left(-i k_{1} f_{2} d_{2}\right)
$$

Combining Eqs. (16) and (17) leads to

$$
a_{2}^{-2} \frac{E_{2}^{R}}{E_{2}}=\frac{\left(f_{2}-f_{3}\right)+\frac{E_{3}^{R}}{E_{3}}\left(f_{2}+f_{3}\right)}{\left(f_{2}+f_{3}\right)+\frac{E_{3}^{R}}{E_{3}}\left(f_{2}-f_{3}\right)}=\frac{F_{2,3}+\frac{E_{3}^{R}}{E_{3}}}{1+\frac{E_{3}^{R}}{E_{3}} F_{2,3}} .
$$

Hence, the reflectance for the $n$th layer can be expressed in terms of the reflectance of $(n+1)$ th layer. If $(n+1)$ th layer is the substrate itself, then $\frac{E_{n+1}^{R}}{E_{n+1}}=0$, so that

$$
a_{n}^{-2} \frac{E_{n}^{R}}{E_{n}}=\frac{f_{n}-f_{n+1}}{f_{n}+f_{n+1}} .
$$

Now the reflectance from all the boundaries can be written down in terms of the incident intensity $E_{1}$ by back-substituting $\frac{E_{m}^{R}}{E_{m}}$ starting from the bottom layer. The above mentioned procedure can be summed up by the following recursive formulae. 
Defining

$$
\begin{aligned}
& R_{n, n+1}=\frac{E_{n}^{R}}{E_{n}}, \\
& R_{n-1, n}=a_{n-1}^{2}\left[\frac{R_{n, n+1}+F_{n-1, n}}{R_{n, n+1} \cdot F_{n-1, n}+1}\right],
\end{aligned}
$$

where

$$
F_{n-1, n}=\frac{f_{n-1}-f_{n}}{f_{n-1}+f_{n}}
$$

assuming $a_{1}=1$ for generalized treatment.

This constitutes Parratt's recursive approach and enables to express the electric field inside any layer of a multilayered sample. Following this approach, the electric field amplitude transmitted into $n$th layer and reflected back from the $(n, n+1)$ interface can be written as

$$
E_{n}=\frac{\left(a_{1}+a_{1}^{-1} R_{1,2}\right)\left(a_{2}+a_{2}^{-1} R_{2,3}\right) \ldots\left(a_{n-1}+a_{n-1}^{-1} R_{n-1, n}\right)}{\left(1+R_{2,3}\right)\left(1+R_{3,4}\right) \ldots\left(1+R_{n, n+1}\right)} E_{1}
$$

and

$$
E_{n}^{R}=R_{n, n+1} E_{n}
$$

The total electric field at any point $\mathrm{z}$ inside the $n$th layer be can written as

$$
E_{n}^{\text {Total }}(z, \phi)=E_{n} \exp \left(-i \frac{2 \pi z f_{n}}{\lambda}\right)+E_{n}^{R} \exp \left(i \frac{2 \pi z f_{n}}{\lambda}\right),
$$

where $f_{n}=\left(\phi^{2}-2 \delta_{n}-2 i \beta_{n}\right)^{1 / 2}$.

Hence, the total intensity $I(\phi)$ at grazing angle $\phi$, coming from all the scatterers along the entire depth of the layer can be obtained by integrating the intensity $\left|E_{n}^{\text {Total }}(z, \phi)\right|^{2}$, so that 


$$
I(\phi) \propto \int_{0}^{d_{n}}\left|E_{n}^{\text {Total }}(z, \phi)\right|^{2} d z
$$

The above expression holds for a homogeneous distribution of x-ray sources along the entire depth. But for the x-rays produced by the electrons grazing the surface of the sample, as in our case, $\mathrm{x}$-ray production is not uniform and the depth dependence of the $\mathrm{X}$-ray production must be taken into account.

\subsection{Electron interaction with solids and $x$-ray fluorescence}

The electrons interaction inside the sample can be divided into two classes:

1. Elastic scattering events that affect the trajectory of the electrons without affecting their kinetic energy. This process is responsible for the phenomenon of electron backscattering.

2. Inelastic scattering events that transfer energy to the specimen and leads to the generation of secondary electrons, auger electrons, characteristic x-rays and bremsstrahlung x-rays. It also leads to cathodoluminescence and lattice vibrations (phonons).

The inelastic interactions of electrons can yield valuable spectroscopic information about the material as the energy lost depends on properties like ionization energy, chemical bonding and dielectric constant of the medium. In the order of increasing energy loss, the main processes under this category can be summarized as follows [24, 25]:

1. Phonon excitation: Electrons hitting the sample deposit a substantial amount of energy by small energy loss events $(\leq 1 \mathrm{eV})$ to cause excitation of the lattice oscillations. For thin samples $(\leq 100 \mathrm{~nm})$ or high beam currents $(\geq 1 \mu \mathrm{A})$ the 
temperature rise of several hundred degrees Celsius is possible in nonconducting bulk samples.

2. Plasmon excitation: In metals, a flux of electrons can cause fluctuations in the 'sea' of electrons that are regular (plasmons) due to the periodicity in the crystal structure. Excitation of plasmons involves energy of about $15 \mathrm{eV}$.

3. Secondary electron excitation: In semiconductors or insulators, incoming electrons can eject loosely bound electrons from their shells with sufficient kinetic energy for subsequent motion through the solid. These electrons undergo the same processes as primary ones and may have sufficient energy to overcome the surface barrier and escape the sample as secondary electrons. A majority of ejected electrons have energies $\leq 10 \mathrm{eV}$.

4. Bremsstrahlung or continuum x-ray generation: The electrons experience a decelerating force as soon as they enter the sample because of the Coulombic field of the atoms. The decelerating electrons emit electromagnetic radiation known as bremsstrahlung or braking radiation. The energy loss can be from zero to the beam energy forming a continuous spectrum (Figure 3). Though the bremsstrahlung is dependent upon the direction of the flight of the beam of electrons relative to the scattering center, the effect of the elastic scattering is to randomize the trajectories, so that the effective bremsstrahlung generation, as seen by the observer, is nearly isotropic.

5. Characteristic x-rays: A sufficiently energetic electron can cause the ejection of an inner shell electron and leave an atom in an excited state. An electron from an outer shell falls in its place and the excess energy is released by emitting an x-ray 
or an auger electron (Figure 4(a)). X-ray generation and Auger electron production are competing processes in atomic relaxation. As the energy of a shell and sub-shell is sharply defined, the emitted x-ray photon contains very specific information about the atom involved. Figure 4(b) shows various allowed transitions in an atom. The minimum energy required to eject an $\mathrm{x}$-ray also has a well-defined value called the 'critical ionization energy'. For Y and Mn, the characteristic L emissions at $2.00 \mathrm{keV}$ and $0.65 \mathrm{keV}$, respectively, were too low to be detected efficiently above the noise level. Hence, $\mathrm{K}$ emissions at around 15 $\mathrm{keV}$ and $6 \mathrm{keV}$ were used for the statistical analysis. The partitioning of deexcitation process between x-ray and Auger electron emission can be described as:

$$
\omega+a=1
$$

$\omega$ is the fluorescent yield and a is the auger yield. For elements with low atomic number Auger emission is the preferred mechanism. The probability of x-ray emission goes up with increase in atomic number. The fluorescent yield can be described as

$$
\omega=\frac{Z^{4}}{A+Z^{4}}
$$

for an element with atomic number $Z$. A is about $9 \times 10^{5}$ for K-series emission [26]. With the above relation, Y and Mn have the fluorescence yield of 0.72 and 0.30 , respectively.

The processes of elastic and inelastic scattering occur concurrently. Elastic scattering causes the beam electrons to deviate from their original trajectory and diffuse into the solid and inelastic scattering reduces the energy until it losses all its energy or is 
ejected as a backscattered electron. The electron interacting with an atom undergoes inelastic or elastic scattering through a random process. 


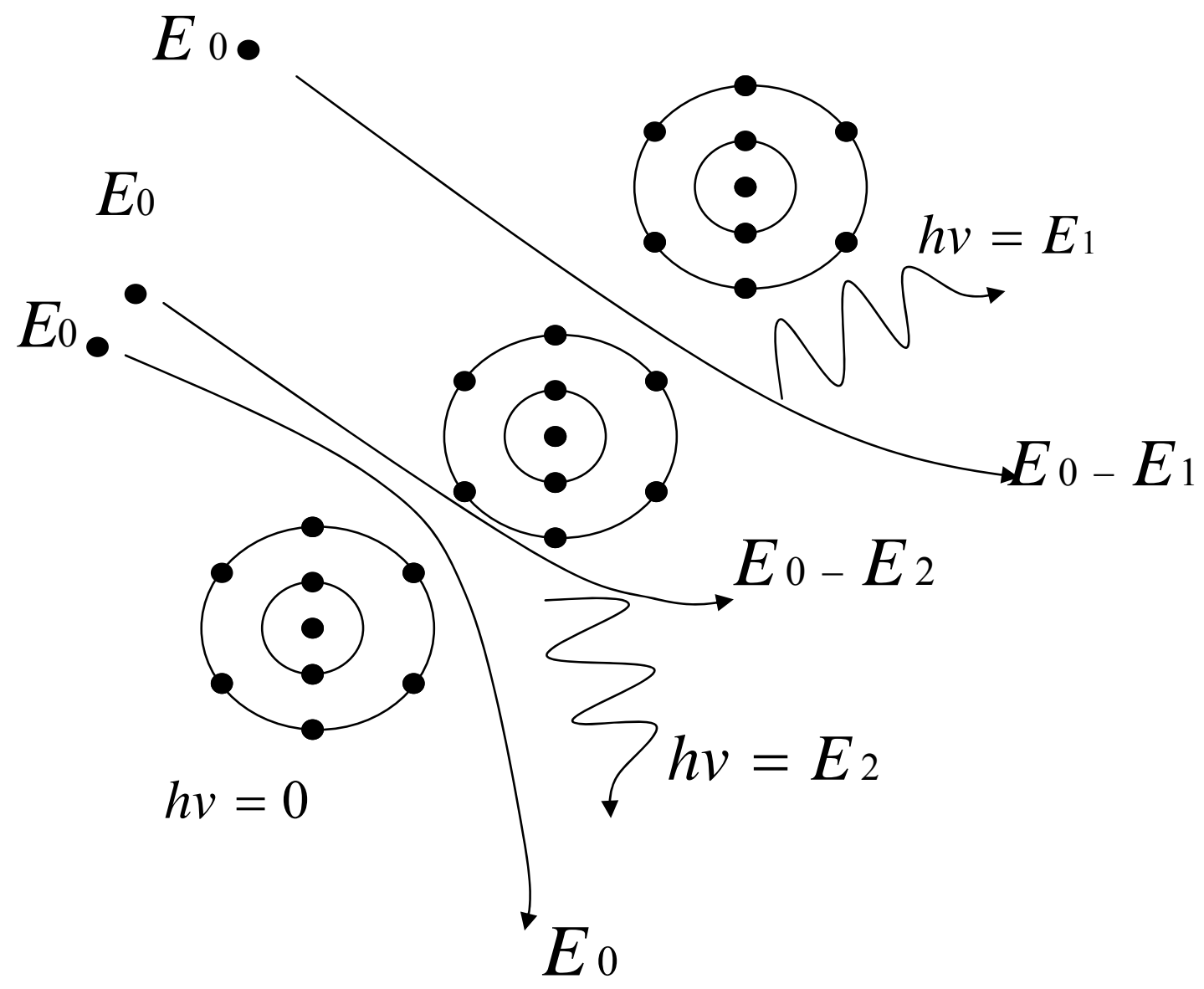

Figure 3

Bremsstrahlung production due to the deceleration of incident electrons on the Couloumbic field of the atoms in the solid. The electron with initial energy $\mathrm{E}_{0}$ can lose energy $\mathrm{E}=\mathrm{h} v$ and retain $\left(\mathrm{E}_{0}-\mathrm{E}\right)$ during its motion through the material. The energy loss due to bremsstrahlung radiation forms a continuous background in the energy dispersive spectrum. 
(a)
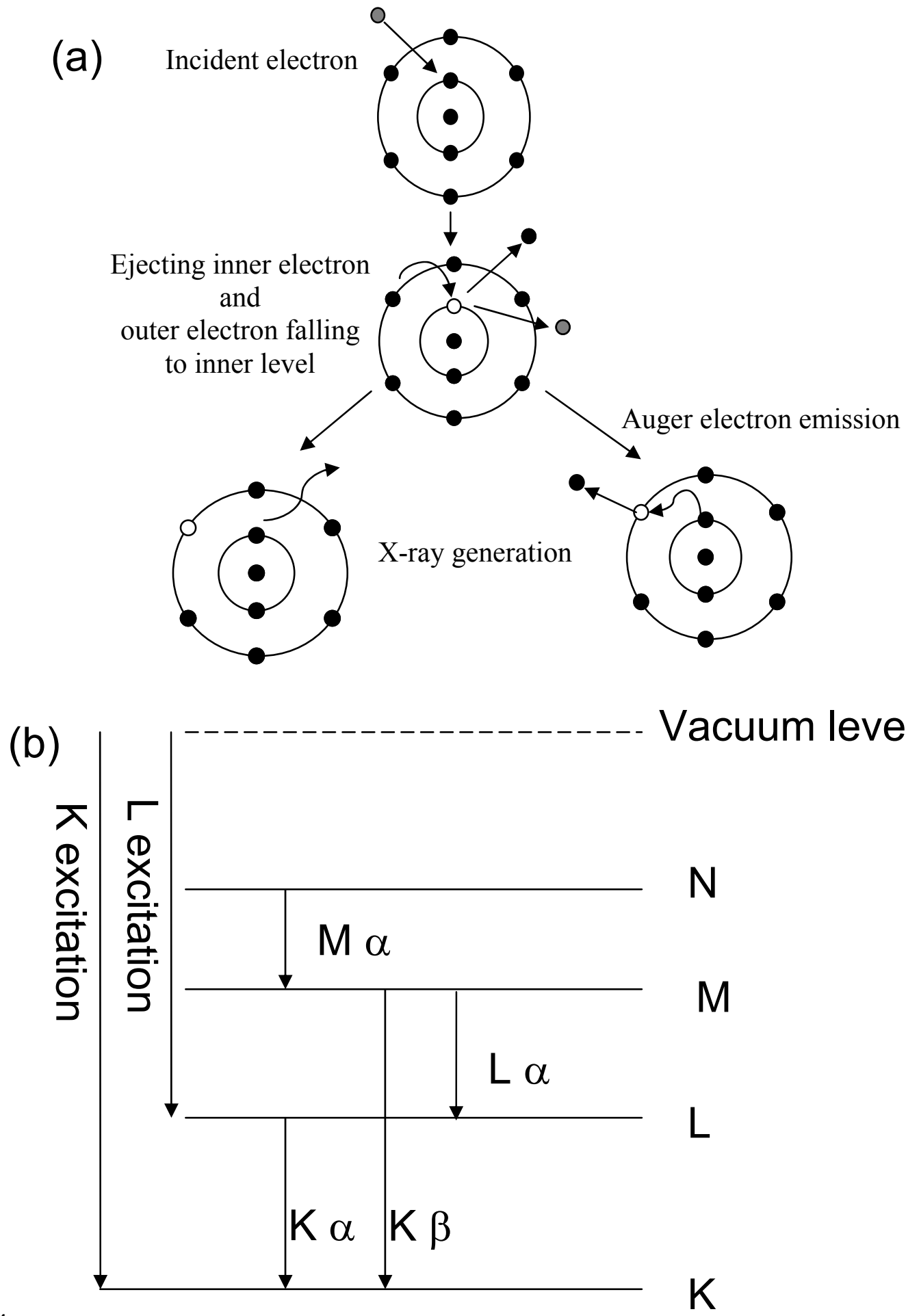

Figure 4

(a) The interaction of incident electron with the inner shell electrons and the process of $x$ ray or auger emission. (b) Various excitation levels for the x-rays produced in process (a). 


\section{Monte Carlo simulation of electron trajectories}

Monte Carlo simulation of electron trajectories is a powerful way to visualize the scattering process inside the sample and generate useful information about energy loss, backscattering and x-ray generation, among other things. A popular free software called CASINO (monte Carlo SImulation of electroN trajectories in sOlids) was used for the simulation. The basic steps for constructing the simulation are as follows [27]:

The initial penetration angle for the beam is set by the user and no scattering angle is calculated. The actual landing position of an electron is calculated using a random number. The distance between two collisions (L) is calculated using the mean free path of the electron undergoing elastic scattering inside the material multiplied by another random number as a weight factor to generate an appropriate distribution.

$$
L=-\lambda_{e l} \log (R)
$$

where

$$
\frac{1}{\lambda_{e l}}=\rho N_{0} \sum_{i=1}^{n} \frac{C_{i} \sigma_{e l}^{i}}{A_{i}}
$$

where $L=$ distance between scattering, $R=$ random number, $\lambda_{e l}=$ mean free path, $\rho=$ density of the region, $\mathrm{N}_{0}=$ Avogadro's number , $\sigma_{e l}=$ total cross-section determined using tabulated values. $C_{i}$ and $A_{i}$ are the weight fraction and the atomic weight of the element.

The program neglects the effect of the inelastic scattering and groups all the energy losses into a continuous function. This is called the continuous energy loss approximation. With this assumption,

$$
E_{i+1}=E_{i}+\frac{d E}{d S} L
$$




$$
\frac{d E}{d S}=\frac{-7.85 \times 10^{-3} \rho}{E_{i}} \cdot \sum_{j=1}^{n} \frac{C_{j} Z_{j}}{A_{j}} \ln \left(1.116\left(\frac{E_{i}}{J_{j}}+k_{j}\right)\right)[\mathrm{keV} / \mathrm{nm}]
$$

where $Z_{j}$ and $J_{j}$ are the atomic number and mean ionization potential of the element $j$ and variable $k_{j}$ depends on $Z_{j}$.

The expression for $\frac{d E}{d S}$ is derived from the energy loss expression of Bethe [28]. The subsequent angle after collisions are calculated for elastic scattering which is a good approximation because the angle change during inelastic process is small $\left(\leq 0.1^{\circ}\right)$. The angle is determined using tabulated values of partial elastic cross-sections and a random number. Figure 5(a) shows the screen shot of the 2 dimensional projection of the electron trajectories generated by the program.

Once the electron trajectories are simulated, various useful distributions can be obtained, such as the distribution of maximum depth of the electrons, energy distribution of the backscattered electrons, and x-ray generation. The x-ray generation profile was used to visualize how the x-rays coming off the detector distribute themselves inside the sample. The generation of x-rays at any point is directly proportional to the number of electrons with sufficient energy to ionize the atoms. The complete details can be found in Hovington et al [29]. The software generated the x-rays and compiled the results in the classical $\phi(\rho z)$ model. The user has the flexibility to choose the incidence angle of the beam, beam energy, diameter and sample geometry, etc.

The x-ray generation output from CASINO is a dataset representing an intensity profile with an arbitrary normalization; the relevant aspect being the relative intensity with depth. Since an analytical expression for profile is required for the x-ray fitting 
procedure, various functional forms were investigated to match the dataset. In the past, various analytical expressions have been suggested with different degrees of agreements with the experiments, including shifted gaussian and exponential functions. The model that gave the best results over a wide range of electron incident angles is the Philibert expression which is a constrained sum of two exponentially decaying functions [30, 31]. For very small grazing angles, as in our case, the profile could be satisfactorily fitted to an exponential decay, similar to the Philibert model, as the peak of the distribution falls dramatically within a fraction of an angstrom from the surface. The following functional form was used to analytically model the CASINO output:

$$
I_{x-\text { ray }}(z)=y 0+A 1 \exp (-z / t 1)+A 2 \exp (-z / t 2),
$$

where $y 0, A 1, A 2, t 1, t 2$ are the fitting parameters.

Recall that Equation 26 in section 2.1 needed a correction to account for the nonuniformity in x-ray generation with depth. With this correction, the revised expression is

$$
I(\phi) \propto \int_{0}^{d_{n}} I_{x-\text { ray }}(z)\left|E_{n}^{\text {Total }}(z, \phi)\right|^{2} d z .
$$

Equation (35) is the final equation that accounts for the interaction of the electrons inside the solid and the propagation and attenuation of the x-rays generated. All the subsequent analyses carried out to explain the data are based on this equation.

(a) 


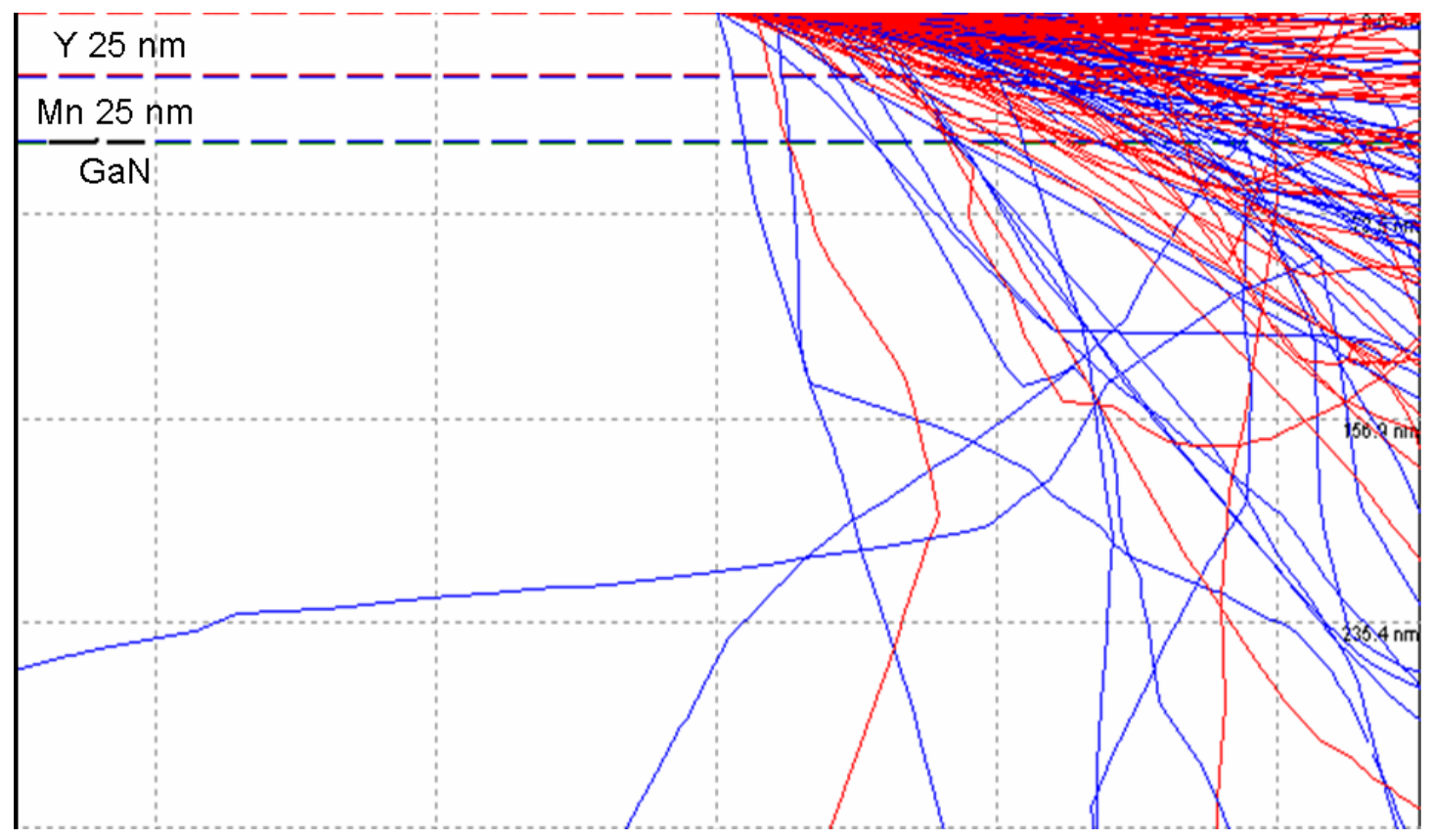

(b)
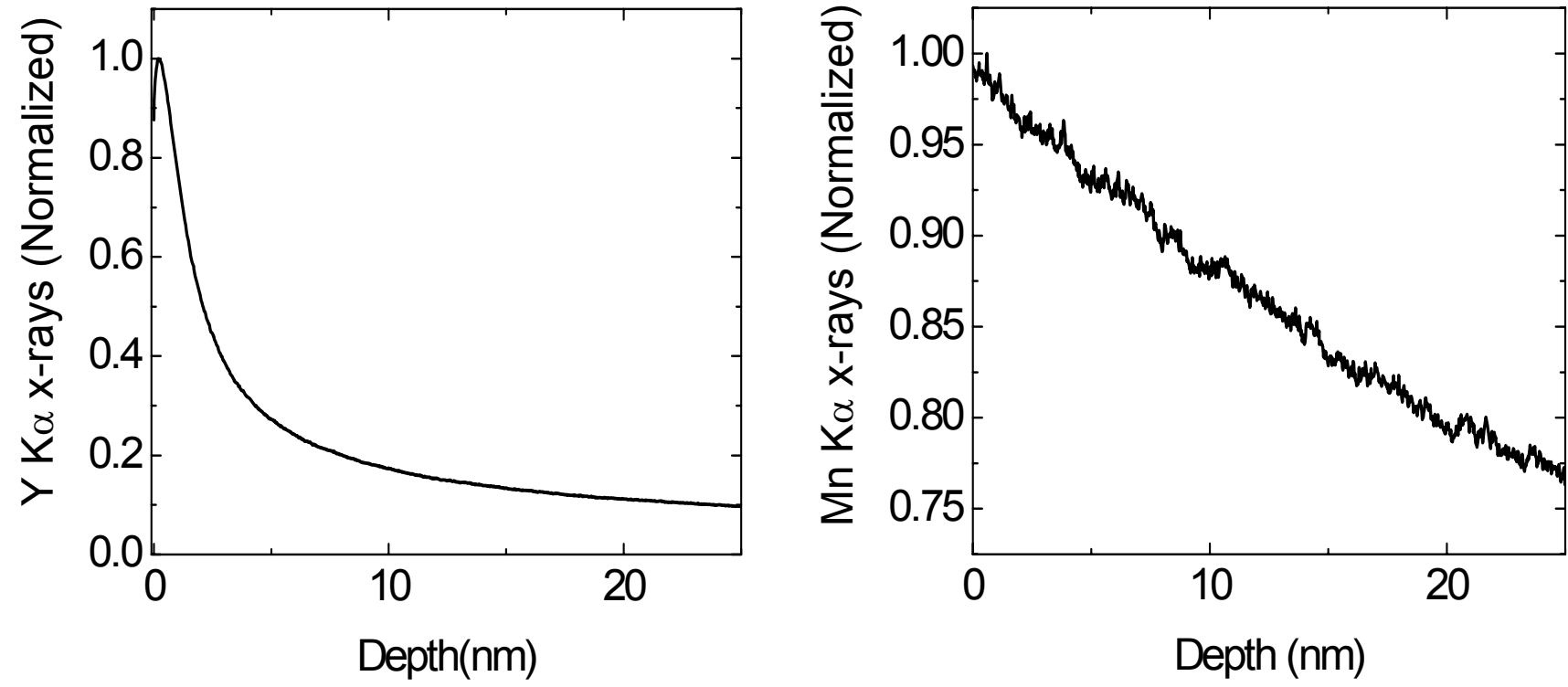

Figure 5

(a) The electron trajectories in $\mathrm{Y}$ on $\mathrm{Mn}$ on GaN sample obtained from CASINO for an electron beam angle of incidence of $2.7^{\circ}$ from sample surface and energy of $25 \mathrm{keV}$.

(b)The X-ray depth profile inside $\mathrm{Y}$ and Mn layers based on the trajectories in (a). 


\subsection{Roughness Consideration}

The formalism discussed in section 2.1 for modeling the angular dependence in RHEED-TRAXS experiments by calculating the reflectivity using Fresnel's laws and Parratt's recursive approach assumes ideal, smooth surfaces. But all surfaces have finite roughness values that affect the angular dependence and should be taken into account to match the actual conditions as closely as possible [32-34]. The roughness could be introduced in the analysis by diminishing the reflectance from the interfaces according to their respective roughness values.

Recall that Fresnel's reflectivity coefficient is $F_{n, n+1}=\left(f_{n}-f_{n+1}\right) /\left(f_{n}+f_{n+1}\right)$. The expression holds for perfectly smooth surface. Rough surfaces can be treated by the introduction of a roughness modeling factor, $Q_{n}$, in the Fresnel's reflectance coefficients.

There are various models that describe $Q_{n}$ that are not computationally intensive and give results very close to more complete treatments for small roughness $(\leq 10 \mathrm{~nm})$. The factor $Q_{n}$ was introduced as

$$
F_{n, n+1}^{\text {Rough }}=Q_{n} \cdot F_{n, n+1}^{\text {Flat }}
$$

where

$Q_{n}=1$ for an ideally smooth surface,

$Q_{n}=\exp \left(-2 \sigma_{n}^{2} k_{n}^{2}\right)$, the Debye-Waller factor,

$Q_{n}=\exp \left(-2 \sigma_{n}^{2} k_{n} k_{n+1}\right)$, the Nevot-Croce factor,

where $\sigma$ is the root-mean-squared value of the vertical roughness and $k_{n}$ and $k_{n+1}$ are the wave vectors in $n$th and $(n+1)$ th layer, respectively [35-38]. The expressions for $Q_{n}$ were derived from more rigorous theories based on the assumption of a Gaussian 
distribution of the vertical roughness. The non-gaussian behavior of the surface roughness has been investigated and modeled, but deviates from a Gaussian approximation only for very large roughness values $(>10 \mathrm{~nm})$ [39] and was not used in the analysis. After incorporating $Q_{n}$ in the reflectivity coefficient, the rest of the analysis could be carried out as before. The affect of the roughness was to reduce the recorded intensity as it reduced contribution of the reflected wave from the interface(s). The effect was more prominent around the critical angle where most of the intensity came from close to the surface. The effects would be illustrated later on with the simulation curves.

\subsection{Simulation results}

The following section describes the simulation of various single layer and multilayered structures of $\mathrm{Y}, \mathrm{Mn}, \mathrm{GaN}$ and $\mathrm{YMnO}_{3}$ and discusses the effects of material, thickness and roughness on the angular dependence of grazing exit fluorescence.

Mentioned previously, the complex index of refraction in the x-ray regime can be

written as $1-\delta-i \beta$ which leads to the angle of refraction $\phi_{2}=\left(\phi_{1}^{2}-2 \delta_{2}-2 i \beta_{2}\right)^{1 / 2}$ (Figure 2). The values of $\delta$ and $\beta$ can be calculated from

$$
\begin{aligned}
& \delta=4.1516 \times 10^{-4} \frac{\rho}{M \cdot E^{2}} \sum_{j=1}^{N} C_{j} f_{1 j} \\
& \beta=4.1516 \times 10^{-4} \frac{\rho}{M \cdot E^{2}} \sum_{j=1}^{N} C_{j} f_{2 j}
\end{aligned}
$$

where $\rho$ is the density in $\mathrm{g} / \mathrm{cm}^{3}, E$ is the energy of $\mathrm{x}$-ray radiation in $\mathrm{keV}$ and $M$ is the molar weight $(\mathrm{g} / \mathrm{mol})$ of a compound of $N$ different atoms. $C_{j}$ are the number of atoms of 
type $j$ per molecule and $f_{j}=f_{1 j}+f_{2 j}$ are the atomic scattering factors for atoms of type $j$.

For $\phi_{1}=\phi_{\text {critical }}, \phi_{2}=0$. So the critical angle can be approximated as $\phi_{\text {critical }} \approx \sqrt{2 \delta_{2}}$ as $\beta$ is approximately two orders of magnitude smaller than $\delta$. Since $\delta_{\mathrm{Mn}}>\delta_{\mathrm{Ga}}>\delta_{\mathrm{Y}}$, for the layers of same thickness, the critical angle for Mn, gallium and $\mathrm{Y}$ follow the trend $\phi_{\mathrm{Mn}}>$ $\phi_{\mathrm{Ga}}>\phi_{\mathrm{Y}}$ as shown in Figure 6. The same qualitative behavior was observed in our initial experiments. The x-ray depth profile inside a single layer of Mn and $\mathrm{Y}$ are shown in Figure 7.

Before comparing the simulations with the actual experimental data, the effect of the collimating slit must also be considered. The diverging effect of the x-ray beam due to the slits was successfully simulated by a Gaussian centered at the slit and of width 0.7 mrad, which is equal to half the angular divergence of the slit. Then the total intensity at any point is the convolution of the angular dependent intensity distribution and the Gaussian function $G$. Hence, the total intensity at any angle $\phi$ is

$$
I^{\text {Total }}(\phi) \propto \frac{\int_{-\infty}^{\infty} I(\phi) G(\phi-\theta) d \theta}{\int_{-\infty}^{\infty} G(\phi-\theta) d \theta}
$$

All the simulation results that follow are derived from Eq. (42).

Figure 8(a) shows the angular dependence of Mn and its variation with Mn layer thickness. It is clear that the peak at the critical angle becomes broader as the thickness is increased until it disappears, leading to bulk-like behavior. Also, the peak position moves towards a higher angle as the thickness is increased. The same trend can be observed in the $\mathrm{Y}$ angular dependence as shown in Figure 9(a). 


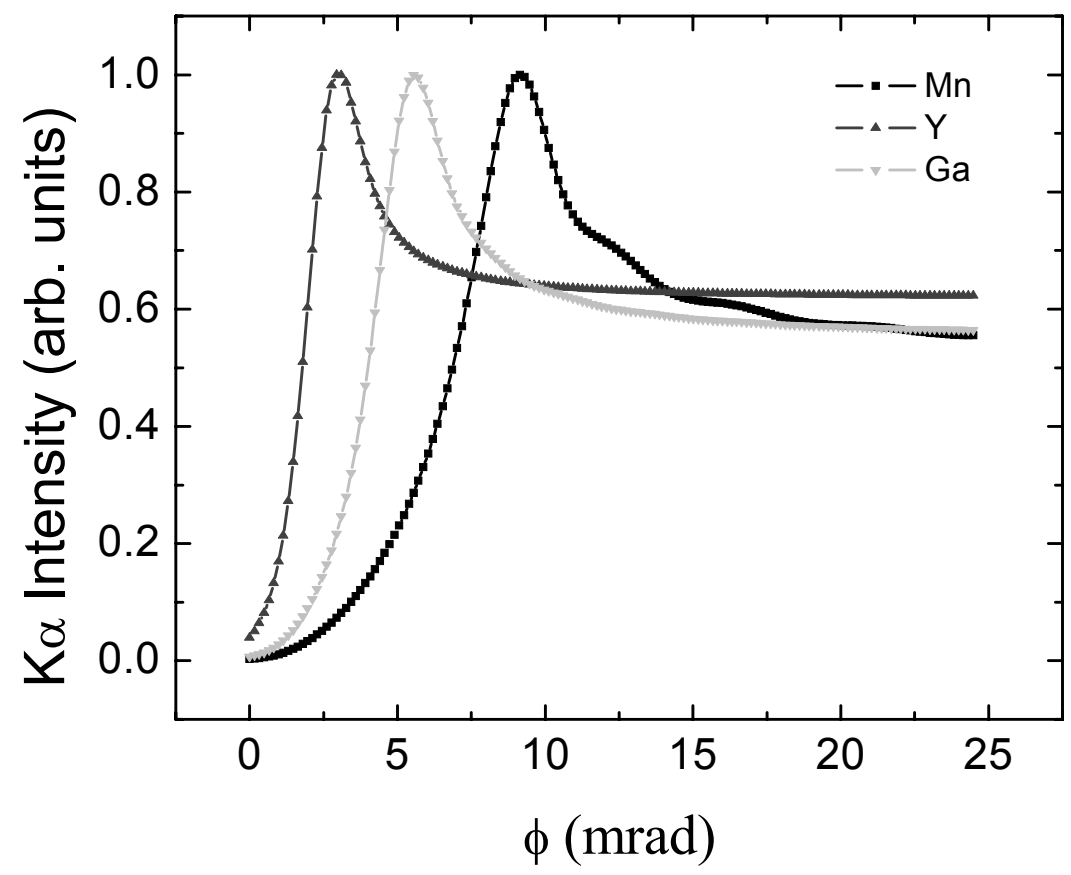

Figure 6

The simulation of angular dependence of $20 \mathrm{~nm}$ of Y, Ga and Mn on a sapphire substrate.

The position of the critical angle can be estimated from $\phi_{\text {critical }} \approx \sqrt{2 \delta_{2}}$. 

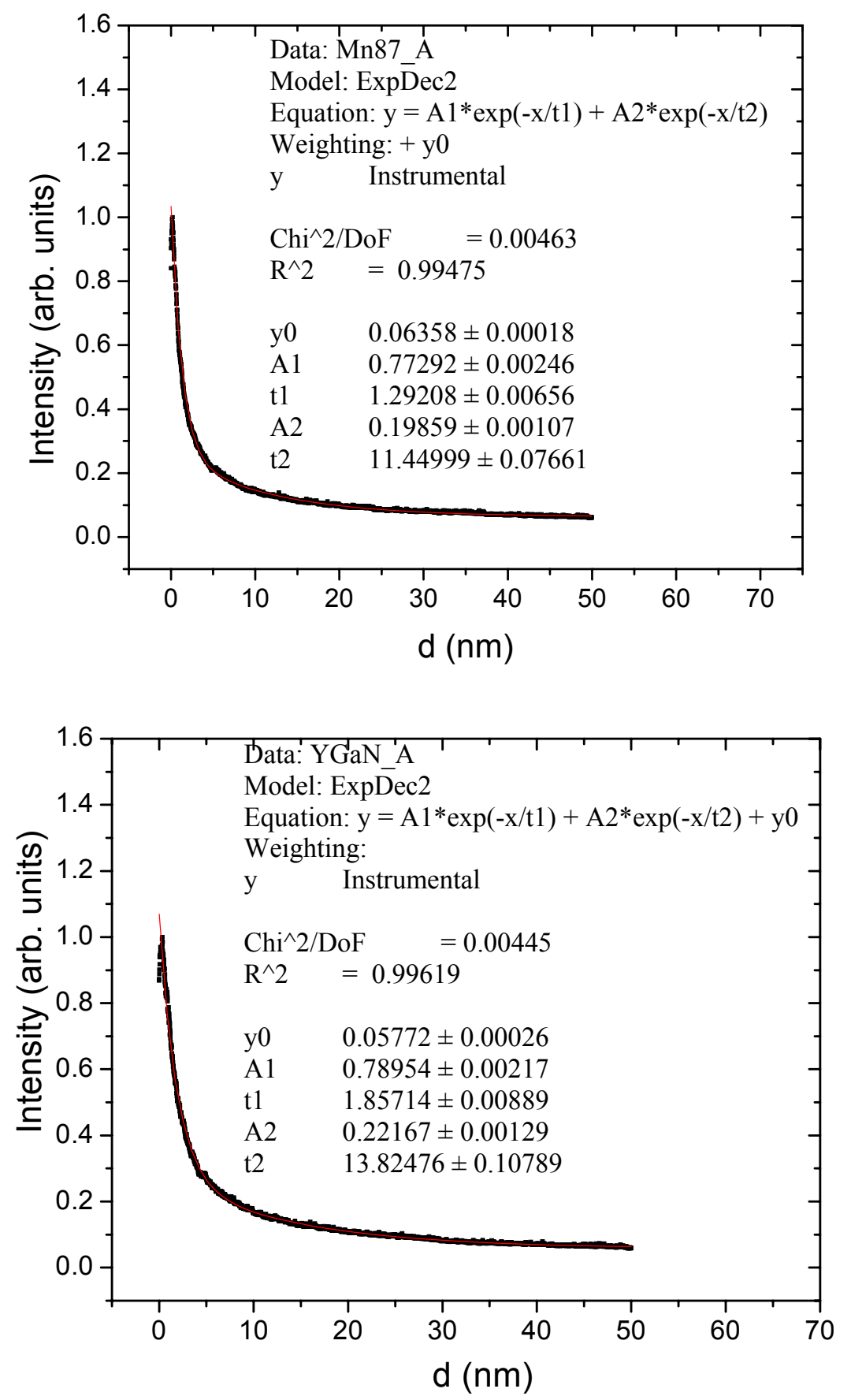

\section{Figure 7}

The x-ray depth profiles for $50 \mathrm{~nm}$ thick layers of $\mathrm{Mn}$ (a) and $\mathrm{Y}$ (b) on GaN substrate. The best fit to the simulation data was obtained with the sum of two exponential functions. 
In fact, the angular dependence of $\mathrm{GaN}$ templates always yielded the bulk-like dependence in our experiments as they had thickness of the order of a few microns. At such thicknesses, the angular dependence is no longer sensitive to the thickness.

Figure 8(b) \& Figure 9(b) shows the effect of surface roughness on the angular dependence of Mn and Y. Intuitively, one would expect the roughness to diminish the intensity from a flat surface. It can be seen in the figure that the most observable effect of increasing roughness is to decrease the peak intensity without changing its shape and position, and has a diminishing effect on the high angle part that gets most of the contribution from deeper in the layer. 
(a)

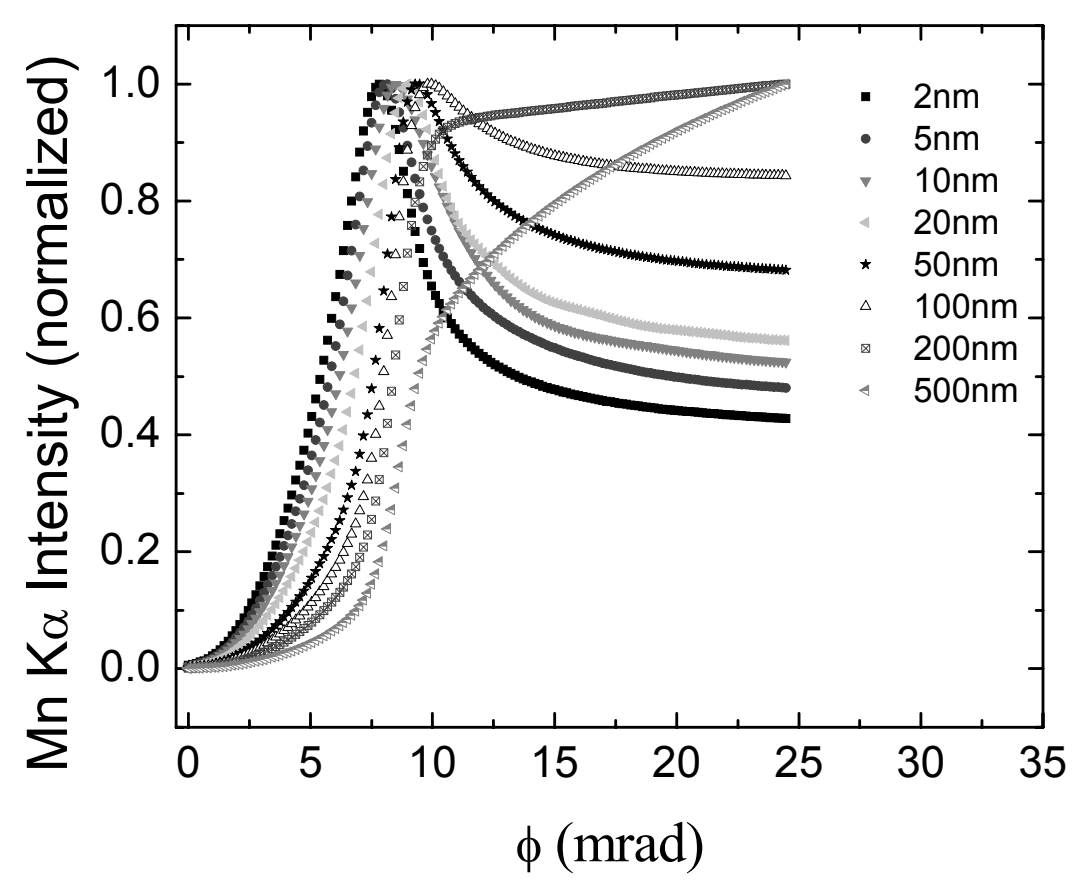

(b)

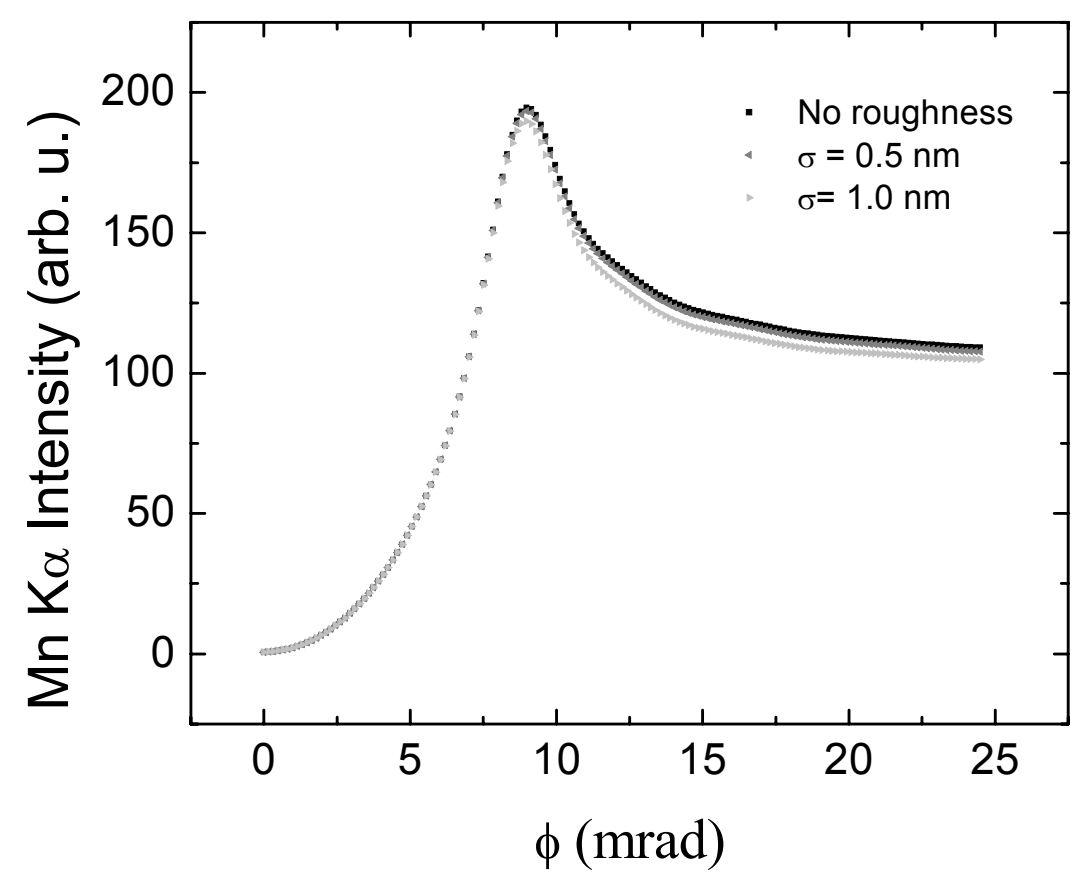

Figure 8

Simulation results for Mn for various thickness values (a) and roughness values for 20 nm thick Mn layer (b). 
(a)

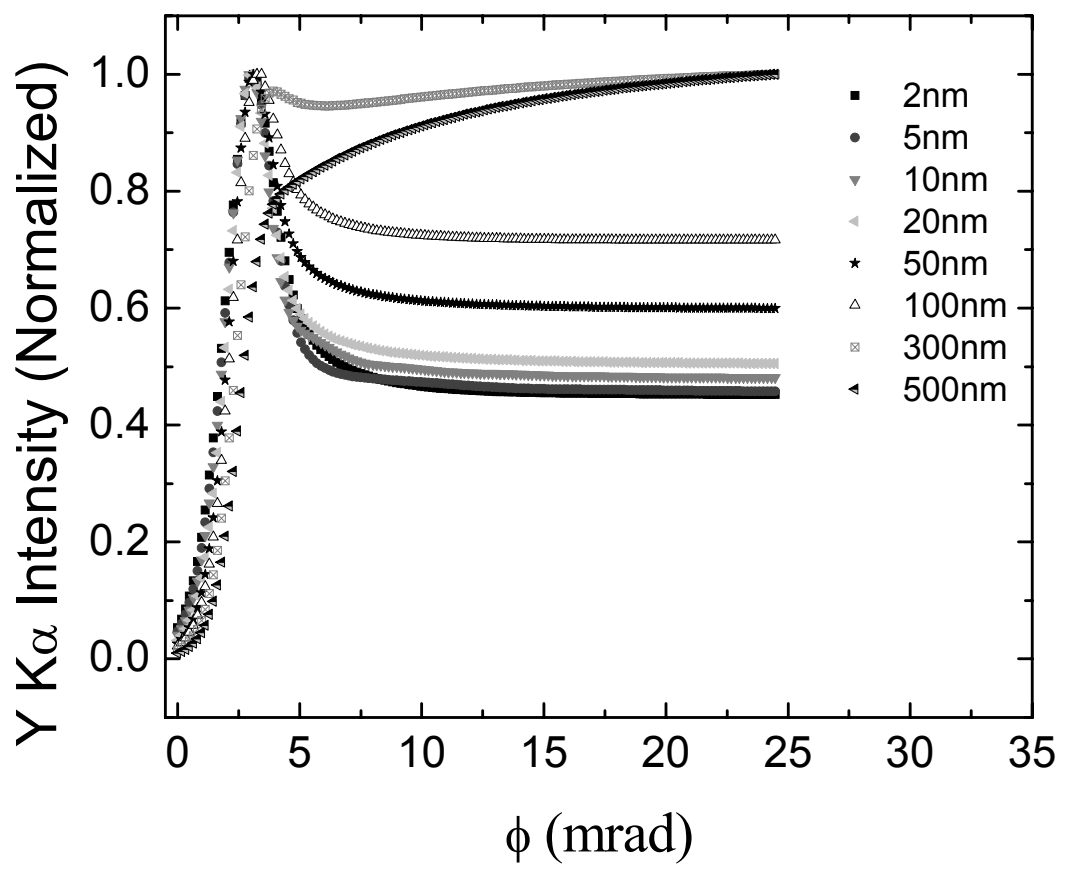

(b)

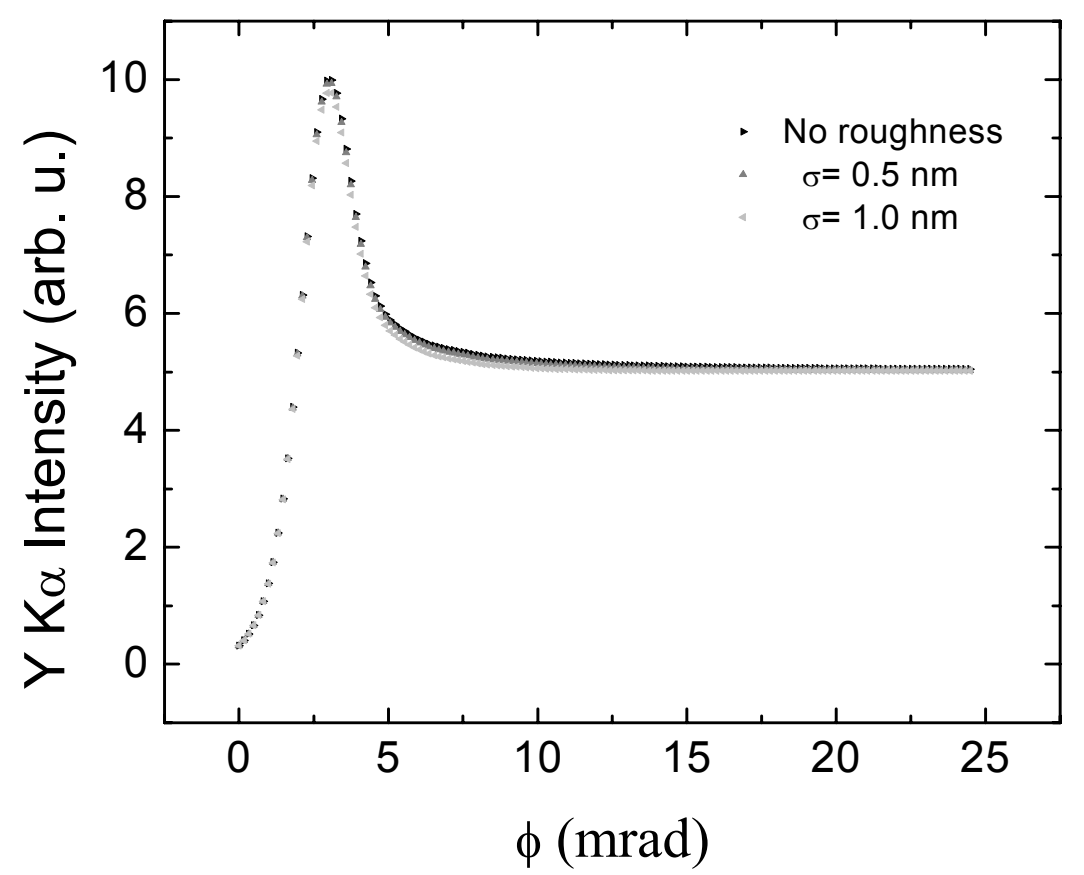

Figure 9

Simulation results for $\mathrm{Y}$ for various thickness values (a) and roughness values for $20 \mathrm{~nm}$ thick Y layer (b). 


\section{Experiment}

The experiments were performed in a Molecular Beam Epitaxy (MBE) chamber dedicated to growing ferroelectric oxide, $\mathrm{YMnO}_{3}$ (Figure 10 (a)). Single layers of $\mathrm{Mn}$ and $\mathrm{Y}$ as well as bilayers containing $\mathrm{Y}$ on $\mathrm{Mn}$ and $\mathrm{Mn}$ on $\mathrm{Y}$ were deposited on the $\mathrm{GaN}$ and capped with Al to prevent oxidation once the sample was removed from the system for ex-situ analysis. Y and Mn were deposited on substrates at room temperature with K-cell temperatures of $1427{ }^{\circ} \mathrm{C}$ and $820^{\circ} \mathrm{C}$, respectively. The approximate growth rate was 0.09 $\AA \sec ^{-1}$ for $\mathrm{Mn}$ and $0.17 \AA \mathrm{sec}^{-1}$ for $\mathrm{Y}$ as measured using a quartz crystal monitor. Al was deposited on top of Y/Mn layer(s) using electron beam deposition with a growth rate of approximately $0.92 \AA / \mathrm{sec}$. The films were grown so that they were thick enough to give a statistically significant fluorescence signal and easily analyzable using x-ray reflectivity measurements.

\section{MBE Chamber}

The oxide chamber is connected to a cluster tool that is also connected to a nitride MBE chamber, a metals MBE chamber and an analysis chamber. A load lock for transferring samples and a sample storage unit are mounted on the cluster tool for transferring. The set up, except for the metals chamber, was custom-built as a part of a multifunctional project aimed at studying in details the epitaxial growth and physical properties of oxide multiferroic materials on GaN. This is a unique facility that allowed the transfer of samples from one chamber to another for subsequent growth/analysis without breaking the vacuum and hence, without compromising the interface quality.

The oxide chamber is equipped with $30 \mathrm{keV}$ RHEED gun by Staib Instruments Inc. with enough energy to excite the $\mathrm{Y} \mathrm{K} \alpha$ peak at $14.96 \mathrm{keV}$. It had an emission current 
capability of up to 100 microamperes, but currents in excess of 10 microamperes was rarely used. It also has a unique custom-built current feedback control which was designed to produce very stable emission currents. With this feedback control, current stability of hundredth of a microampere was achievable for 1-5 $\mu \mathrm{A}$ emission currents.

\section{RHEED-TRAXS setup}

The x-ray detector for RHEED-TRAXS sat on an arm about 15" outside the chamber on a $23 / 4$ " port. The port is at a right angle to both the RHEED gun and the fluorescent screen. The arm has bellows for vertical movement of approximately 1 " that could extend an angle of about $2.13^{\circ}$ with respect to the sample surface. The RHEEDTRAXS arm was separated from the chamber by a gate valve and was differentially pumped which allowed for easy assembly of the detector without venting the system. The detector was screwed on the end of the arm with a rubber gasket seal. The detector assembly and the differential pumping line was separated from the rest of the system by a $1 / 4$ " hole in a solid copper gasket so that system pressure would not go up considerably when the port is open to the system(Figure $10(b)$ ).

The port was centered with the focal point of the source k-cells so the center of it looked straight at the sample. RHEED gun port was at $1^{\circ}$ angle with respect to the sample plane. The actual angle of the electron beam with respect to the sample was determined by measuring how far the beam hit the RHEED screen from its center once the sample was raised, as the distance of the RHEED screen from the sample position is known. Once the angle was determined, the sample was lowered until a RHEED pattern was obtained on the screen to ascertain that the beam is right on the sample. The window at the bottom of chamber was also used to look at the cathodoluminescence from the sample 
to find the beam position. The angle used for all the experiments was $2.8^{\circ}$. The diameter of the beam was about $1 \mathrm{~mm}$ and it had a footprint of the order of a centimeter on the sample.

The base pressure in the MBE was $\sim 10^{-9}$ Torr and the base pressure at the differentially pumped detector was $\sim 10^{-7}$ Torr. The detector assembly was hermetically sealed to hold high vacuum. The schematics for RHEED-TRAXS setup are shown in Figure 11.

\section{Sample preparation}

All the samples were grown on hydride vapor phase epitaxy (HVPE) GaN templates on sapphire substrate. The GaN templates were about 4 microns thick. All the substrates were degreased using Trichloroethylene (TCE), Acetone and Methanol heated at $\sim 60^{\circ} \mathrm{C}$ and blown dry with UHP nitrogen. Some of the substrates were etched in $50 \%$ HCL for 5 minutes, rinsed with DI water and dried using UHP nitrogen. The samples were mounted on an alumina block using an Indium-Tin eutectic. 

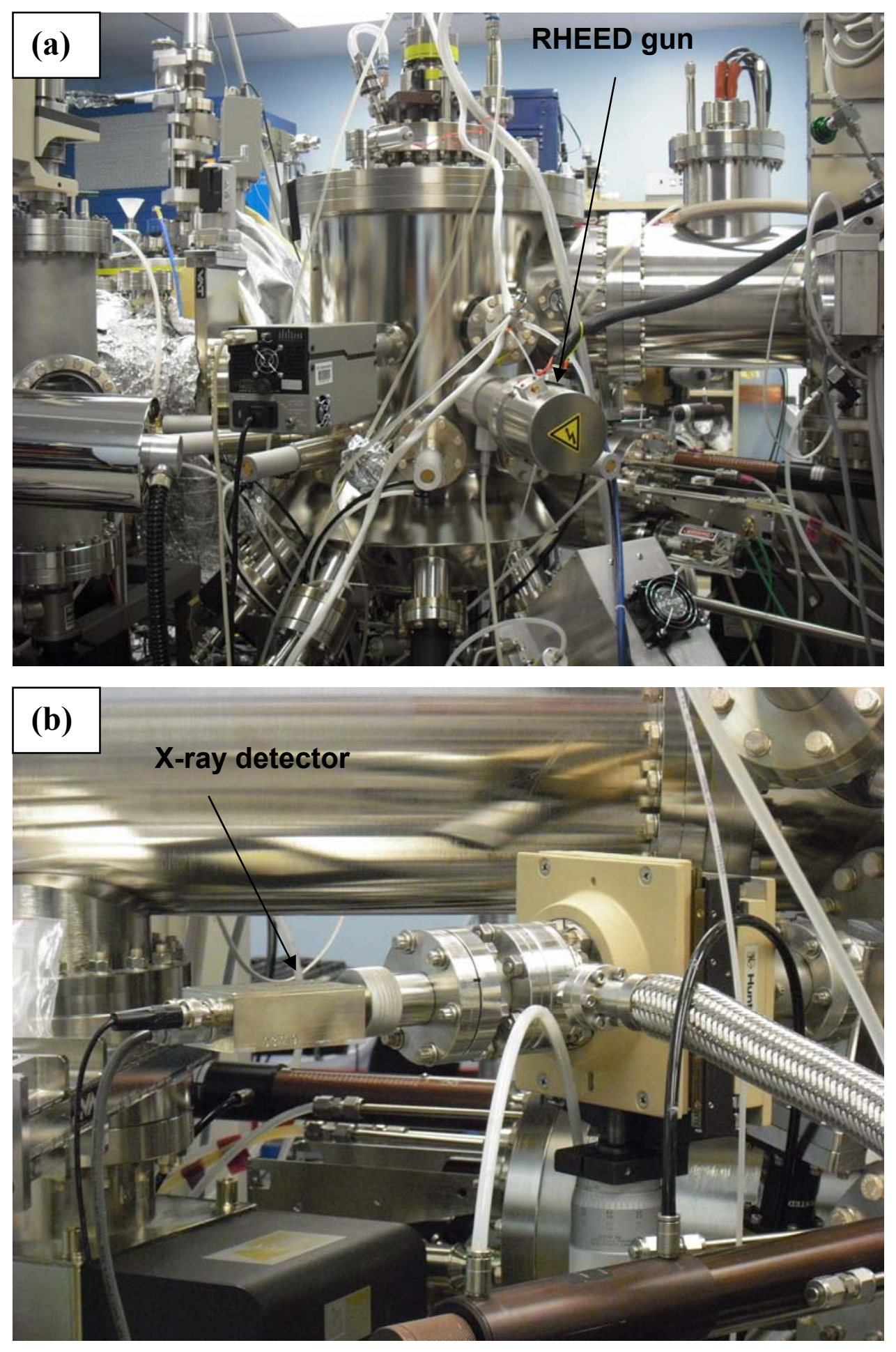

Figure 10

(a) The oxide MBE system for growing YMnO3. (b) RHEED-TRAXS arm with Si X-ray detector at the end of bellows arm. 


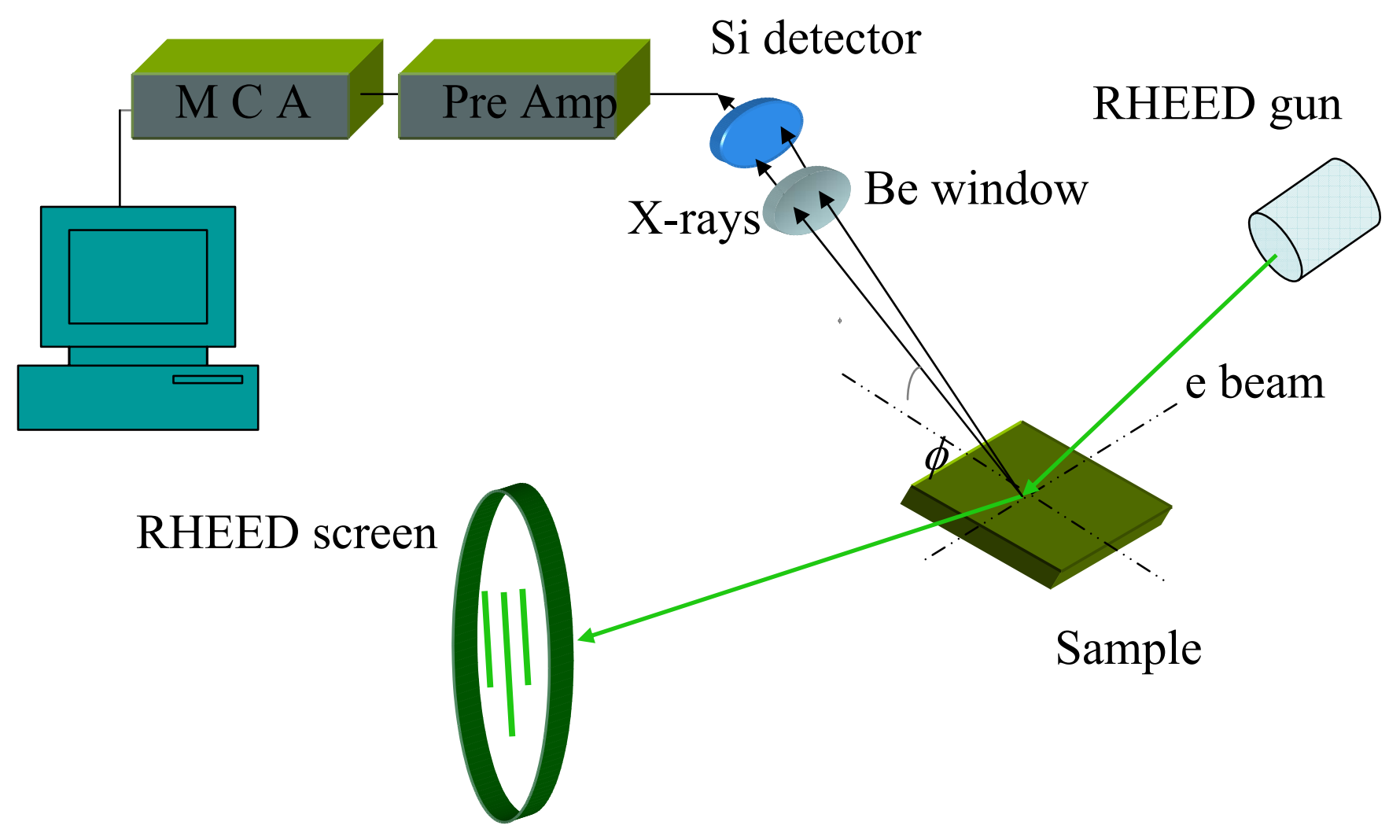

Figure 11

The schematic of RHEED-TRAXS setup. Si detector and pre-amplifier are contained in a single unit that sits at the end of the RHEED-TRAXS arm. The data are collected on a computer by a multichannel analyzer (MCA). 


\subsection{X-ray Detection}

X-ray fluorescence from the sample was detected using a silicon PIN diode. X-rays hitting the detector can deposit their energy by Compton scattering or by the photoelectric process. For the energies of interest, the photoelectric process was the dominant affect. Inside the detector, the photoelectric process creates electron-hole pairs with electrons in the conduction band. The detector is biased to cause the charge pairs to drift towards the electrodes and generate a current pulse; the amplitude size of the pulse is proportional to the energy of the x-ray photon. This is the basic principle behind energy-dispersive $\mathrm{x}$-ray detection. The detector was thermoelectrically cooled to reduce the thermally excited electron hole pair and thus the noise.

\section{Instrumentation}

The set up for x-ray detection was purchased from Amptek Incorporated and included an X-ray detector and preamplifier module (XR100-CR), a digital pulse processor and multichannel analyzer module (PX4) (Figure 12), and interfacing software [40]. The XR100-CR detector had a $5 \mathrm{~mm} \mathrm{X} 5 \mathrm{~mm}$ wide, 500 microns thick silicon PIN diode as the active area for $\mathrm{x}$-ray detection. There was a 0.5 mil thick Beryllium window in front of the diode that hermetically sealed on the mount shown in Figure 12(c). Be has low ionization energy $(0.1 \mathrm{kV})$ and did not interfere with the $\mathrm{x}$-rays of interest.

The detector was thermoelectrically cooled with a feed back loop to maintain constant temperature of about $200 \mathrm{~K}$. A temperature sensor sat very close to the detector for accurate measurement. 
(a)

(b)
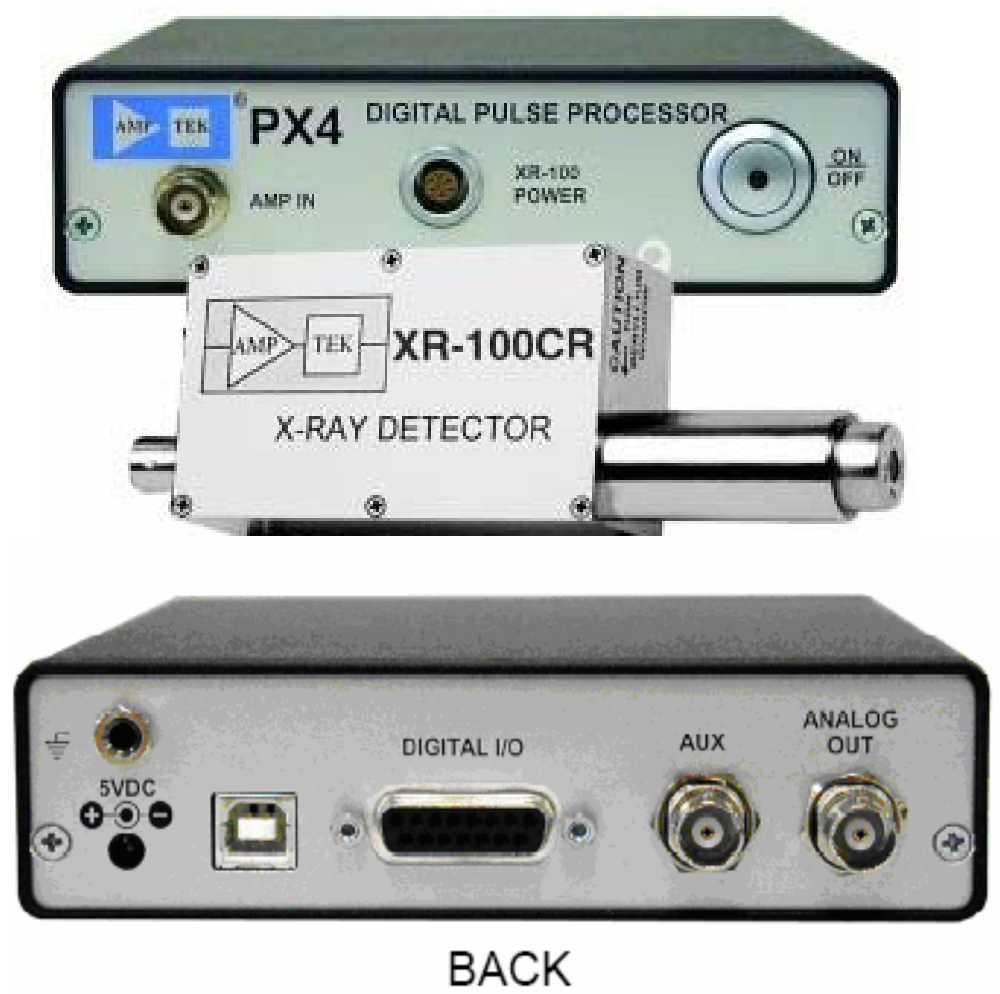

(c)

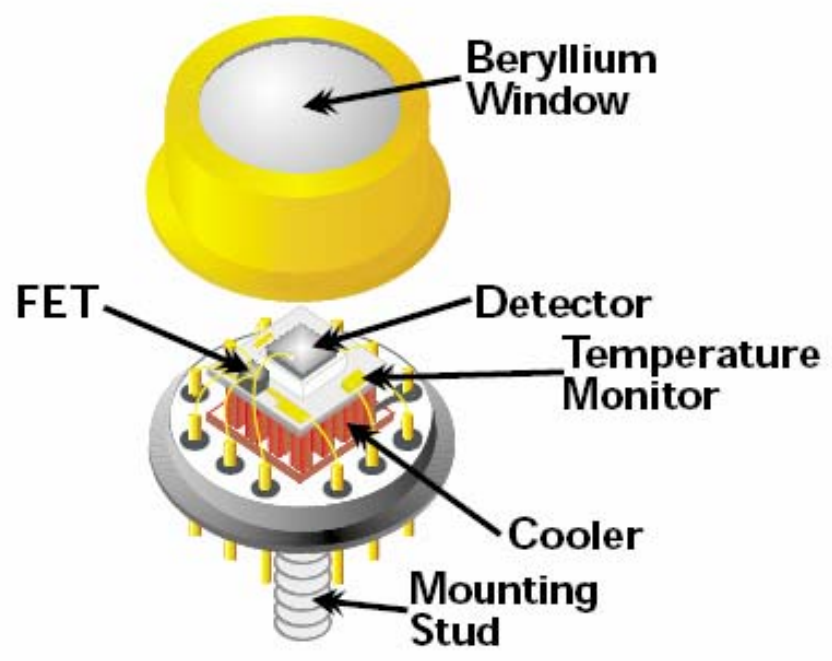

Figure 12

(a) XR100 x-ray detector and PX4 multichannel analyzer, (b) PX4 from the back and (c) the detector assembly [40]. 
The photoelectric efficiency curve for the detector is shown in Figure 13. Figure 13 combines the affect of both Be window and the interaction in the detector. The low energy portion of the curve is dominated by the absorption in the beryllium window and high energy part is dominated by the active depth of the detector. The other characteristics, such as energy resolution and peak to background ratio, are dependent upon the peaking time and the count rate. This can be best explained after discussing the pulse processing module.

The charge pulse generated in the active diode area was converted into a voltage signal by the field effect transistor (FET) preamplifier before feeding into PX4 circuitry for further processing. The preamplifier had a gain of $-1 \mathrm{mV} / \mathrm{keV}$. Hence, a photon of 10 $\mathrm{keV}$ produced a voltage step of $-10 \mathrm{mV}$. The voltage steps were processed by PX4 electronics to determine the pulse height for accurate binning by a multichannel analyzer (MCA).

The pulse processor in the PX4 has six main function blocks: an analog prefilter, an analog to digital converter (ADC), a digital pulse shaper, pulse selection logic, and interfacing hardware and software (Fig. 9). The analog prefilter prepares the signal for accurate digitization by applying appropriate gain, offset and inversion, if necessary, to utilize the dynamic range of ADC. ADC digitizes the output of the analog prefilter at 20 MHz. The digital signal is then sent to digital pulse shaper. Figure 14 shows the output signal pulse during various stages of processing.

The output from ADC flows through two parallel channels, a slow channel and a fast channel in the digital pulse shaper module. The slow channel has a long peaking time 

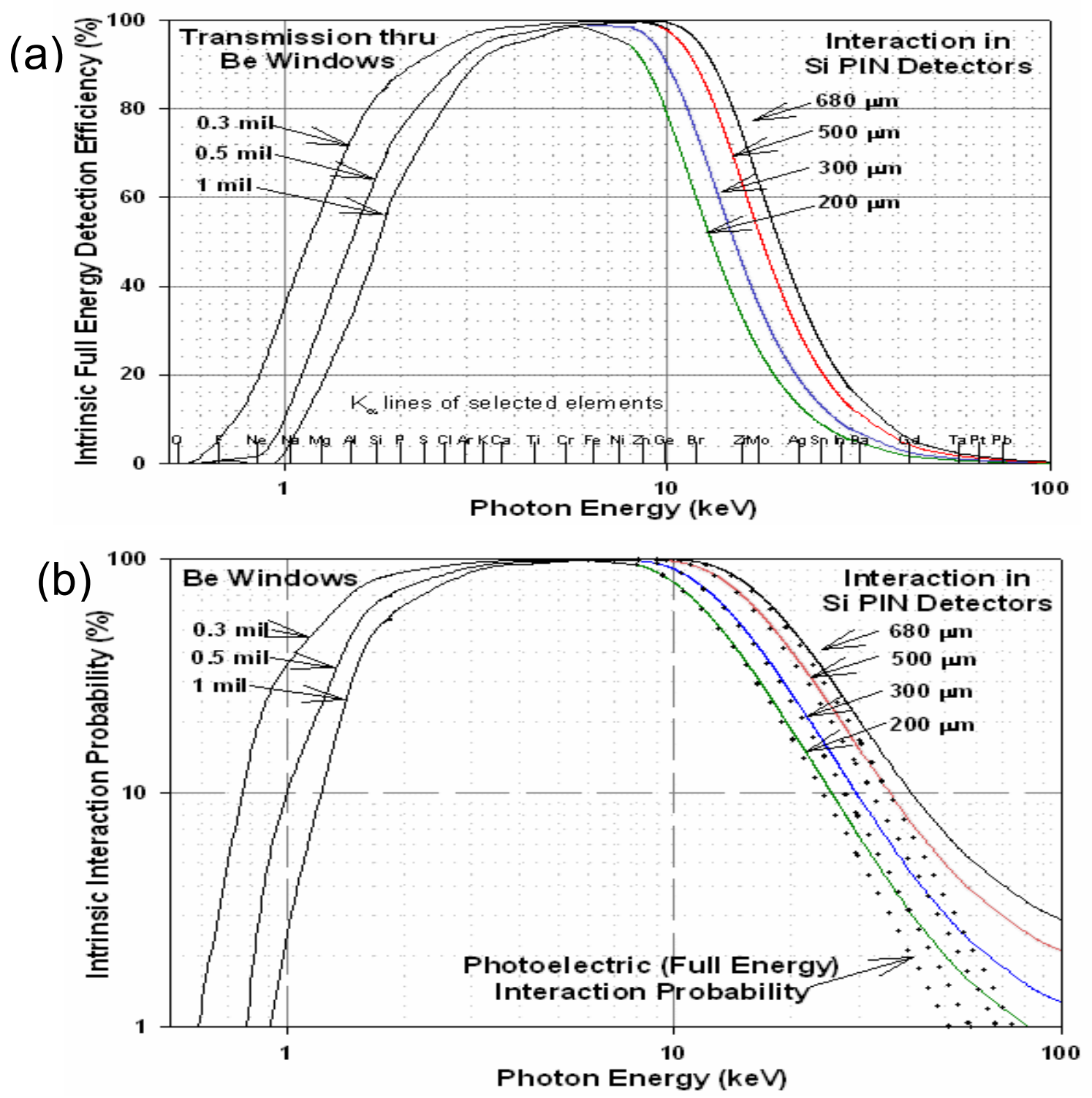

Figure 13

(a)The intrinsic full energy detection efficiency for Si-PIN detectors. This efficiency corresponds to the probability that an X-ray will enter the front of the detector and deposit all of its energy inside the detector via the photoelectric effect.

(b) The probability of a photon undergoing any interaction in Si-PIN detectors, along with the probability of a photoelectric interaction which results in total energy deposition. The photoelectric effect is dominant at low energies but at higher energies above about $40 \mathrm{keV}$ the photons undergo Compton scattering [40]. 
and is optimized to minimize noise and get accurate peak height. The counts for the spectrum come from this channel (Figure 15).

Peaking time is the time required for the shaped pulse to go from the baseline to the peak. Shaping time, one of the adjustable parameters in the software, is 2.2 times the peaking time and is related to the time constant of the shaping amplifier. Also, dead time is the duration of time following a valid event during which a subsequent event will not be recorded.

The fast channel has much shorter peaking time $(0.4 \mu \mathrm{sec})$ than the slow channel and is used to detect the actual incoming counts and detect pile up pulses in the slow channel. If two pulses are too close, their pile up may lead to a shaped pulse that is the sum of their amplitudes and a false count will be recorded by MCA. Figure 15 shows how slow and fast channels work together to generate the spectrum.

The fast channel has a dead time, $\tau_{\text {Fast }}$, equal to the peaking time. Because of the finite dead time, the count rate recorded by the fast channel, $R_{\text {Fast }}$, is different from the actual incoming rate, $R_{I n}$. This can be expressed by

$$
\begin{aligned}
& R_{\text {Fast }}=R_{\text {In }} \exp \left(-R_{\text {In }} \tau_{\text {Fast }}\right), \\
& R_{\text {In }} \cong \frac{R_{\text {Fast }}}{1-R_{\text {Fast }} \tau_{\text {Fast }}} .
\end{aligned}
$$

Figure 16 shows the graph of fast channel measured count rate as a function of the true incoming count rate. In our experiments, the measured count rate was approximately $10^{3}$ counts per second, so the measured rate is equal to the true incoming rate, to a very good approximation, i.e.,

$$
R_{\text {In }} \cong R_{\text {Fast }} \text {. }
$$


(a)

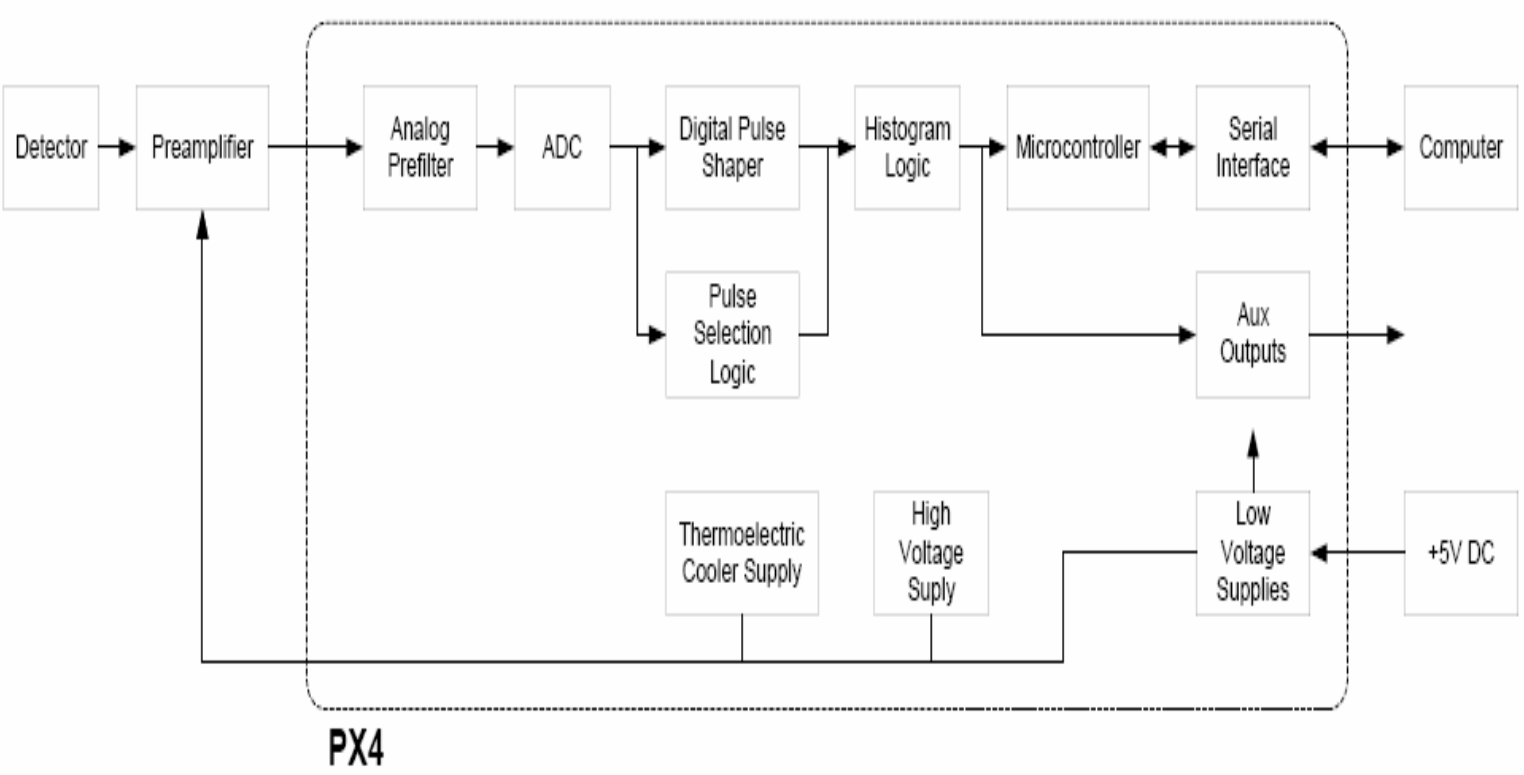

(b)

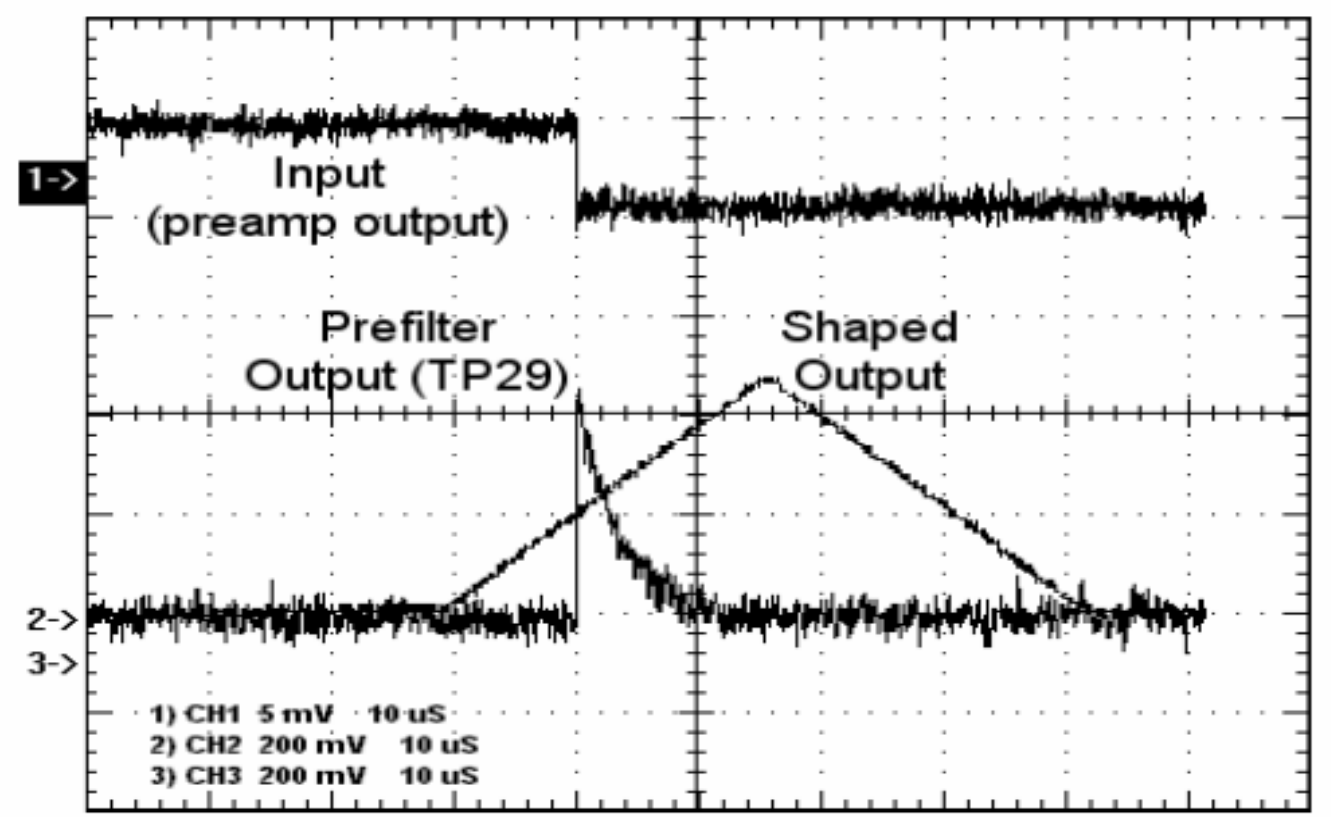

Figure 14

(a) The block diagram of x-ray pulse processing using XR100 CR detector and PX4

pulse processor. (b) The signal output from various modules of the pulse processing circuitry. Preamplifier output is the output from preamplifier and input to analog prefilter; prefilter output is the output from analog prefilter; shaped output is the output from digital pulse processor 40 . 


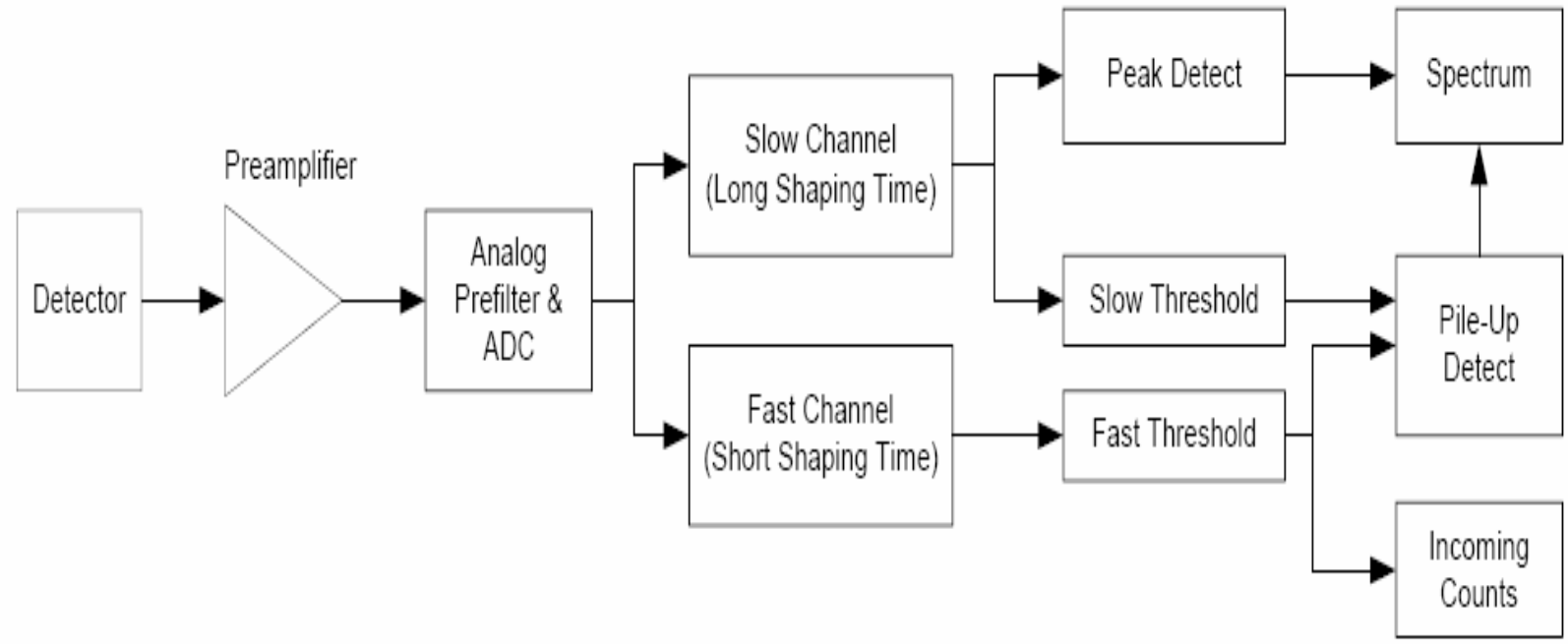

Figure 15

Signal processing through slow and fast channels to produce the spectrum [40]. 
For the slow channel, the count rate is dependent on whether pile up rejection (PUR) is on or off. With PUR on, the dead time is closer to $\tau_{\text {peak }}$, but it's greater than $\tau_{\text {peak }}$ with PUR on.

$$
\begin{gathered}
R_{\text {PUR_off }}=R_{I n} \exp \left(-R_{I n} \tau_{\text {peak }}\right) \\
R_{\text {PUR_on }}=R_{I n} \exp \left(-2 R_{I n}(19 / 16) \tau_{\text {peak }}\right)
\end{gathered}
$$

Figure 17 shows input count rate (ICR) versus output count rate (OCR) recorded by the slow channel with PUR on and with different shaping times. For count rates of the order of $10^{3}$, we can approximate the recorded count rate as the actual incoming count rate and have direct correspondence between the recorded value and the concentration of the analyte.

The selection of appropriate peaking time is a trade-off between faster counting and energy resolution. Lower peaking times give a linear relation between ICR and OCR for a wider range of ICR, but have worse peak resolution in the energy spectrum. The shaping time of $25.6 \mu \mathrm{s}$ was used for all data acquisitions. 


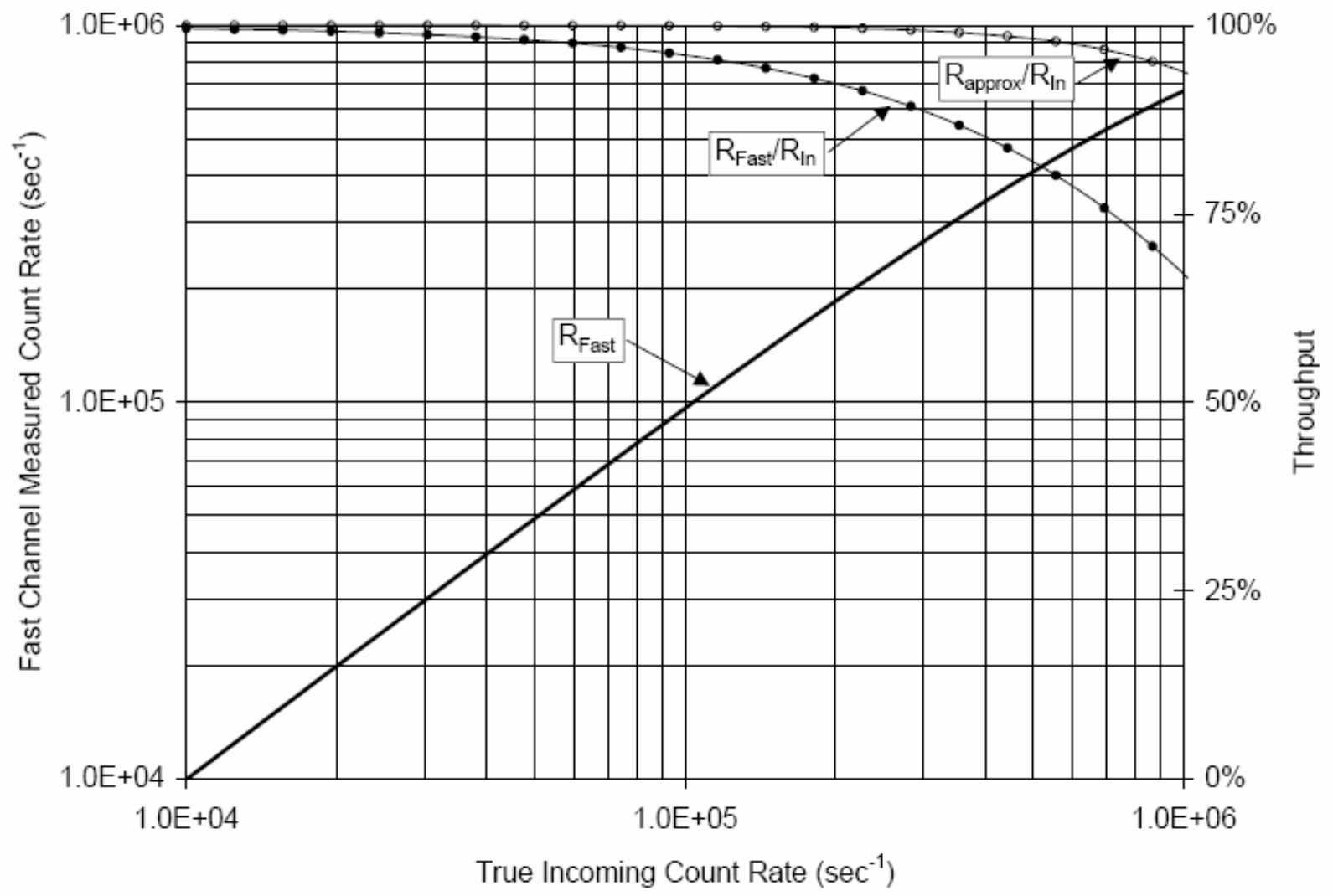

Figure 16

Throughput of the fast channel at various incoming count rates [40]. 


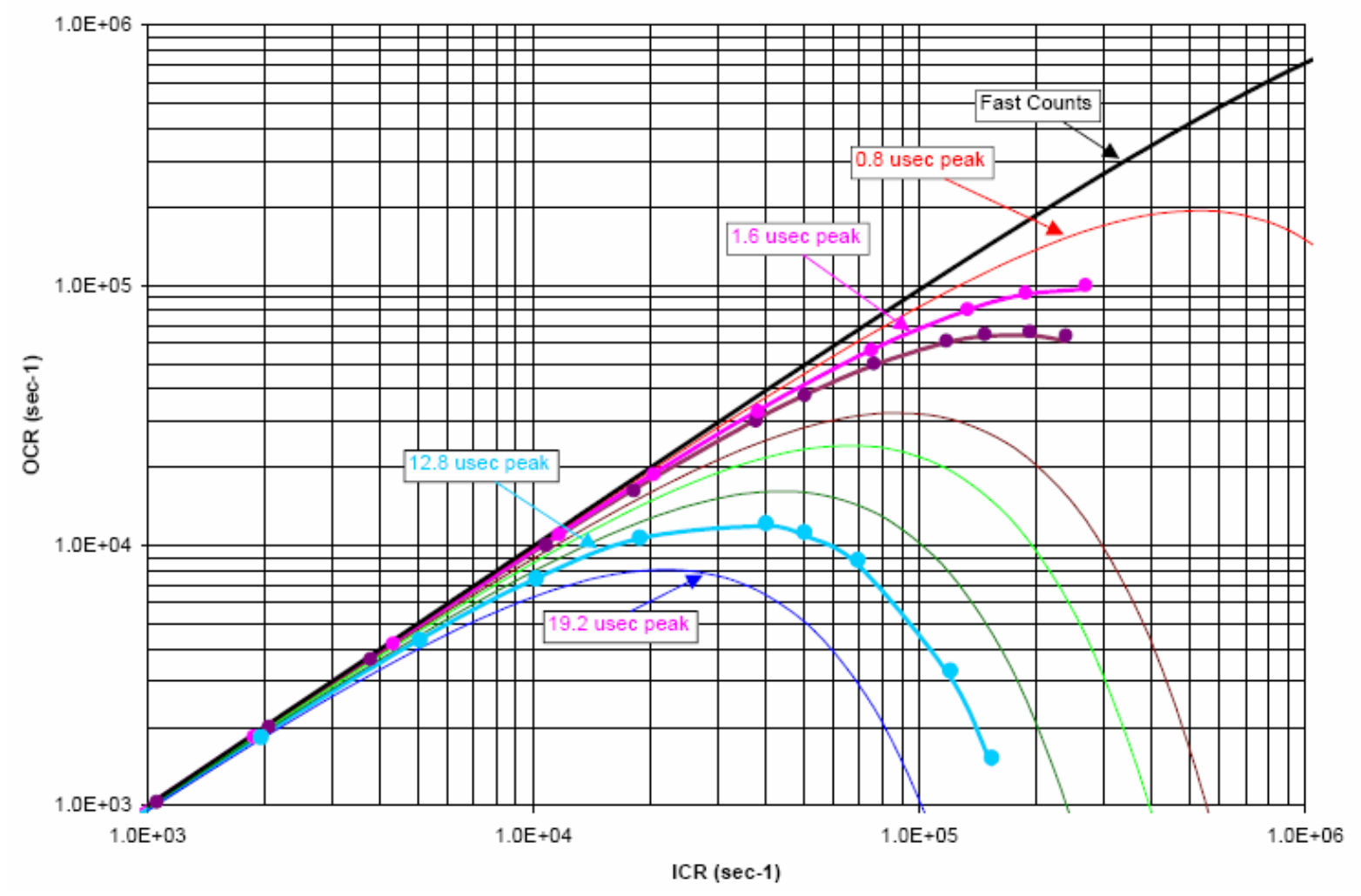

Figure 17

Input and output count rates with pile up rejection on and various peaking times [40]. 


\subsubsection{RHEED-TRAXS}

The energy dispersive spectrum obtained from PX4 is shown in Figure 18. 1024 channels were used to bin the counts from about $2 \mathrm{keV}$ to $20 \mathrm{keV}$. It shows the discrete spectrum corresponding to the characteristic energy peaks of the elements superimposed on the continuous spectrum due to bremsstrahlung radiation. Bremsstrahlung, as described earlier, can take any value from zero up to the entire energy of the electron, forming a continuous spectrum. Since it does not represent the characteristic energy of the inner shell ionization of the element(s), it should be removed from the spectrum for analytical purposes. Hence, the area of a characteristic peak above the bremsstrahlung was used for all the analyses and bremsstrahlung was treated as background.

The spectrum was sectioned into various regions containing the relevant peaks, the local background was approximated as linear and the Gaussian profile was used to fit the peak. The error associated with every count in the peak was the statistical error calculated as the square root of the number of counts. Figure 19 shows gallium and $M n K_{\alpha}$ and $K_{\beta}$ peaks, $\mathrm{Y} \mathrm{K}_{\alpha}$ peak and the best fits to them for $\mathrm{Y}$ and $\mathrm{Mn}$ thin films grown on a $\mathrm{GaN}$ substrate. For $\mathrm{Y}$, a $\mathrm{K}_{\beta}$ peak was well separated from $\mathrm{K}_{\alpha}$ peak and was sectioned off. Figure 19(c) shows the best fit to the $\mathrm{Y} \mathrm{K}_{\alpha}$ peak [41] . Only $\mathrm{K}_{\alpha}$ peaks were considered for RHEED-TRAXS analysis because of signal's higher intensity. Figure 19(d) shows peak intensity at various angles for the substrate used for Mn thin film deposition.

Once the peak areas at various angles were obtained, the data were fitted to the theoretical model using Levenberg-Marquardt algorithm and the errors for $i$ th parameter 
were calculated as $\sqrt{\frac{\sigma_{i i}^{2} \chi^{2}}{D O F}}$, where $\chi^{2}$ is the goodness of fit, $\sigma_{i i}^{2}$ is the ith diagonal element in the covariance matrix and DOF is the degrees of freedom [41] .

Once the samples were removed from the growth chamber, x-ray reflectivity measurements were performed on them to compare the results with RHEED-TRAXS experiments. 


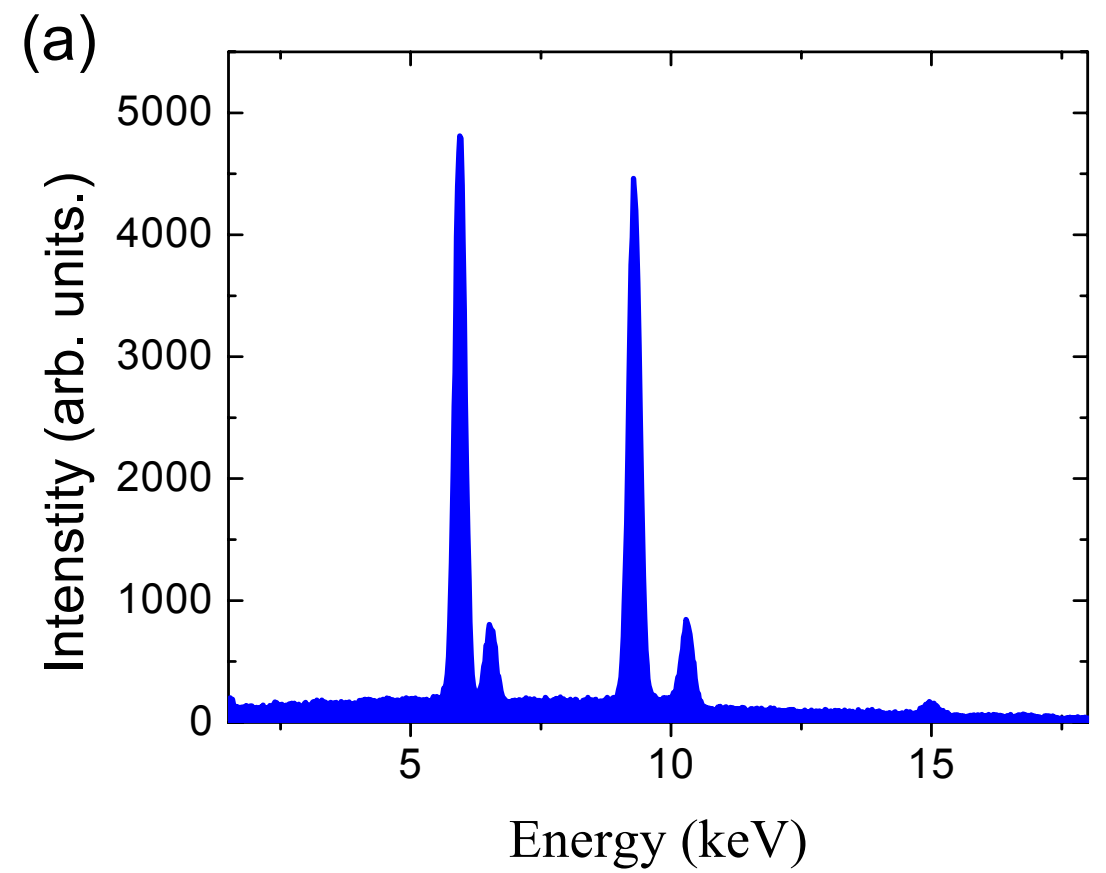

(b)

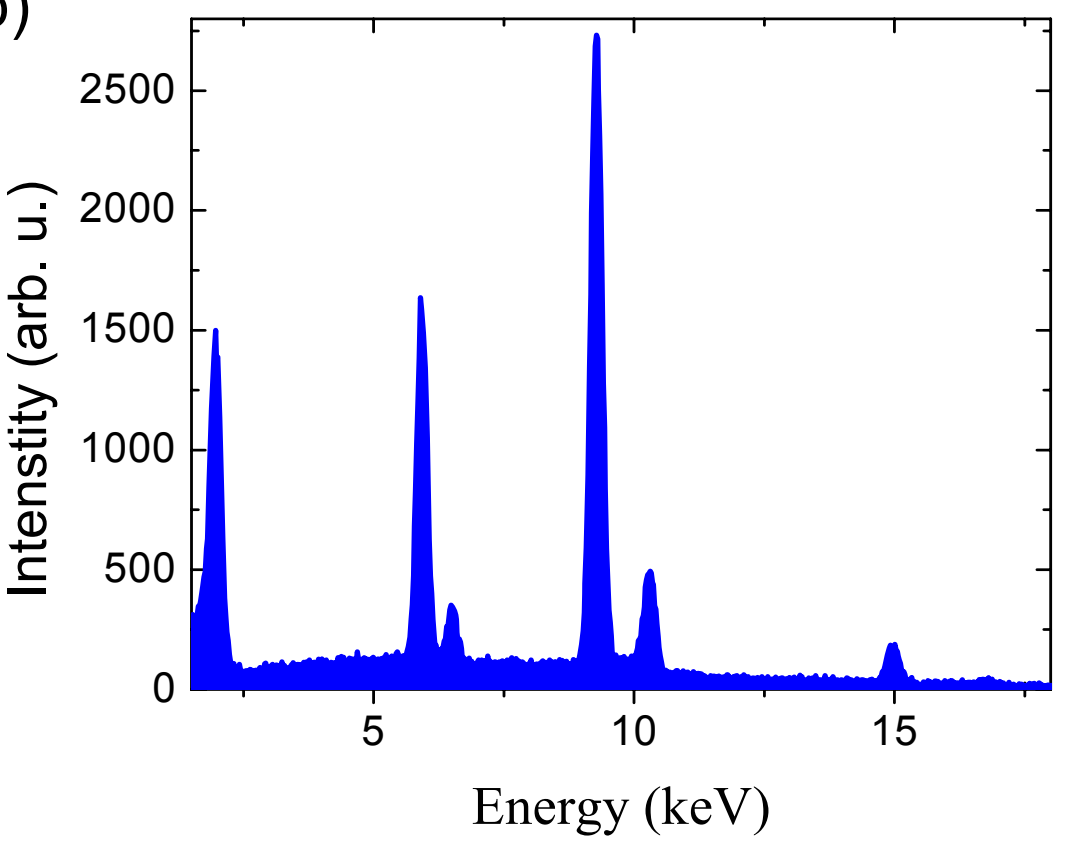

Figure 18

The energy dispersive spectrum for Mn on Y (a) and Y on Mn (b) samples at angle of 20 mrad. Clearly visible are the $\mathrm{Mn}$ and $\mathrm{Ga} \mathrm{K}_{\alpha}, \mathrm{K}_{\beta}$ peaks and $\mathrm{Y} \mathrm{K}_{\alpha}$ peak. The peak at 1.5 $\mathrm{keV}$ in figure (b) is the $\mathrm{Al} \mathrm{K}_{\alpha}$ peak from the sapphire template. 

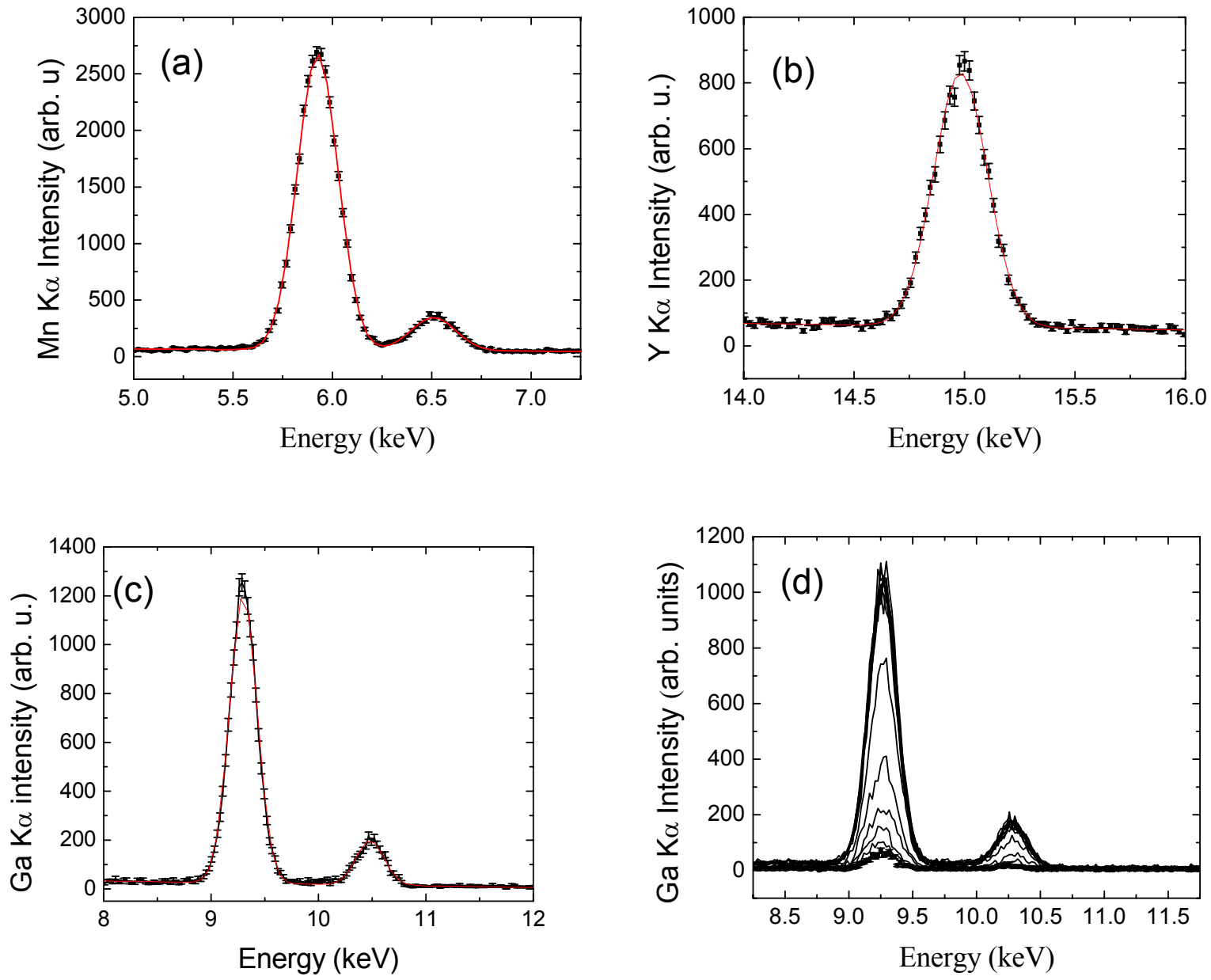

\section{Figure 19}

The sectioned-off portion of EDS showing the characteristic peak and the best fit using a Gaussian function and a linear background for (a) Mn, (b) Y and (c) GaN. (d) shows the variation in $\mathrm{Ga} \mathrm{K}_{\alpha}$ intensity at various take-off angles. 


\subsubsection{X-ray reflectivity}

X-ray reflectivity (XRR) is a well established, non-destructive technique used for estimation of thickness and roughness of thin film structures and was used to verify the structural estimates obtained from RHEED-TRAXS experiments.

The technique involves measuring the reflected X-ray intensity as a function of incidence angle over a range of angles close to the critical angle for total reflection. Above this critical angle the specularly reflected intensity decreases with a form that is dependent on the structural properties of the structure. A typical reflectivity curve is essentially a combination of the Fresnel reflectivity and an interference pattern (Kiessig fringes) from the scattering at different layers (for example, in a thin film or multilayer). If the profile of the sample is known, good estimations of the layer thickness and roughness values can be made by analyzing the reflectivity curve.

The measurements were carried out using a two-axis goniometer with a $\mathrm{Cu}$ rotating anode as a source. A bent-crystal graphite monochromator was used to select the $\mathrm{Cu}$ $\mathrm{K}_{\alpha}$ radiation and to focus the beam in the vertical direction at the sample position. Horizontal slits were used to bring the resolution of the instrument to approximately $0.003 \AA^{-1}$. Prior to each scan, a $2 \theta$ scan was done to align the beam and observe the profile of the incident beam. The sample was then mounted on the goniometer and a rocking curve measurement was performed to assure that the sample is at the center of rotation. After that, $\theta-2 \theta$ scans were performed through an angle range around the specular condition such that $\mathrm{Q}$, the change in $\mathrm{x}$-ray wave vector, is perpendicular to the surface. Typical $\theta-2 \theta$ scans were done between $0.2^{\circ}$ and $7^{\circ}$. 
The same measurements were also run for off-specular alignment of the sample and the data was subtracted from the specular scans as background. The true specular reflectivity curve obtained was normalized to the maximum and analyzed using the software Parratt32 [42]. Parratt32 can handle multiple layered structures. It's based on Parratt formalism for analysis and uses one dimensional Newton-Raphson method for fitting the data. The results from the analysis are discussed further on.

\subsection{X-ray Photoelectron Spectroscopy (XPS)}

The technique of XPS, also known as electron spectroscopy for chemical analysis (ESCA) was developed by K. Siegbahn and his research group in the 1960s. It's a surface sensitive technique that can be used for elemental and chemical analysis, such as type of bonding and chemical states. It probes approximately top $10 \mathrm{~nm}$ of a material and has an accuracy of $10 \%-20 \%$. It can detect elements with atomic number 3 and above with an average detection level of $0.1-0.5$ atomic $\%$. It is mostly non-destructive except when used for concentration profiling. During concentration profiling, a few thousand angstroms of the material can be etched away to reveal the bulk characteristics [43].

The analysis is based on the photoelectric effect and x-rays are used to knock out electrons from the inner shell. The surface of the sample is bombarded by a monochromatic X-ray beam (usually $\mathrm{Al} \mathrm{K}_{\alpha}$ at $1486.6 \mathrm{eV}$ ) and the ejected electrons are filtered based on their kinetic energy. The binding energy, $E_{\text {binding }}$ of the atoms can be calculated because the energy $E_{h v}$ of the exciting radiation is known, so that

$$
E_{\text {binding }}=E_{\text {kinetic }}-E_{h v}-\varphi_{\text {spec }} \text {, }
$$

where $\varphi_{\text {spec }}$ is the work function of the spectrometer. 
The XPS spectrum is described by a succession of peaks which correspond to a given $E_{\text {binding }}$ (for example, $\mathrm{C} 1 \mathrm{~s}$ peak is the excitation of carbon 1s level electrons), that's why it is possible to perform elemental analyses. Also, the signal under each peak of element $\mathrm{A}$ is proportional to the number of $\mathrm{A}$ atoms, so the analysis can be quantitative.

The sampling depth of XPS depends on the mean free path of the electrons coming out of the material. About $70 \%$ of the detected signal comes from the first $1 \AA$, beyond 3 mean free paths, which is $50-100 \AA$, the signal contribution is almost negligible.

The experiments were performed here at West Virginia University in the XPS chamber by ULVAC-PHI Inc. (model PHI5000-versaprobe). The essential features of the instrument are a hemispherical analyzer for guiding ejected electrons and a monochromatic x-ray source as the energy of the ejected electrons depends on that. The electrons ejected from the sample are directed into the hemispherical analyzer entrance slit and electrostatic field within the analyzer allows the electrons with certain energy to pass through and fall on a position sensitive detector that produces a kinetic energy dispersive spectrum of the electrons detected. 


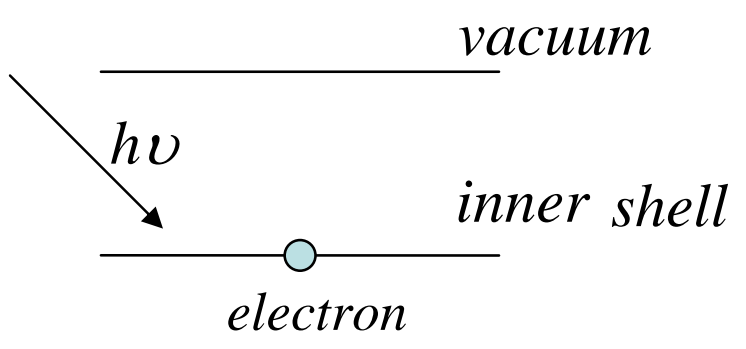

(a)Initial State

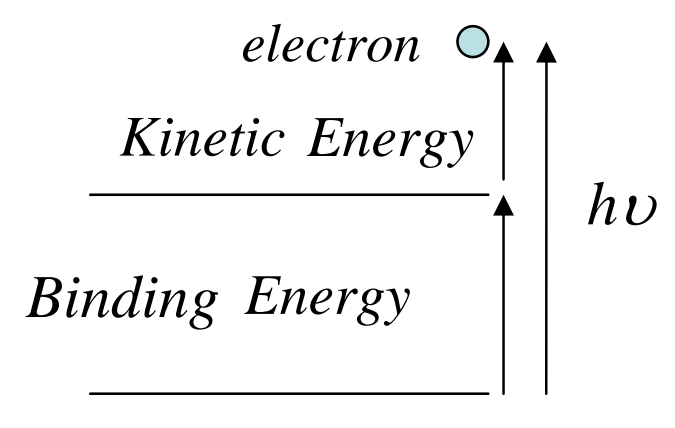

(b)Final State

\section{Figure 20}

The estimation of the binding energy of an electron using monochromatic radiation and the kinetic energy based filtering of the ejected electrons in XPS experiment. XPS can be used for quantitative compositional analysis and determining the oxidation states of the elements. 


\subsection{Rutherford backscattering (RBS)}

RBS is a non-destructive ion scattering technique that is used for thin compositional analysis. RBS allows quantification without the use of standard and is frequently used to calibrate other analytical methods. It can also produce a depth profile of the relative compositions without sputtering the film, for a depth of up to 1 micron.

During RBS experiment, high energy alpha particles $(\mathrm{He}++)$ are directed at the sample and the energy distribution and yield of the backscattered particles is recorded. The energy of the particles depend of the atoms from which they scatter and the depth at which the collision takes place. Since the backscattering cross section for all the elements is known, the relative intensities of the backscattered yield for the elements scaled by their cross sections gives an estimate of their relative compositions, at any depth in the film [44]. RBS was used to verify the results obtained from RHEED-TRAXS for compositional analysis. 


\section{Structural Analysis}

The dependence of the fluorescent signal on the take-off angle and the amount of material deposited was investigated in the initial experiments. No collimating slit was used for the initial experiments. The angular dependence showed a characteristic peak at the critical angle and the strength of the signal was found proportional to the amount of material deposited. The relationship between the signal and the material deposited was not strictly linear because the contribution from x-rays coming from deeper in the material was not same as that from the top layer. So the signal increases linearly for a few nanometers and then starts to flatten out, approaching a bulk-like behavior which occurs when adding more material doesn't cause any difference in the spectrum. Figure 21(a) shows the dependence of the fluorescent signal on the thickness of Mn deposited for the data collected at $9 \mathrm{mrad}$ angle. Figure 21(b) shows the angular dependence of $\mathrm{Mn}$ for various thicknesses of the material. The increase in the signal intensity and a well-defined critical angle peak is clearly visible. A similar angular dependence can be seen in the Y peak of

Y thin films with the critical angle $\sim \sqrt{2 \delta_{2}}$. The peak areas were calculated by fitting the elemental peaks to a Gaussian curve over a linear background.

To verify the simulation results, single and bilayer samples of $\mathrm{Y}$ and $\mathrm{Mn}$ were grown on a GaN on sapphire substrate and then capped with a layer of aluminum. This preserved the thickness of $\mathrm{Mn}$ and $\mathrm{Y}$ layers under $\mathrm{Al}$ by preventing them from being oxidized once samples were removed from vacuum, as Al forms a thin oxide layer on top that prevents further oxidation of the material. This was a very important step as the RHEED-TRAXS results had to be verified using x-ray reflectivity measurement and both 

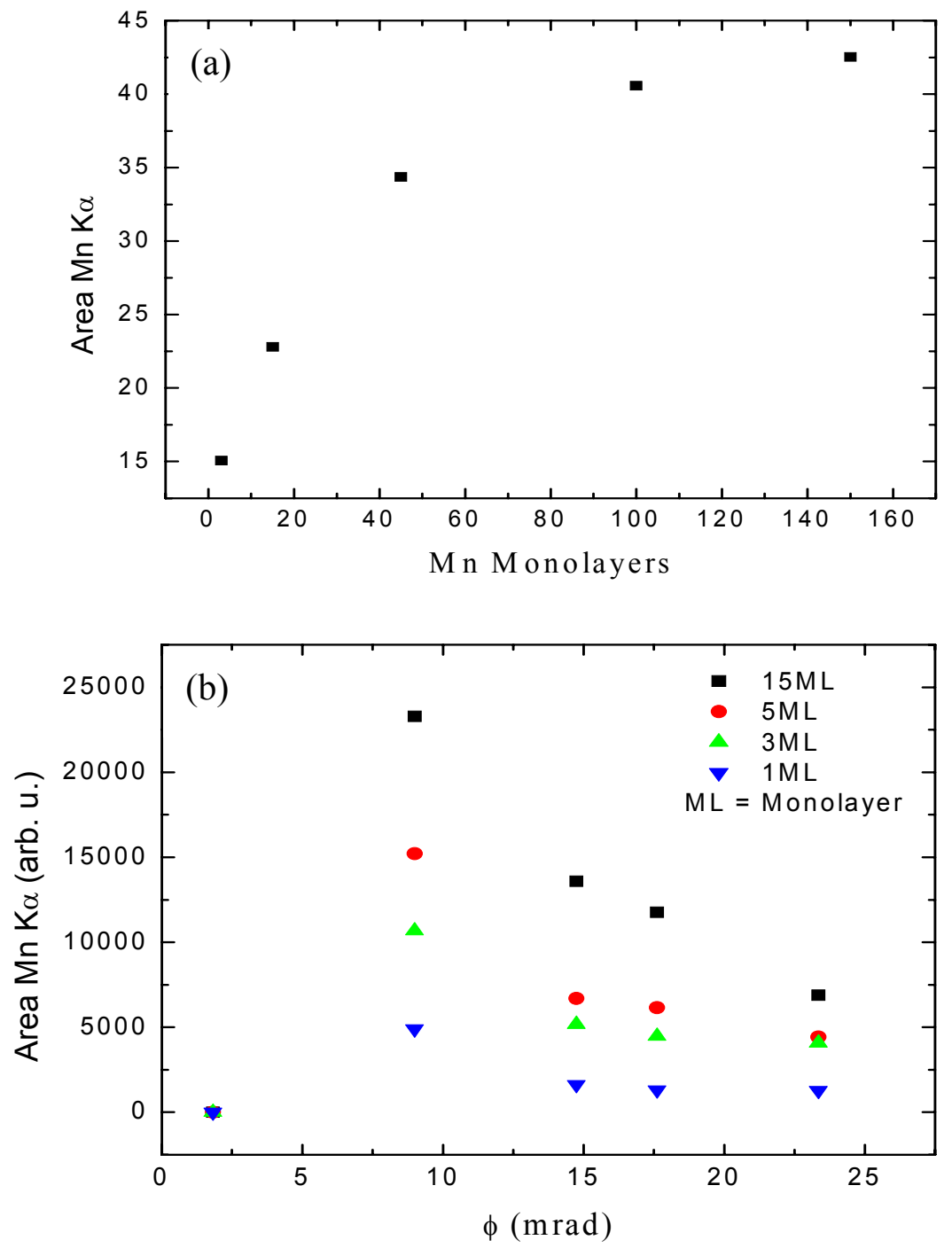

\section{Figure 21}

(a) The area under $\mathrm{Mn} \mathrm{K}_{\alpha}$ peak at the grazing angle of $9 \mathrm{mrad}$. The linear trend for small thickness flattens out as the thickness is increased, signifying that the contribution from deeper layers has diminishing effect on the intensity. No collimating slit was used. 1 monolayer of $\mathrm{Mn}$ is 2.3 angstrom thick.

(b)The angular dependence of $\mathrm{Mn} \mathrm{K}_{\alpha}$ peaks for varying thickness during initial experiments. The thickness estimate is based on crystal monitor calibration of Mn flux. No collimating slit was used. 
$\mathrm{Mn}$ and $\mathrm{Y}$ are susceptible to oxidation.

Before depositing the materials, the manipulator was set to a position, in height and rotation, that should remain the same for the entire experiment as the angular dependence data for the Ga $\mathrm{K}_{\alpha}$ peak were collected. The data were later used to calibrate the angle for the $\mathrm{Mn}$ and $\mathrm{Y}$ angular dependence data analysis.

For a single layer of Mn and $\mathrm{Y}$, the electron trajectory simulation using CASINO was performed once and the results for the depth profile were stored. The reason for doing this was that,to the in the first approximation, the thickness of the layer did not affect the x-ray depth profile. The effect of the Ga x-rays from the substrate exciting Mn atoms on top and adding to the fluorescence generated by the incident electrons was ignored. The secondary effects of an underlayer, such as a Y layer under Mn, were also ignored. But while analyzing the fluorescence signal from a buried layer, the effect of number of electrons with sufficient ionizing energy reaching the layer depended on the thickness of the overlayer(s). So, for analyzing the signal from an underlayer, a CASINO simulation was carried out initially with a sufficiently thick overlayer(s) to reach at the first estimate for the layer thickness value(s) using the routine for structural analysis. The thickness estimates thus obtained were then used to run the CASINO simulation again and the x-ray profile in the layer of interest obtained was plugged back into the same routine for structural analysis. This recursive process was carried out until agreeing values were obtained from two successive structural fitting routines. The results showed that the x-ray profiles were not very sensitive to the overlayer thickness values and a convergence was reached in two to three iterations. The thickness of the overlayer mostly changed the magnitude of the x-rays produced, but the change in the shape was not 
significant enough to produce a considerable change in the overall angular dependence of the x-rays. With good initial estimates for the overlayers, the multiple CASINO simulation process can be avoided. The following sub-sections discuss the results from various layered structures deposited on $\mathrm{GaN}$ substrates.

\subsection{Mn on GaN}

Approximately $25 \mathrm{~nm}$ of Mn, based on a quartz crystal monitor (QCM) calibration, was deposited on $\mathrm{GaN}$ and capped with an $\mathrm{Al}$ layer. RHEED-TRAXS experiments were performed after every layer deposition and prior to that for calibration purposes (Figure 22(c)). The RHEED-TRAXS measurements shown in Figure 22(a) yielded the thickness of $23 \pm 3 \mathrm{~nm}$ and a roughness of $1 \pm 1 \mathrm{~nm}$ for $\mathrm{Mn}$ for a single layer of $\mathrm{Mn}$. The roughness value for the GaN underlayer showed the error estimates outside the range analyzable using the roughness model, indicating a low sensitivity for the parameter. So the roughness parameter for GaN surface was set to zero and kept constant during the fitting

process. After the deposition of $\mathrm{Al}$ layer, the measurement on the $\mathrm{Al} / \mathrm{Mn}$ structure yielded the thickness values of $8.3 \pm 0.4 \mathrm{~nm}$ and $25 \pm 2 \mathrm{~nm}$ for $\mathrm{Al}$ and $\mathrm{Mn}$, respectively. The roughness value for $\mathrm{Mn}$ was $1 \pm 1 \mathrm{~nm}$ again. The roughness values for the $\mathrm{Al}$ overlayer was set to zero and kept constant due to the aforementioned reason.

Once the sample was removed from the system, x-ray reflectivity (XRR) measurements were performed to verify the results. XRR estimates, based on the scans shown in Figure 22(b), were $10 \pm 2 \mathrm{~nm}$ and $26.1 \pm 0.5 \mathrm{~nm}$ for thickness values of $\mathrm{Al}$ and Mn layers, respectively, and $0.2 \pm 0.1 \mathrm{~nm}$ for Mn roughness. Hence, the XRR results agreed with the RHEED-TRAXS results to within their uncertainty. All the results are summarized in Table 1. 

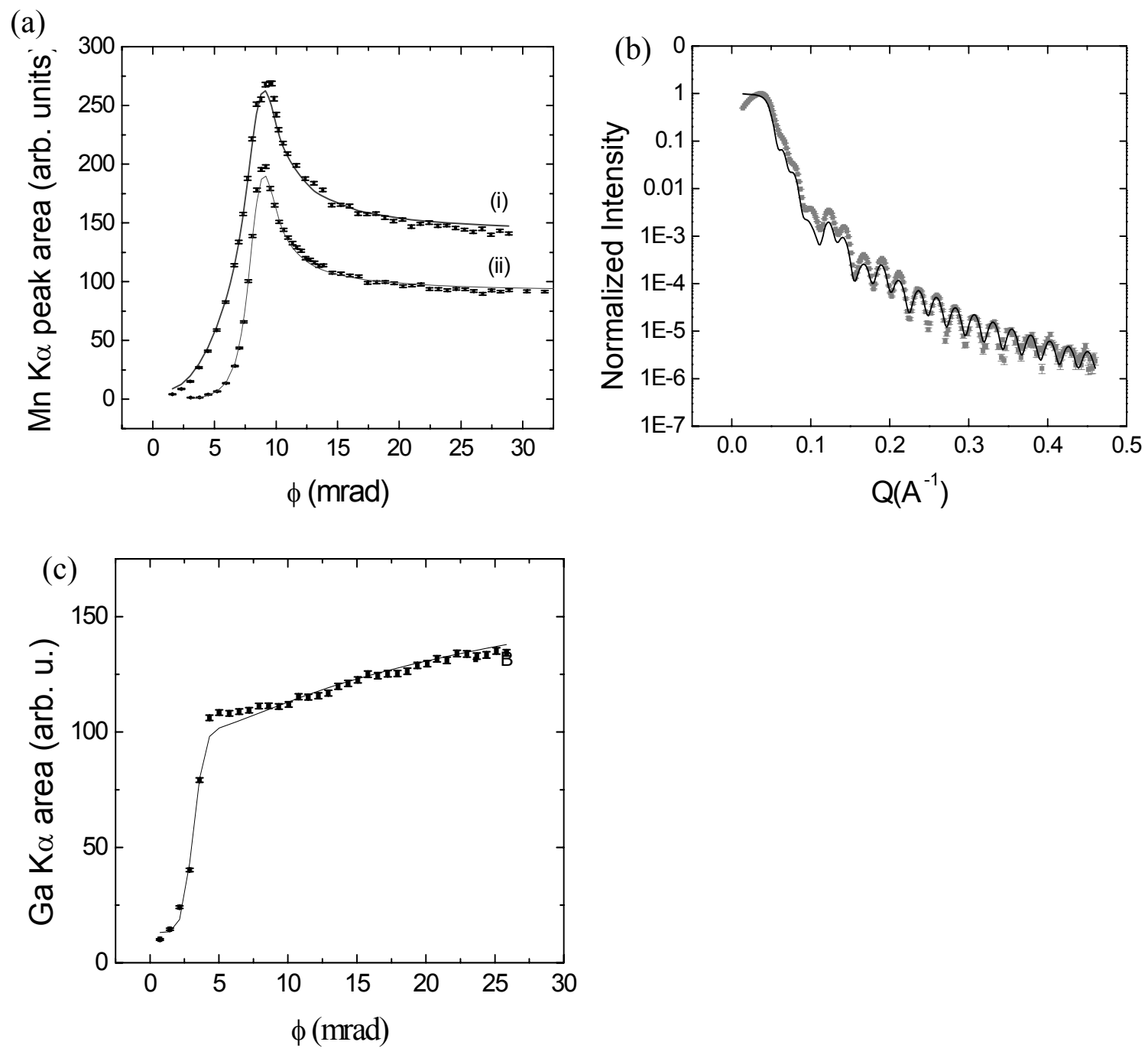

\section{Figure 22}

(a) RHEED-TRAXS measurements and (b) XRR measurements on a Mn film sample.

The symbols are the data and the solid curves are the non-linear fits to the data. (i) and (ii) correspond to data obtained prior and after the Al deposition, respectively. The parameters used in this fit are shown in Table 1. (c) The angular dependence of Ga from GaN substrate that was used to calibrate the angle. 


\section{Table 1}

Structural parameters for the GaN/Mn/Al sample obtained from x-ray reflectivity (XRR) and RHEED-TRAXS. The thickness of the layer is $d$ and the interfacial roughness is $\sigma$. The uncertainties in the values were obtained from the non-linear least squares fit procedure. The Mn_GaN data were obtained after the Mn deposition and the Al_Mn_GaN profile was obtained after the Al layer deposition. The characteristic x-ray peak used for the data analysis is indicated.

\begin{tabular}{|c|c|c|c|c|c|c|}
\hline \multicolumn{2}{|c|}{ Layer Material } & \multicolumn{2}{|c|}{ Al } & \multicolumn{2}{|c|}{ Mn } & \multirow{2}{*}{$\frac{\text { GaN }}{\sigma(\mathrm{nm})}$} \\
\hline & & d (nm) & $\sigma(\mathbf{n m})$ & d (nm) & $\sigma(\mathbf{n m})$ & \\
\hline \multicolumn{2}{|c|}{ XRR } & $10 \pm 2$ & $0.9 \pm 0.9$ & $26.1 \pm 0.5$ & $0.2 \pm 0.1$ & $0.2 \pm 0.2$ \\
\hline \multicolumn{7}{|c|}{ RHEED-TRAXS } \\
\hline Profile & Peak & & & & & \\
\hline Mn_GaN & Mn K $\alpha$ & -- & -- & $23 \pm 3$ & $1 \pm 1$ & -- \\
\hline Al_Mn_GaN & Mn Ka & $8.3 \pm 0.4$ & - & $25 \pm 2$ & $1 \pm 1$ & -- \\
\hline
\end{tabular}




\subsection{Y on GaN}

The same experiment, as described in the previous section, was performed with approximately $20 \mathrm{~nm}$ thick layer of Y deposited on Mn. RHEED-TRAXS data (Figure 23(a)) yielded the results of $19 \pm 2 \mathrm{~nm}$ and $1.9 \pm 0.4 \mathrm{~nm}$ for thickness and roughness values for a single layer. For $\mathrm{Al} / \mathrm{Y}$ structure, the thickness values for $\mathrm{Al}$ and $\mathrm{Y}$ were $15 \pm 8 \mathrm{~nm}$ and $13 \pm 7$, respectively. The roughness for $Y$ layer was $5 \pm 2 \mathrm{~nm}$. In comparison with this, the ex-situ XRR measurements yielded the thickness of $8.6 \pm 0.8 \mathrm{~nm}$ and $18 \pm 1 \mathrm{~nm}$ for $\mathrm{Al}$ and $\mathrm{Y}$, respectively. The roughness estimate for $\mathrm{Y}$ layer was $0.3 \pm 0.4 \mathrm{~nm}$.

Comparing these results with the results from the previous section, it was obvious that the estimates became much poorer when Al layer was deposited on top. This was because of the reduced signal quality and also the relative sensitivity of $\mathrm{Y}$ and Mn X-rays to the $\mathrm{Al}$ overlayer parameters.

The fluorescent yield, i.e., the probability that an atom will relax by the emission of a photon, is 0.72 for $\mathrm{Y}$ as compared with 0.30 for Mn. But the $\mathrm{Y} \mathrm{K \alpha}$ peak at $14.96 \mathrm{keV}$ is far less efficiently excited by electron beam of $25 \mathrm{keV}$ as compared to the $\mathrm{Mn} \mathrm{K} \alpha$ peak at $5.9 \mathrm{keV}$ as the ionization cross section is dependent on the over-voltage ratio $\left(\mathrm{E}_{\text {Excitation }} / \mathrm{E}_{\text {critical }}\right)$.Moreover, the efficiency of the detector for $\mathrm{Y} \mathrm{K}_{\alpha}$ peak at $14.96 \mathrm{keV}$ is also smaller than for Mn peak at $5.9 \mathrm{keV}$ (Figure 13). The combined affect of these factors yielded a much smaller signal for $Y$. The signal was even harder to detect when $\mathrm{Y}$ layer was buried under another material.

The other factor that caused the poorer estimates for the sample with Al layer over $\mathrm{Y}$ was the difference in the critical angles for $\mathrm{Mn}$ and $\mathrm{Y}$. The Al layer acted as a rarified medium and caused the x-rays to bend towards the normal to the surface, causing the 
sharpening of the peak at the critical angle. Since the effect of an Al overlayer was most prominent around the critical angle, Mn, with critical angle at $9 \mathrm{mrad}$, had a greater sensitivity to its parameters during the fitting process as compared to $\mathrm{Y}$, with a critical angle at $3 \mathrm{mrad}$ (see Eqs. 18 and 21). Hence, Al overlayer thickness determination using Mn yielded better results, in general. The results are summarized in Table 2. 
(a)
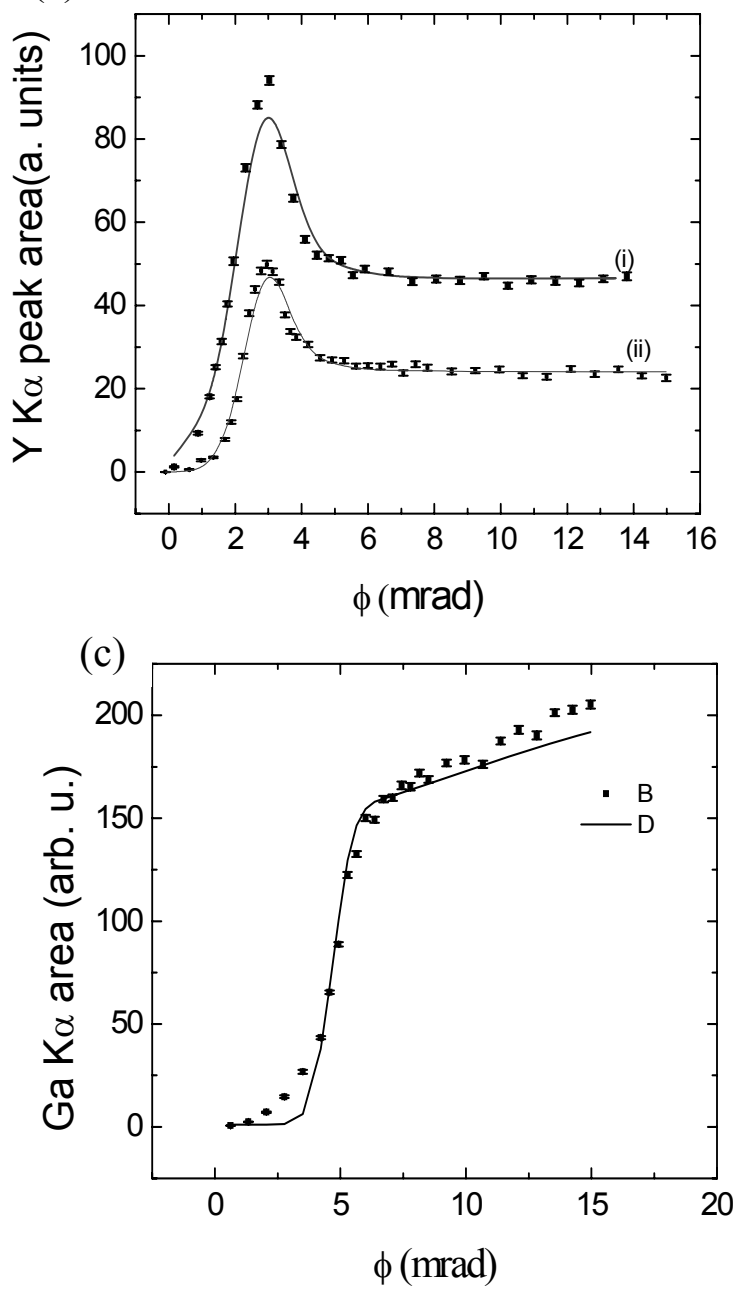

(b)

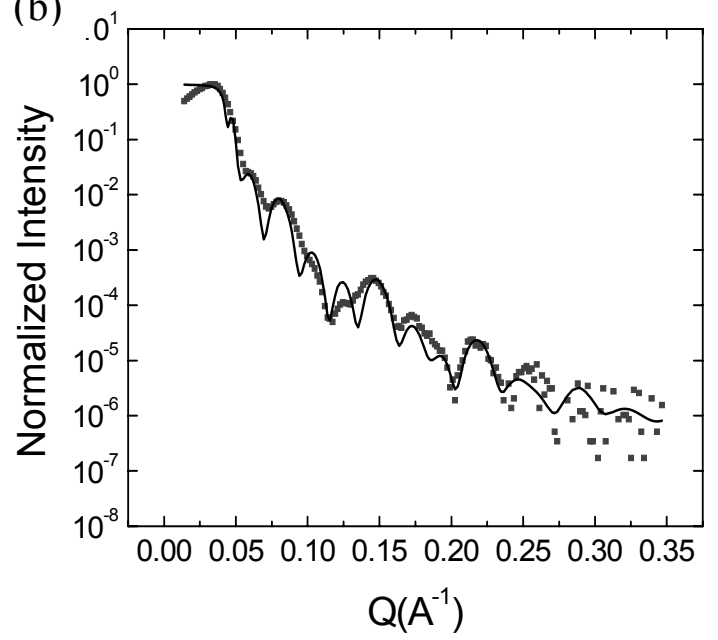

Figure 23

(a) RHEED-TRAXS measurements and (b) XRR measurements on a Y film sample. The symbols are the data and the solid curves are the non-linear fits to the data. (i) and (ii) correspond to data obtained prior and after the Al deposition, respectively. The parameters used in this fit are shown in Table 1. (c) The angular dependence of Ga from GaN substrate that was used to calibrate the angle. 


\section{Table 2}

Structural parameters for the GaN/Y/Al sample obtained from X-ray reflectivity (XRR) and RHEED-TRAXS. The thickness of the layer is $d$ and the interfacial roughness is $\sigma$. The uncertainties in the values were obtained from the non-linear least squares fit procedure. The Y_GaN data were obtained after the $\mathrm{Y}$ deposition and the Al_Y_GaN profile was obtained after the Al layer deposition. The characteristic x-ray peak used for the data analysis is indicated.

\begin{tabular}{|c|c|c|c|c|c|c|}
\hline \multicolumn{2}{|c|}{ Layer Material } & \multicolumn{2}{|c|}{ Al } & \multicolumn{2}{|c|}{$\mathbf{Y}$} & \multirow{2}{*}{$\frac{\mathrm{GaN}}{\sigma(\mathrm{nm})}$} \\
\hline & & d (nm) & $\sigma(\mathrm{nm})$ & d (nm) & $\sigma(\mathrm{nm})$ & \\
\hline XR & & $8.6 \pm 0.8$ & $0.8 \pm 0.4$ & $18 \pm 1$ & $0.3 \pm 0.4$ & $0.7 \pm 0.4$ \\
\hline \multicolumn{7}{|c|}{$\begin{array}{c}\text { RHEED-TRAXS } \\
\text { results }\end{array}$} \\
\hline Profile & Peak & & & & & \\
\hline Y_GaN & Y K $\alpha$ & -- & -- & $19 \pm 2$ & $1.9 \pm 0.4$ & -- \\
\hline Al_Y GaN & $\mathbf{Y} \mathbf{K} \alpha$ & $15 \pm 8$ & -- & $13 \pm 7$ & $5 \pm 2$ & -- \\
\hline
\end{tabular}




\subsection{Y/Mn bilayer on GaN}

A layered structure of $\mathrm{Y}$ on $\mathrm{Mn}$ was grown on GaN substrate and capped with $\mathrm{Al}$. In this case, there were two analyzable peaks in the energy dispersive spectrum coming from different layers in the structure. As before, RHEED-TRAXS measurements were performed before and after every layer deposition and the peaks from the buried layers of Mn and Y were analyzed as well (Figure 24 (a) \& (b)). The thickness estimates from Mn were in agreement with the XRR results even when the layer was buried under $\mathrm{Y}$ and $\mathrm{Al} / \mathrm{Y}$, as summarized in Table 3 . The roughness values were once again reported for the layer investigated and fall within the error range from ex-situ measurements with increasing uncertainty as the layer got buried under $\mathrm{Y}$ and Al. With Mn signal, the thickness estimate of not just Y layer on top but also the Al layer on top of Y could be obtained.

The Y signal yielded information about the Y layer but the angular dependence was not sensitive to the thickness of the Mn layer at the bottom. The fitting process yielded unacceptable error estimates for the Mn layer thickness. So the Mn thickness was held constant during the fitting. When Y got buried under Al layer, the roughness of the layer could not be estimated, but it yielded the thickness values of the $\mathrm{Y}$ and $\mathrm{Al}$ overlayers within the ex-situ estimates.

The most remarkable observation while analyzing this multilayered structure was the insensitivity of Y fluorescence signal to the thickness of the Mn underlayer. This can be understood by realizing that the variation in the underlayer parameters, such as thickness, was reflected to a greater degree in $E_{n}^{R}$, the part of the wave reflected from the bottom of the investigated layer. Since the contribution of $E_{n}^{R}$ towards the total intensity 
in much smaller than that of $E_{n}$, a variation in the underlayer parameter was harder to detect by fitting the angular dependence of the total intensity. 
(a)

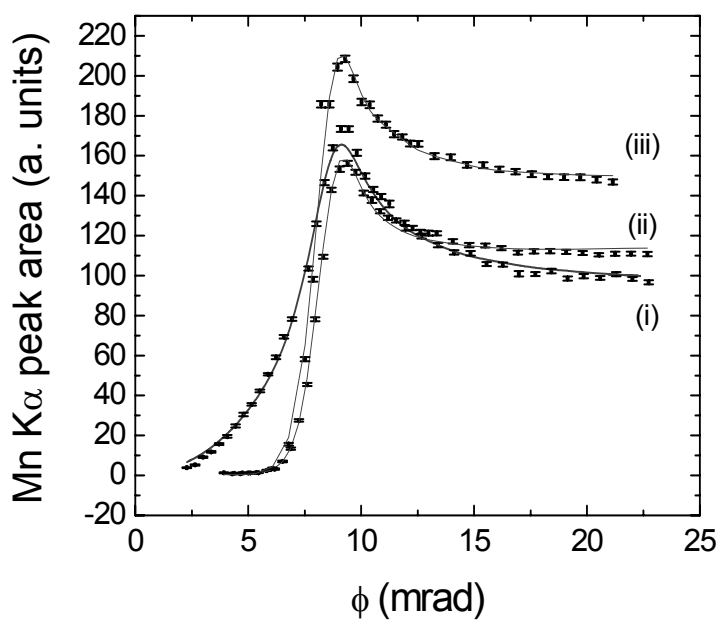

(c)

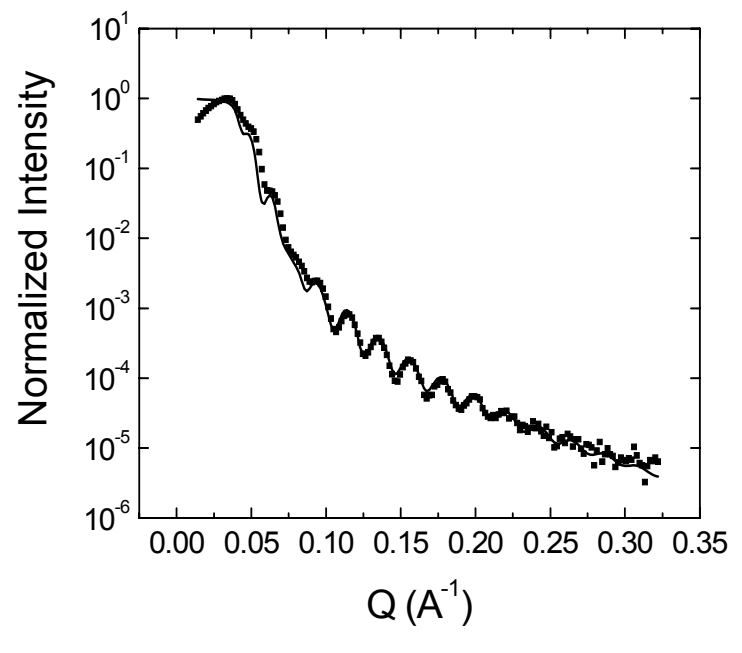

(b)

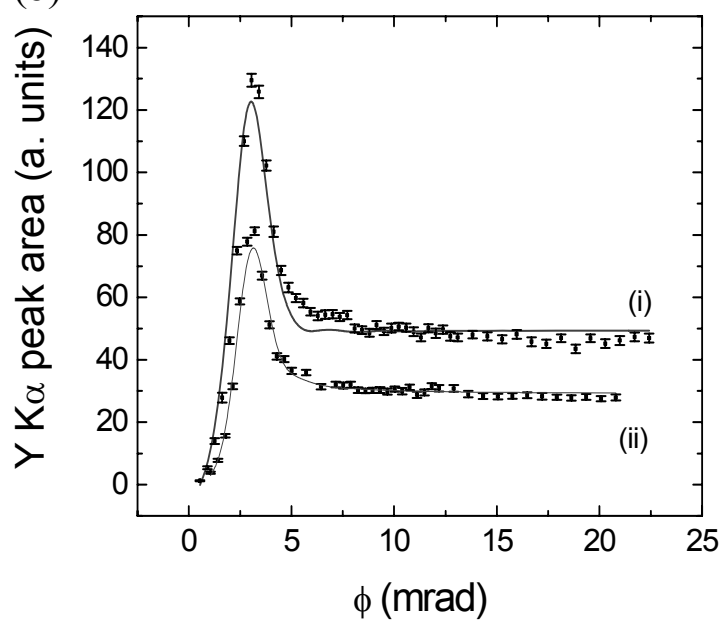

(d)

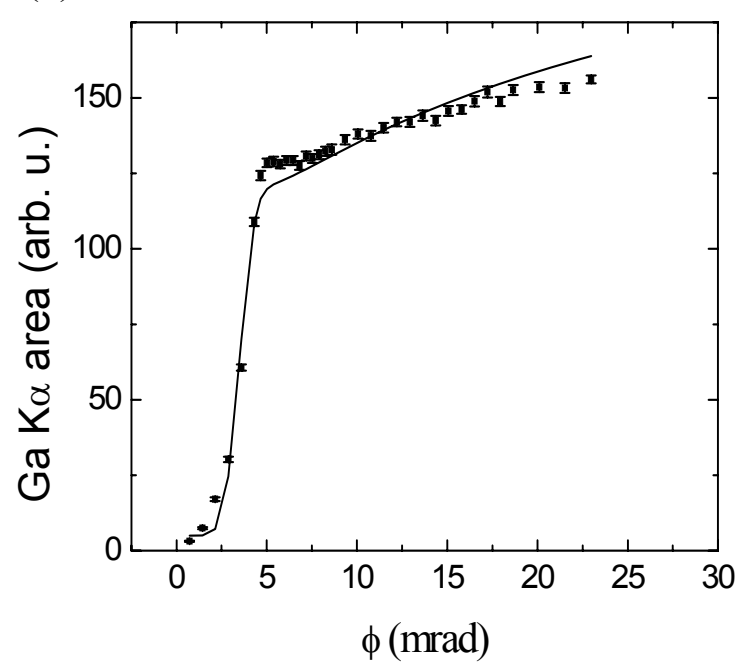

Figure 24

RHEED-TRAXS and measurements using (a) Mn $\mathrm{K}_{\alpha}$ fluorescence and (b) $\mathrm{Y} \mathrm{K}_{\alpha}$

fluorescence on the GaN/Mn/Y/Al sample. In (b), (i), (ii) and (iii) are data obtained after deposition of the Mn layer, the Y layer and the Al layer, respectively. Symbols represent the data, solid curves non-linear fits to the data. In (b), (i) and (ii) are data obtained after the deposition of Y layer and the Al layer. The parameters used for the fits are shown in Table 3. (c) X-ray reflectivity measurements. (d) Ga angular dependence for angular calibration. 
Table 3

Structural parameters for the GaN/Mn/Y/Al sample obtained from x-ray reflectivity (XRR) and RHEED-TRAXS. The thickness of the layer is $d$ and the interfacial roughness is $\sigma$. The uncertainties in the values were obtained from the non-linear least squares fit procedure. The Mn_GaN, Y_Mn_GaN, Al_Y_Mn_GaN data were obtained after the $\mathrm{Mn}, \mathrm{Y}$, and $\mathrm{Al}$ depositions, respectively. The characteristic x-ray peak used for the data analysis is indicated.

\begin{tabular}{|c|c|c|c|c|c|c|c|c|}
\hline \multicolumn{2}{|c|}{ Layer Material } & \multicolumn{2}{|c|}{ Al } & \multicolumn{2}{|c|}{$\mathbf{Y}$} & \multicolumn{2}{|c|}{ Mn } & \multirow{2}{*}{$\frac{\mathrm{GaN}}{\mathrm{\sigma}(\mathrm{nm})}$} \\
\hline & & $d(\mathrm{~nm})$ & $\sigma(\mathbf{n m})$ & $d(\mathrm{~nm})$ & $\sigma(\mathrm{nm})$ & $d(\mathrm{~nm})$ & $\sigma(\mathbf{n m})$ & \\
\hline \multicolumn{2}{|l|}{ XRR } & $12 \pm 3$ & $2 \pm 1$ & $12 \pm 5$ & $3 \pm 2$ & $28 \pm 2$ & $0.2 \pm 0.9$ & $0.8 \pm 0.2$ \\
\hline \multicolumn{9}{|c|}{ RHEED-TRAXS } \\
\hline Profile & Peak & & & & & & & \\
\hline Mn_GaN & Mn Ko & -- & -- & -- & -- & $29 \pm 4$ & $1 \pm 2$ & -- \\
\hline Y_Mn_GaN & $\mathbf{Y} \mathbf{K} \alpha$ & - & -- & $15 \pm 1$ & $3 \pm 0.4$ & 28 * & -- & -- \\
\hline Y_Mn_GaN & Mn Ko & -- & -- & $14.6 \pm 0.4$ & $1.3 \pm 0.3$ & $28 \pm 2$ & $0 \pm 3$ & -- \\
\hline Al_Y_Mn_GaN & $\mathbf{Y} \mathbf{K} \alpha$ & $9 \pm 2$ & -- & $8.4 \pm 0.9$ & -- & 28 * & -- & -- \\
\hline Al_Y_Mn_GaN & Mn Ko & $15 \pm 4$ & -- & $14 \pm 2$ & -- & $29 \pm 5$ & $1 \pm 4$ & -- \\
\hline
\end{tabular}

*The parameter was held constant during the fitting process. 


\section{$4.4 \mathrm{Mn} / \mathrm{Y}$ bilayer on GaN}

After exploring the single layers of $\mathrm{Y}$ and $\mathrm{Mn}$ and a bilayer sample with two elements, the next logical step was to explore what would happen if the positions of $\mathrm{Y}$ and $\mathrm{Mn}$ were reversed i.e., $\mathrm{Y}$ was buried under a $\mathrm{Mn}$ layer. So a single $\mathrm{Mn} / \mathrm{Y}$ bilayer was grown and capped with $\mathrm{Al}$ as before.

A single layer of $Y$ yielded the thickness estimate of $25 \pm 2 \mathrm{~nm}$ and roughness of $0 \pm 1$ (Figure 25(a)), both in good agreement with the XRR results. Once Mn was deposited on top of the $\mathrm{Y}$, there was a very dramatic change in $\mathrm{Y}$ angular dependence. The sharp peak at the critical angle completely disappeared and the angular dependence became flat after the critical angle (Figure 25(a)). Remarkably, the simulation results for the given conditions gave the same profile. However, the fitting process revealed that the thickness or the roughness could not be reasonably estimated from such a distribution. The Mn layer fluorescence, on the other hand, showed the regular feature of a well-defined critical angle peak and yielded reasonable Mn thickness and roughness estimates. Further, the sensitivity of Mn fluorescence to Y underlayer thickness was better than what had been seen with the previous bilayer sample. Though the error estimate in the underlayer thickness was large, it could be determined from the fitting process, unlike the case when Mn layer was under Y layer. All the results are summarized in Table 4 .

Once capped with $\mathrm{Al}$, only $\mathrm{Mn}$ signal was large enough to be analyzed. The thickness estimates for $\mathrm{Al}, \mathrm{Mn}$ and $\mathrm{Y}$ layers were $6 \pm 1 \mathrm{~nm}, 16 \pm 1 \mathrm{~nm}$ and $27 \pm 5 \mathrm{~nm}$, respectively. In comparison, XRR results were $9 \pm 5 \mathrm{~nm}, 19 \pm 1 \mathrm{~nm}$ and $25 \pm 2 \mathrm{~nm}$ for $\mathrm{Al}$, $\mathrm{Mn}$ and $\mathrm{Y}$, respectively. $\mathrm{Y}$ signal from under $\mathrm{Mn}$ and $\mathrm{Al}$ was too small to be analyzed. 
(a)

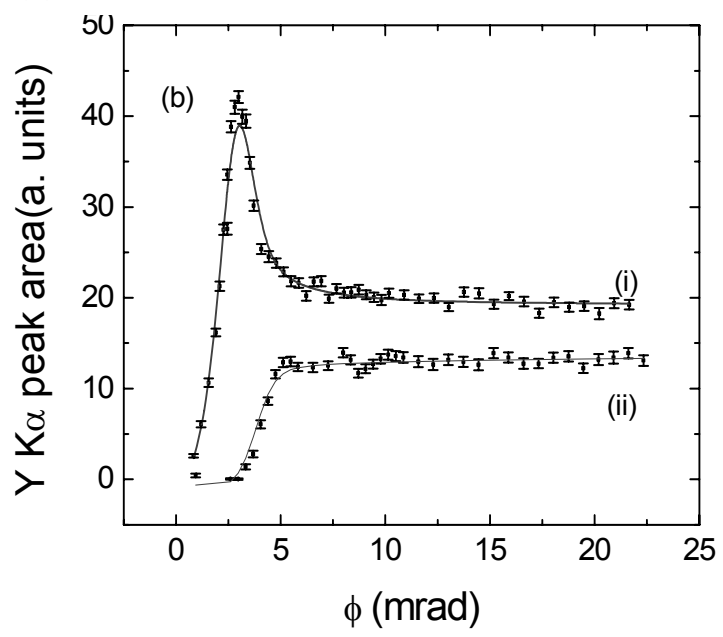

(c)

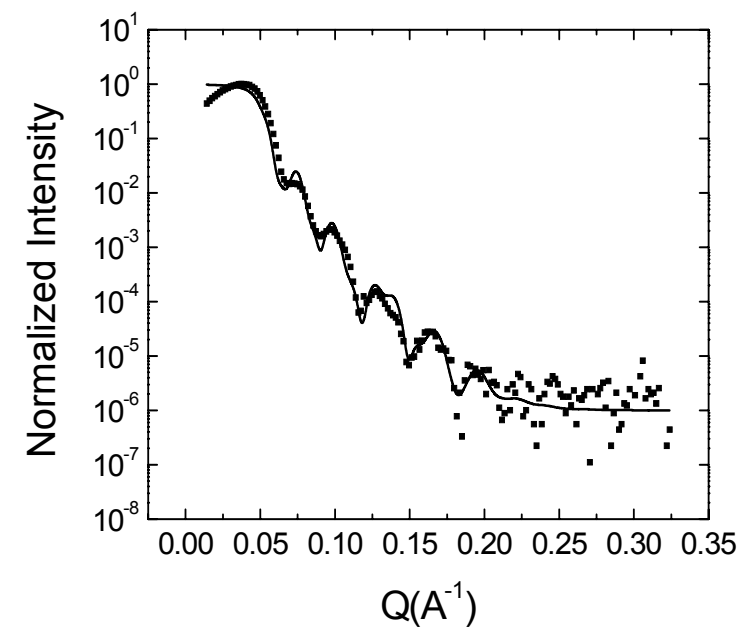

(b)

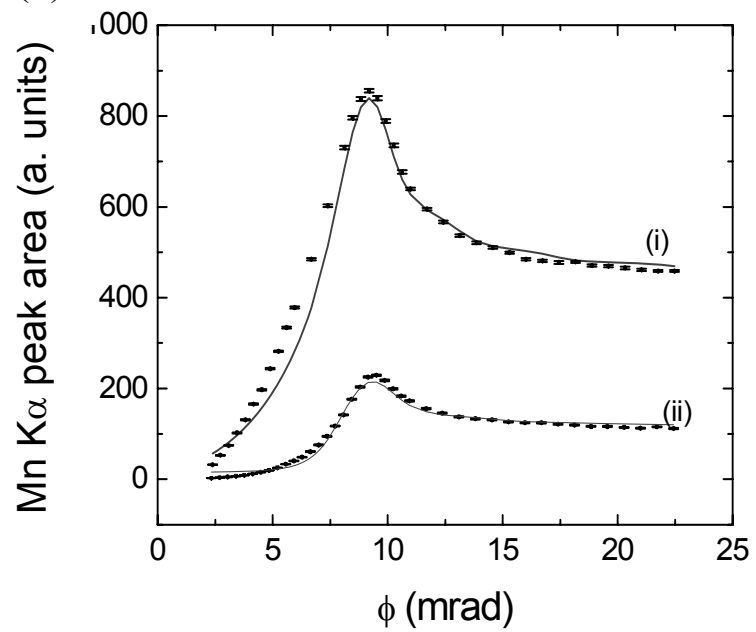

(d)

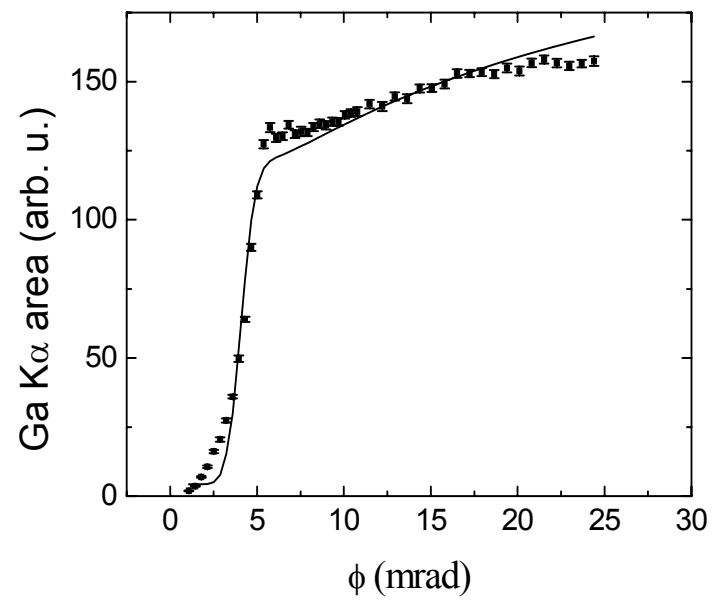

Figure 25

RHEED-TRAXS measurements using (a) $\mathrm{Y} \mathrm{K}_{\alpha}$ fluorescence and (b) $\mathrm{Mn} \mathrm{K}_{\alpha}$ fluorescence on the $\mathrm{GaN} / \mathrm{Y} / \mathrm{Mn} / \mathrm{Al}$ sample. In (a), (i) and (ii) are data obtained after deposition of the Y layer and the Mn layer, respectively. In (b), (i) and (ii) are data obtained after deposition of the Mn layer and the Al layer. Symbols represent the data, solid curves non-linear fits to the data. The parameters used for the fits are shown in Table 4. (c) Xray reflectivity measurements. (d) Ga angular dependence for angular calibration. 
Table 4

Structural parameters for the $\mathrm{GaN} / \mathrm{Y} / \mathrm{Mn} / \mathrm{Al}$ sample obtained from x-ray reflectivity (XRR) and RHEED-TRAXS. The thickness of the layer is $d$ and the interfacial roughness is $\sigma$. The uncertainties in the values were obtained from the non-linear least squares fit procedure. The Y_GaN, Mn_Y_GaN, Al_Mn_Y_GaN data were obtained after the $\mathrm{Y}, \mathrm{Mn}$, and $\mathrm{Al}$ depositions, respectively. The characteristic x-ray peak used for the data analysis is indicated. Y signal from under Al and Mn layers was too weak to be analyzed.

\begin{tabular}{|c|c|c|c|c|c|c|c|c|}
\hline \multicolumn{2}{|c|}{ Layer Material } & \multicolumn{2}{|c|}{ Al } & \multicolumn{2}{|c|}{ Mn } & \multicolumn{2}{|c|}{$\mathbf{Y}$} & \multirow{2}{*}{$\begin{array}{c}\text { GaN } \\
\sigma(\mathrm{nm})\end{array}$} \\
\hline & & D (nm) & $\sigma(\mathrm{nm})$ & $D(\mathbf{n m})$ & $\sigma(\mathrm{nm})$ & d (nm) & $\sigma(\mathrm{nm})$ & \\
\hline \multicolumn{2}{|l|}{ XRR } & $9 \pm 5$ & $1.8 \pm 0.9$ & $19 \pm 1$ & $1.4 \pm 0.5$ & $25 \pm 2$ & $0.9 \pm 0.3$ & $1.2 \pm 0.9$ \\
\hline \multicolumn{9}{|c|}{ RHEED-TRAXS } \\
\hline Profile & Peak & & & & & & & \\
\hline Y_GaN & $\mathbf{Y} \mathbf{K} \alpha$ & -- & -- & -- & -- & $25 \pm 2$ & $\mathbf{0} \pm \mathbf{1}$ & -- \\
\hline Mn_Y_GaN & Mn Ko & -- & -- & $21 \pm 2$ & $1.2 \pm 0.6$ & $23 \pm 9$ & -- & -- \\
\hline Mn_Y_GaN & $\mathbf{Y} \mathbf{K} \alpha$ & -- & -- & $25 \pm 6$ & -- & $22 \pm 21$ & -- & -- \\
\hline AI_Mn_Y_GaN & Mn Ko & $6 \pm 1$ & -- & $16 \pm 1$ & $\mathbf{0} \pm \mathbf{3}$ & $27 \pm 5$ & -- & -- \\
\hline
\end{tabular}




\section{Compositional Analysis}

Compositional analysis is another potential application of RHEED-TRAXS that can be of immense use for in-situ characterization. If performed real time, it could lead to a better stoichiometry control. Initially, the idea behind the compositional analysis was to detect the changes in the angular dependence due to slight changes in the density of the material resulting from the change in relative compositions of the constituent elements. The changes in density and the molar mass of per unit formula can be linked to the refractive index of the material which in turn, defines the shape of the angular dependence in grazing angle regime. The major advantage of adopting this approach was that once again, like structural characterization, there would be no need to do absolute measurements that not only required very precise information about the geometry of the setup but also good standards for calibration.

As it would be described later, the change in the angular dependence due to the change in the refractive index is not large enough to see a change in the angular dependence. However, for a compound, the relative composition of the elements should be proportional to the relative intensity of the fluorescent signal at any given angle. But the absolute intensity of the signal is also related to the total thickness of the material. Also, as the material would have different refractive index for x-rays of different energy, the absorption effects and rate of increase of the fluorescent signal with increasing thickness would be different. Moreover, due to difference in the critical angle and overall shape of the angular dependence, the ratio should change with the angle. This posed a non-trivial problem and required a detailed analysis. 


\subsection{Angular dependence variation with refractive index}

A simulation was performed with a perfectly stiochiometric $\mathrm{YMnO}_{3}$ layer on $\mathrm{GaN}$ and the theoretical curves for the angular dependences for $\mathrm{Y}$ and $\mathrm{Mn}$ were produced. Next, slight variation in the concentrations of $\mathrm{Y}$ and $\mathrm{Mn}$ were analyzed. The variation in composition was accounted for by using Equations 40 and 41 described in section 2.4.

$$
\begin{gathered}
\delta=4.1516 \times 10^{-4} \frac{\rho}{M \cdot E^{2}} \sum_{j=1}^{N} C_{j} f_{1 j} \\
\beta=4.1516 \times 10^{-4} \frac{\rho}{M \cdot E^{2}} \sum_{j=1}^{N} C_{j} f_{2 j}
\end{gathered}
$$

Since $\rho$ and $M$ can be directly linked to compositional coefficients $C_{j} \mathrm{~s} ; \delta$ and $\beta$ can be written as a function of compositional coefficients. 5 percent variation in the stoichiometry was analyzed which corresponded to 2.5 percent variation in the individual compositions of $\mathrm{Y}$ and Mn. It was assumed that the fractional compositions add up to a stiochiometric sample such that a Mn deficient sample is $\mathrm{Y}$ rich by the same amount. In other words, Mn or Y replaced each other in a non-perfect sample and the amount of oxygen stayed the same. The angular dependence curves are shown in Figure 26. As we can see, the curves show little sensitivity to the variation in refractive index. The reason for the insensitivity is apparent from the above equations. The composition sensitive terms $\rho, M$ and the summation terms, are scaled by a very small constant factor, $4.1515 \times 10^{-4}$. Thus, the variations in the stoichiometry have insignificant effect on $\delta$ and $\beta$. For $5 \% \mathrm{Mn}$ rich sample, the change in $\delta$ and $\beta$ is less than $0.1 \%$ and about $1 \%$, respectively, for $\mathrm{Mn} \mathrm{K}_{\alpha}$ energy. The changes in $\delta$ and $\beta$ for $\mathrm{Y} \mathrm{K}_{\alpha}$ energy are also of the same order of magnitude. Thus, the angular dependence was determined to be an 
insensitive technique for studying stoichiometry. The method of analyzing peaks ratio, that has a more direct dependence on the quantity of the element, was investigated. Also, the variation in the refractive index with difference with stoichiometry was ignored further on, based on the current discussion. 

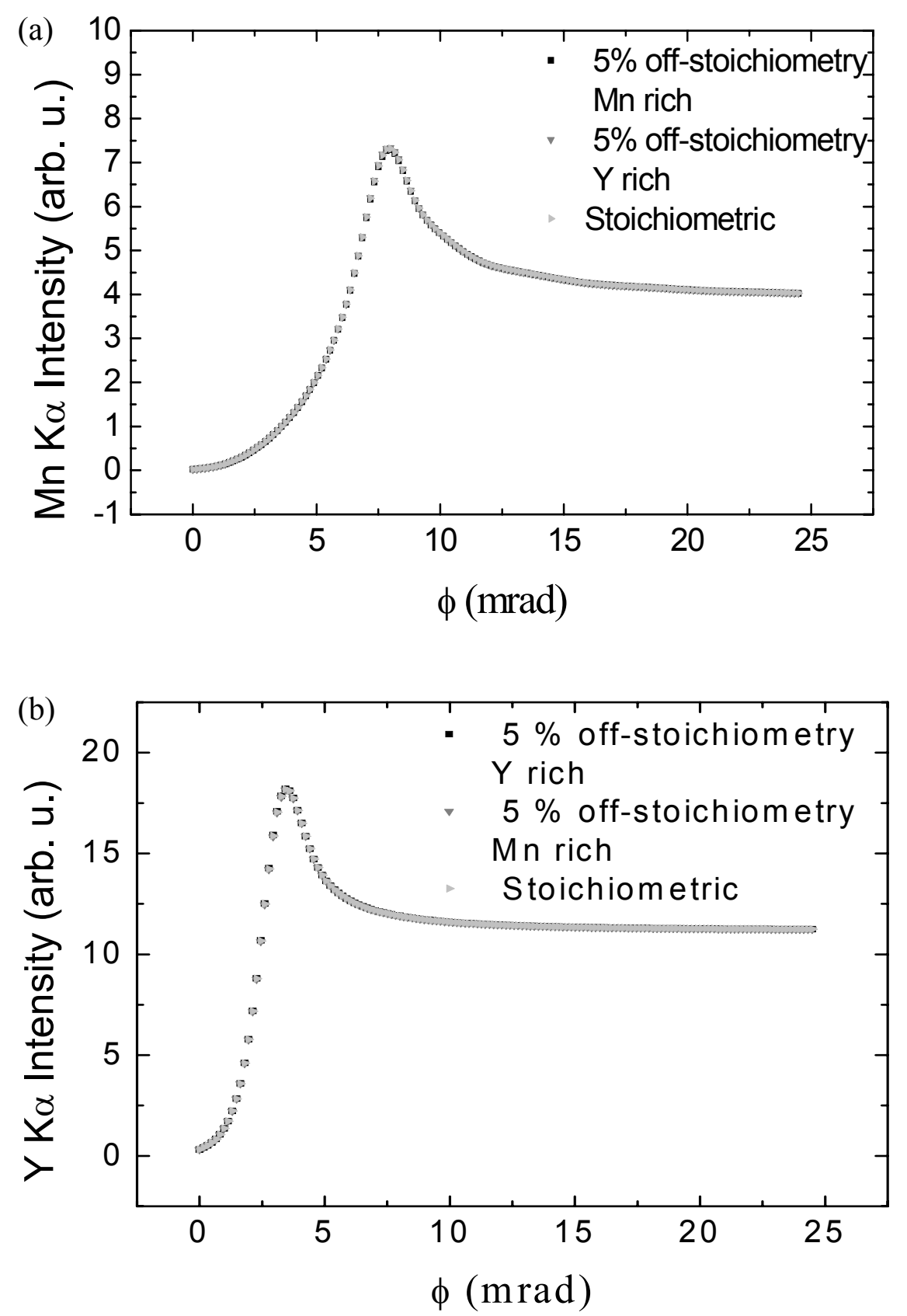

Figure 26

The variation in the angular dependence of $\mathrm{Mn}$ and $\mathrm{Y}$ fluorescence coming off $20 \mathrm{~nm}$ $\mathrm{YMnO}_{3}$ layer based on the variation in slight change in the refractive index. 


\subsection{Compositional analysis by peak ratio}

The major challenge in the stoichiometric growth of $\mathrm{YMnO}_{3}$ is the control of the relative compositions of $\mathrm{Y}$ and $\mathrm{Mn}$. Growth was performed under oxygen pressure in layer by layer fashion with the amount of $\mathrm{Y}$ and $\mathrm{Mn}$ for each layer based on a flux calibration using a quartz crystal monitor. If the relative composition of the two could be studied during growth, the shutter times could be adjusted in real time to compensate for the deficiency or excess of any material.

Since the fluorescence signal is directly proportional to the amount of the material, the ratio of $\mathrm{Y}$ and $\mathrm{Mn}$ signals at any angle, when compared with the ratio predicted for a stoichiometric compound, should yield the information about the relative compositions of the two. The complicating issue here, as mentioned before, was that the strength of the signal also depended on the thickness of the material. Simulations carried out to find out how the ratio at various angles changed with thickness showed that the ratio is more sensitive to the thickness of the film near the Mn and Y critical angles, but it is fairly constant away from the critical angle. Samples of different thicknesses were grown and their thickness values determined using x-ray reflectivity measurements. RHEEDTRAXS measurements were performed and $\mathrm{Y}$ to $\mathrm{Mn}$ ratios were then calculated at various angles and compared with the theoretical values using the sensitivity factor derived in the previous section.

The knowledge of the sample thickness and the relative yields of Mn and Y should help us associate a relative composition to an observed $\mathrm{Y}$ to $\mathrm{Mn}$ peak ratio. With this idea in mind, two thin Y/Mn bilayer samples were grown on a sapphire (001) substrate. First, an approximately $15 \mathrm{~nm}$ thick Mn layer was deposited on sapphire and the RHEED- 
TRAXS data was collected. Sapphire was used in order to eliminate the secondary fluorescence in $\mathrm{Mn}$ due to $\mathrm{Ga}$ in the regular GaN substrate. After performing RHEEDTRAXS measurements on the Mn layer, an approximately $15 \mathrm{~nm}$ thick layer of $\mathrm{Y}$ was deposited and the RHEED-TRAXS measurements were repeated. Y on top could not be fluoresced by $\mathrm{Mn}$ under it, so a good estimate of relative intensities due to electron excitation only could be obtained. Depositing Mn and Y on the same substrate ensured that the experimental conditions for both the layers were similar. The reproducibility of the conditions from sample to sample was extremely difficult to achieve as slight variations in the sample height and the rotation of the substrate manipulator could affect the count rate outside the error range. That's why the peak ratio was preferred over the absolute measurements that required reproducibility across samples. Once the samples were removed from the chamber, XRR measurements were performed on them after Al deposition to get a more accurate estimate of thickness value than those achievable by RHEED-TRAXS (Figure 27).

As discussed before, $\mathrm{Mn}$ has a much stronger signal than $\mathrm{Y}$ for the same experimental conditions and the same thickness of the film. This difference in signal strength was attributable to the difference in fluorescent yield and ionization crosssection of the elements and the difference in detector efficiency. These factors could be combined in a sensitivity factor $S$, dependent upon the element being investigated. To estimate $S$, the peak area ratios of $\mathrm{Y}$ and $\mathrm{Mn}$ at high angles, scaled by appropriate normalization factor based on experimental conditions and thickness values, were used. Normalization was carried out to estimate the relative response of $\mathrm{Y}$ and $\mathrm{Mn}$ atoms in terms of the peak area. Since it was known that at a high angle the fluorescence intensity 
is proportional of the thickness of the film for thin films, the following approach was adopted to estimate $S$.

Suppose the length of the sample illuminated by the electron beam was $l$, the beam width was $w$ and the thickness of the layer was $d$. Then the number of atoms of $Y$ or Mn contained in the volume lwd was proportional to $\left(\rho N_{a} / A\right) l w d$, where $\rho$ is the mass density of the material in $\mathrm{g} / \mathrm{cm}^{3}, N_{a}$ is the Avogadro's number and $A$ is the atomic mass. The normalized peak area $I^{\text {scaled }}(\phi)$ could be related to $I(\phi)$, the experimentally recorded area at an angle $\phi$, for $\mathrm{Mn}$ and $\mathrm{Y}$, as follows.

$$
\begin{aligned}
& I_{M n}^{\text {scaled }}(\phi)=\frac{I_{M n}(\phi)}{K_{M n}\left(\rho_{M n} N_{a} / A_{M n}\right) l w d_{M n}}, \\
& I_{Y}^{\text {scaled }}(\phi)=\frac{I_{Y}(\phi)}{K_{Y}\left(\rho_{Y} N_{a} / A_{Y}\right) l w d_{Y}}, \\
& K_{M n, Y} \propto i_{M n, Y} t_{M n, Y} .
\end{aligned}
$$

$K$ depended on the geometry of the setup, acquisition time $t$ and emission current $i$, $\rho_{M n}, \rho_{Y}$ are 7.3 and $4.46 \mathrm{~g} / \mathrm{cm}^{3}$, respectively, and the thickness of the layers was estimated using XRR.

The sensitivity factor $S$ could be calculated as

$$
S_{M n / Y}=\frac{I_{M n}^{\text {scaled }}(\phi)}{I_{Y}^{\text {scaled }}(\phi)}=\frac{I_{M n}(\phi)}{i_{M n} t_{M n}\left(\rho_{M n} / A_{M n}\right) d_{M n}} / \frac{I_{Y}(\phi)}{i_{Y} t_{Y}\left(\rho_{Y} / A_{Y}\right) d_{Y}} .
$$

Figure 28 shows the recorded $\mathrm{Mn}$ and $\mathrm{Y}$ peak areas for two $\mathrm{Y} / \mathrm{Mn}$ bilayer samples. Using the peak areas at high angles( $>26 \mathrm{mrad}$ for (a) and (b) and $26 \mathrm{mrad}$ for (c) and (d)) for the samples, the sensitivity factor was calculated as 


$$
S_{M n / Y}=2.71 \pm 0.08
$$

from the two samples analyzed.

Since the signal is independent of angle at high angles, scans at various high angles are equivalent to performing multiple scans at one of those positions. Assuming that the peak areas at various angles represent the samples from the same population, the standard error in the mean of peak area ratios was scaled by scaled by $1 / \sqrt{n}$ to obtain the error estimate reported in Equation 51.

This sensitivity factor obtained could be used to scale the relative intensities of $Y$ and $\mathrm{Mn}$ in $\mathrm{YMnO}_{3}$ sample and estimate their relative composition. Since the factor was reached without considering the effects of secondary fluorescence, the ratio obtained from $\mathrm{YMnO}_{3}$ sample should show some enhancement in the $\mathrm{Mn}$ signal and reduction in Y signal as some part of the $\mathrm{Y}$ fluorescence would excite Mn atoms. 

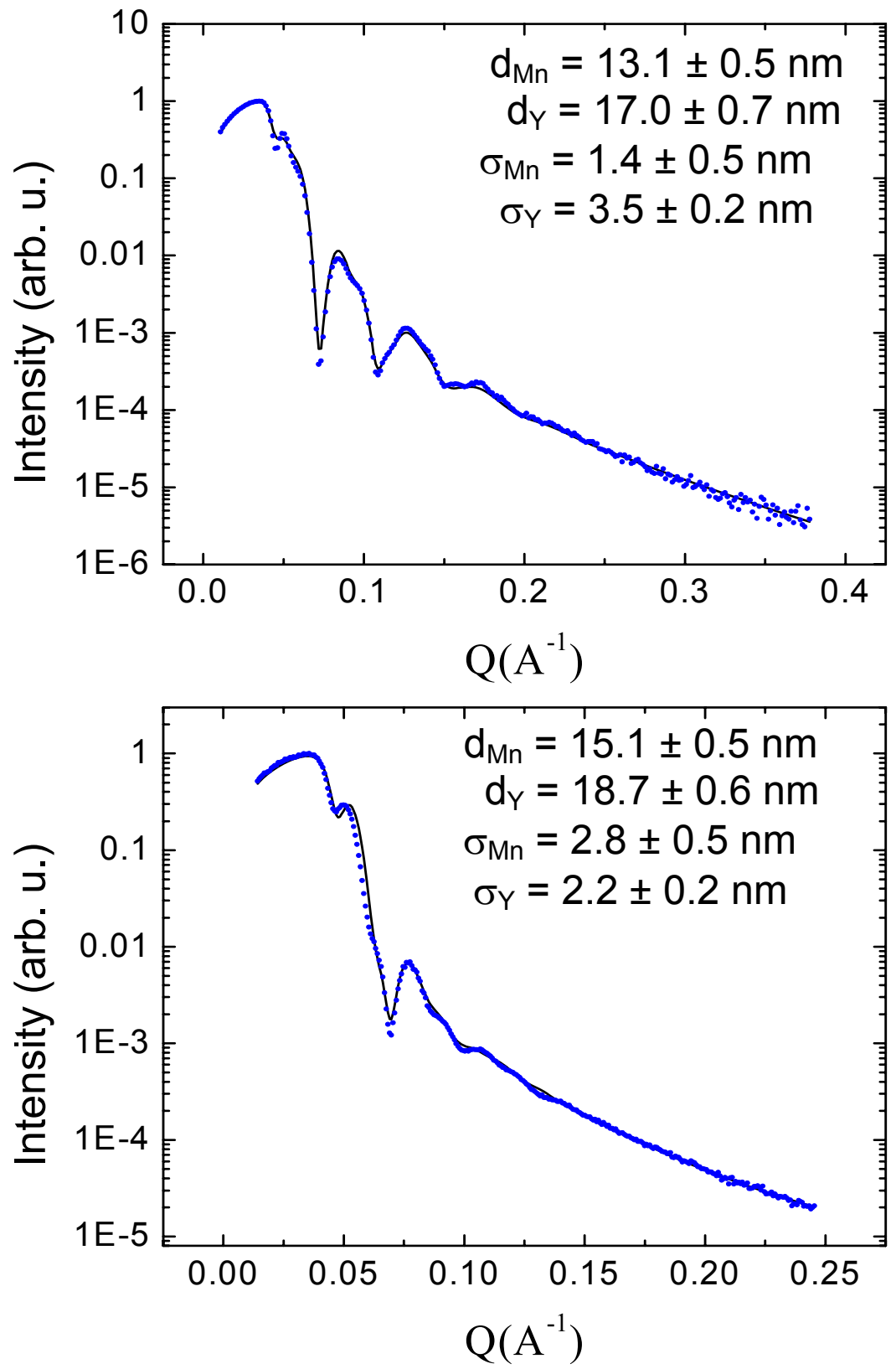

Figure 27

XRR fitting and thickness estimates for Y/Mn bilayer samples used for estimating relative sensitivity of $\mathrm{Mn}$ and $\mathrm{Y}$. Dots are the data and curve is the fit to the data using Parratt's model. 

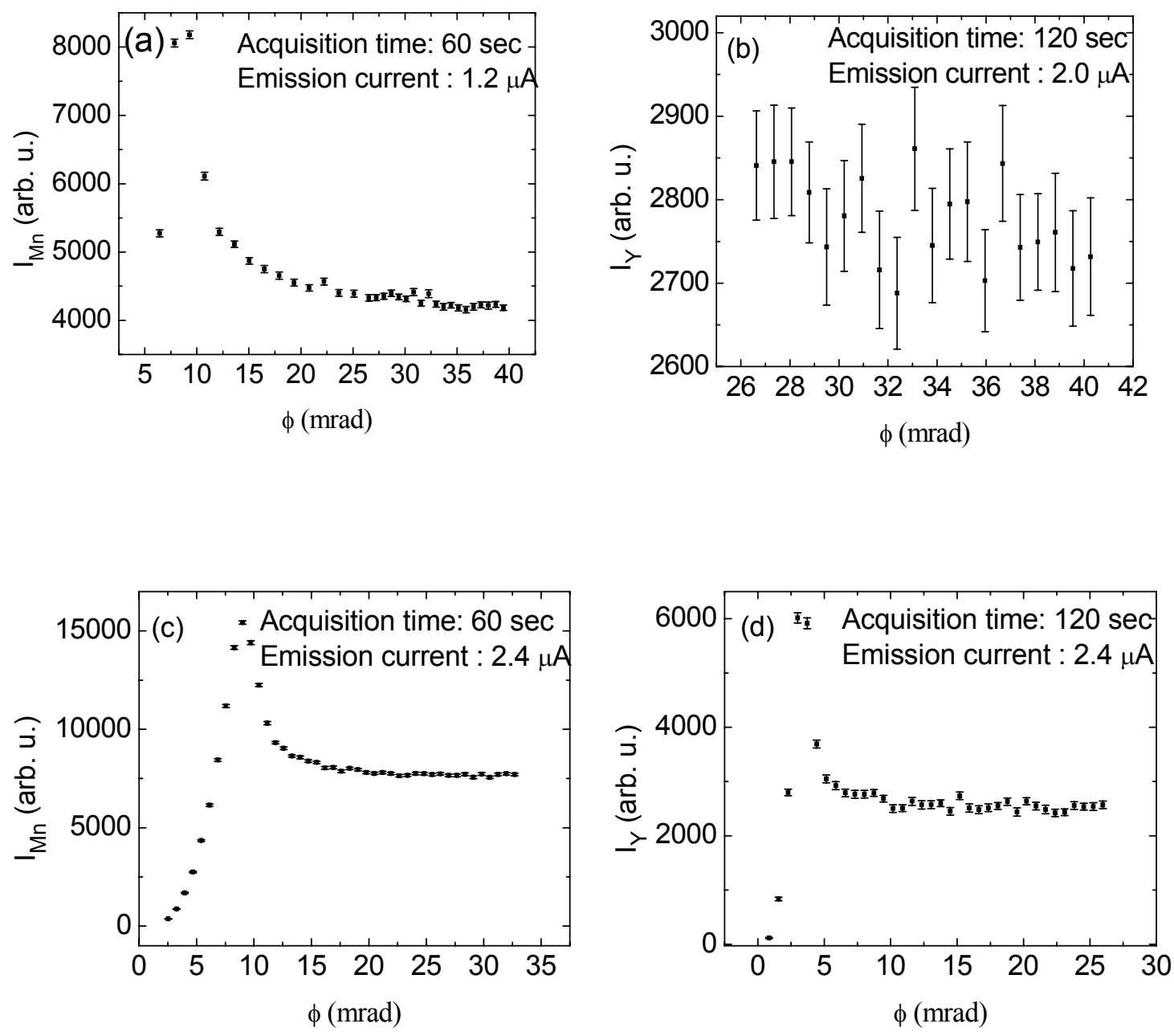

\section{Figure 28}

RHEED-TRAXS angular dependence for two Y/Mn bilayer samples. (a) and (b) are Mn and $\mathrm{Y}$ angular dependences for $13.1 \pm 0.5 \mathrm{~nm}$ thick $\mathrm{Mn}$ and $17.0 \pm 0.7 \mathrm{~nm}$ thick $\mathrm{Y}$ in first bilayer sample. Peak areas at angles greater than $26 \mathrm{mrad}$ were used to calculate the ratio in Equation 50. (c) and (d) are Mn and Y angular dependences for $15.1 \pm 0.5 \mathrm{~nm}$ thick Mn and $18.7 \pm 0.6 \mathrm{~nm}$ thick $\mathrm{Y}$ in the second bilayer sample. Peak areas greater at angles greater than $21 \mathrm{mrad}$ were used to calculate the ratio in Equation 50. 


\subsection{RHEED-TRAXS measurement on $\mathrm{YMnO}_{3}$}

RHEED-TRAXS measurements were performed on three $\mathrm{YMnO}_{3}$ samples of different thickness values and the results compared with RBS and XPS measurements. RHEED-TRAXS measurements were taken after removing the samples from the chamber and mounting them on a clean alumina block. The peak ratios were also calculated at various angles and compared with theoretical predictions after being scaled by the sensitivity factor described in the previous section. Comparing the results with RBS measurements yielded important information that could be used to study relative composition real time and possibly control the stoichiometry. XRR measurements were also performed to get a better estimate of the sample thickness values (Figure 29).

Figure 30 shows the theoretical curves for $\mathrm{Y}$ to $\mathrm{Mn}$ peak area ratios after considering the relative sensitivity for both elements, in the perfectly stoichiometric sample, and the experimental values obtained from three samples after being scaled by the sensitivity factor. The experimental data points in the figure were derived from Y and Mn peak areas using

$$
\text { Ratio }=\left.\frac{I_{Y}(\phi)}{I_{M n}(\phi)}\right|_{\text {Scaled }}=S_{M n / Y} \frac{I_{Y}(\phi)}{I_{M n}(\phi)}=(2.71 \pm 0.08) \frac{I_{Y}(\phi)}{I_{M n}(\phi)}
$$

The calculation for $S$ is discussed in the previous section and $I_{Y}(\phi), I_{M n}(\phi)$ are taken from the angular dependences shown in Figure 29. Thickness values used in Figure 30 are from XRR estimates shown is Figure 29. The experimental values match the theoretical curves at high angles, but at $3 \mathrm{mrad}$, which is the critical angle for $\mathrm{Y}$, all the values are below the theoretical predictions. This could be best understood by considering the secondary fluorescence effect and realizing, after RBS measurements, 

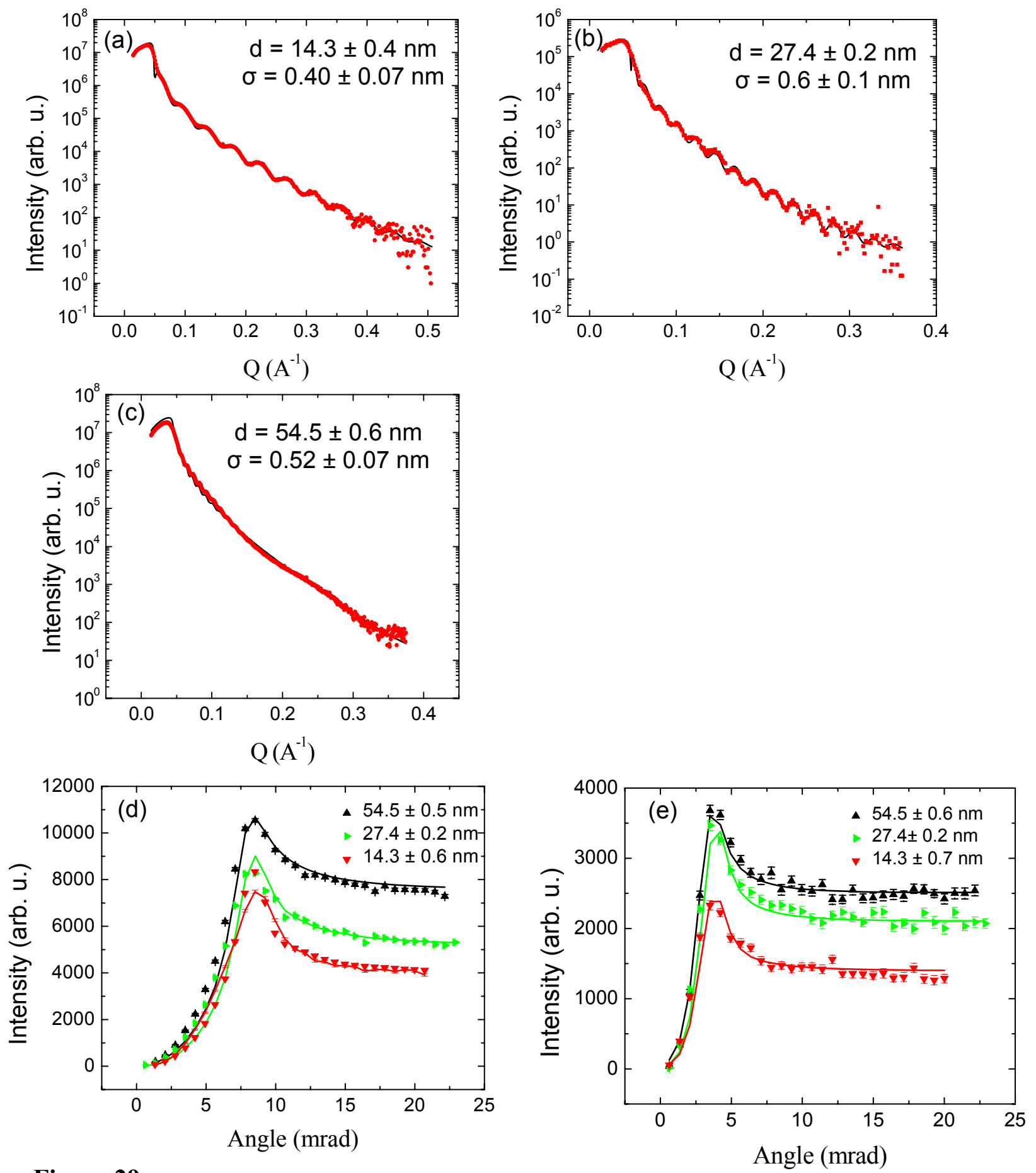

Figure 29

(a-c) XRR scans and best fits with layer thickness $d$ and roughness $\sigma$, (d-e) RHEED-

TRAXS scans and simulation based on the thickness values obtained by XRR for the 3 samples analyzed using RBS and XPS measurements. The layer thickness values are shown in the legend. 

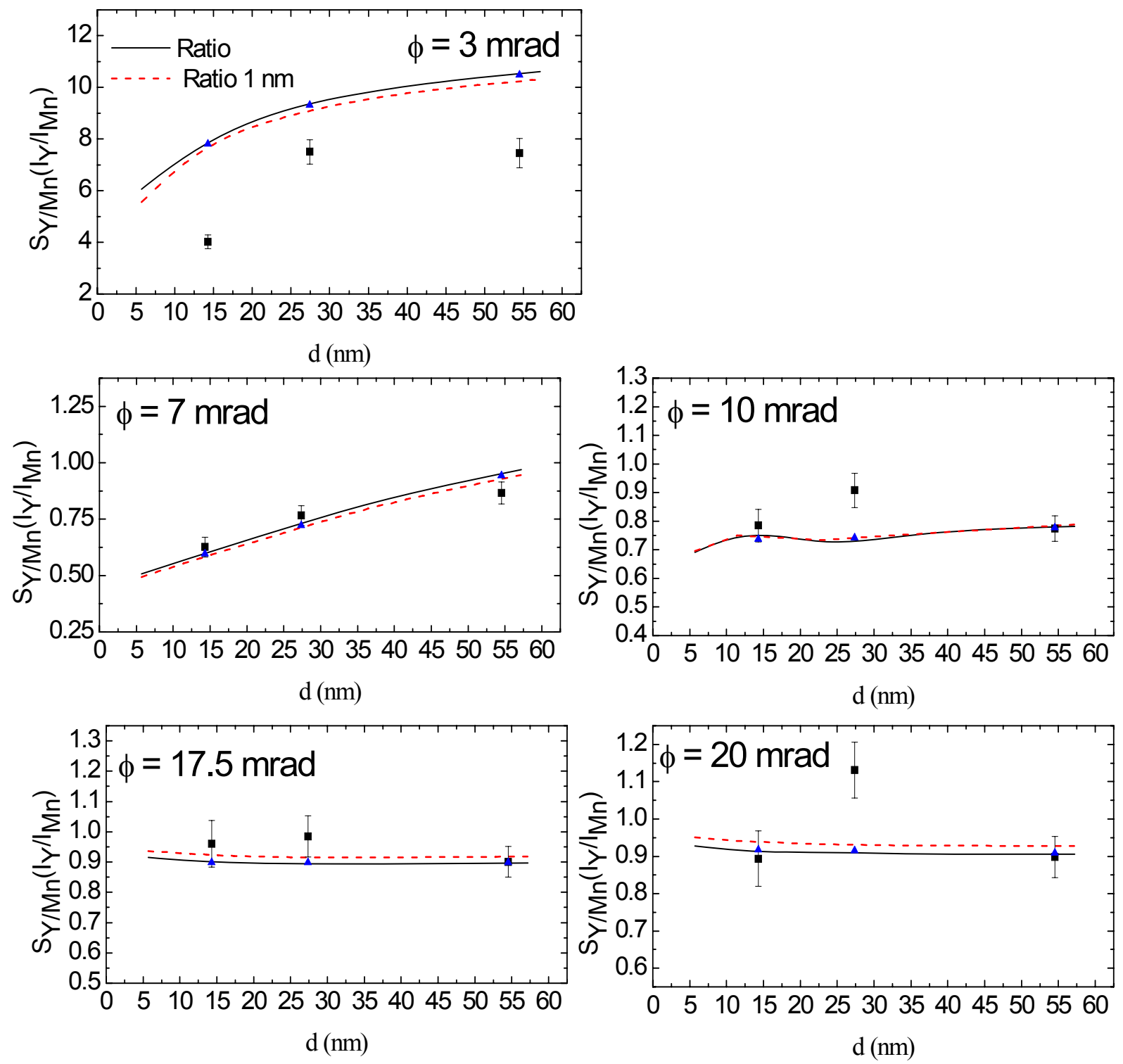

\section{Figure 30}

Theoretical curves and experimental data for the thickness dependence of $\mathrm{Y}$ to $\mathrm{Mn} \mathrm{K}_{\alpha}$ peak ratios at various angles in $\mathrm{YMnO}_{3}$ samples. The data points are reached upon by scaling the experimental peak ratios by the sensitivity factor as described in Equation 52 . The solid curves are for smooth samples, dashed curves are for a roughness of $1 \mathrm{~nm}$ and triangles are the simulation results using the real roughness values for the samples as determined using XRR measurements shown in Figure 28 (a)-(c). 
that all the samples are slightly $\mathrm{Y}$ rich. The sensitivity factor used to scale the experimental data points did not consider the attenuation of $\mathrm{Y}$ signal and the enhancement of Mn signal due to secondary fluorescence. Hence, it underestimated the $\mathrm{Y} / \mathrm{Mn}$ ratio from $\mathrm{YMnO}_{3}$. At $\phi=3 \mathrm{mrad}$, only a small region close to the surface was being probed, so the reduction in $\mathrm{Y}$ signal due to Mn excitation was significant. As more material is probed by moving to a higher angle, the excess $\mathrm{Y}$ in the film compensated for the secondary effects and moved the data points closer to the theoretical curves. In a perfectly stoichiometric sample, the experimental data points should be below the theoretical curves at all angles, as long as the same sensitivity factor is used. Nonetheless, the most important result was that at a high angle, where the critical angle peak for both materials has flattened out, the relative composition could be studied independently of the sample thickness by analyzing the peak ratio. This was an important result because it suggested a possibility of real time analysis.

As mentioned before, XPS measurements were performed on all three samples. The results for the $54.5 \mathrm{~nm}$ thick sample are shown in Figure 31. Figure 31(a) shows the wide survey scan to capture the whole spectrum. The scans were then narrowed down to the peaks of interest; $\mathrm{Y}, \mathrm{Mn}$ and $\mathrm{O}$, in our case. The carbon peak was due to sample surface contamination due to handling. Figure $31(\mathrm{~b}-\mathrm{d})$ shows the refined scan to better estimate the percent composition of the elements. The survey scan also revealed that there was a significant amount of boron contamination, possibly from the boron nitride crucible used in the Mn k-cell. After that, XPS measurement was also performed on a single layer of Mn to check for the presence of boron, but it showed no boron peak. This suggested that the boron incorporation in $\mathrm{YMnO}_{3}$ was, most possibly, related to the oxygen overpressure 

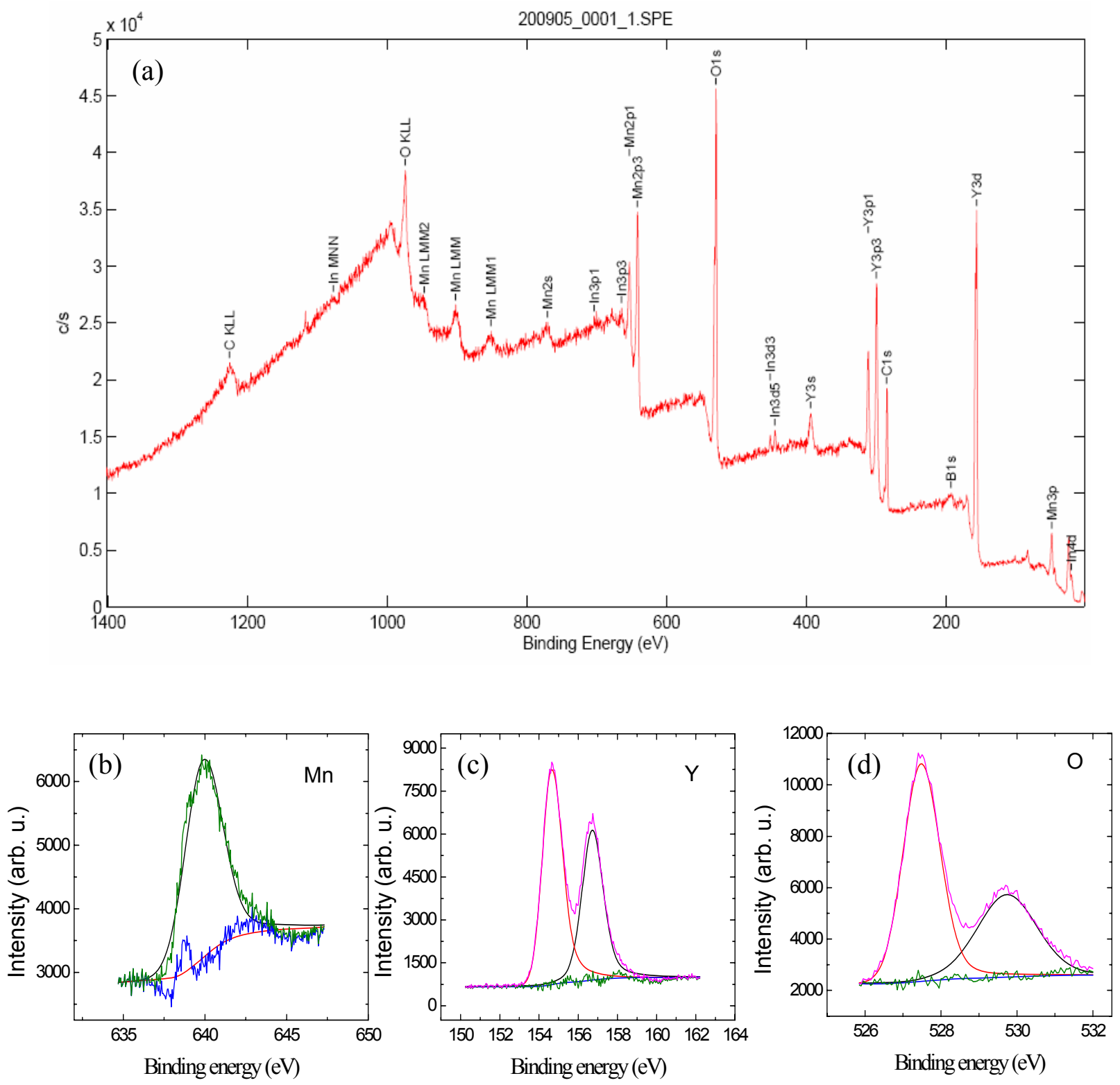

\section{Figure 31}

The XPS survey scan (a) and the detailed scans (b- d) of $54.5 \mathrm{~nm}$ thick $\mathrm{YMnO}_{3}$ sample used to determine the relative compositions of $\mathrm{Y}, \mathrm{Mn}$ and $\mathrm{O}$. The survey scan shows both photoelectric electrons and Auger electrons peaks. The peaks labeled as KLL, MNN and LMM are the Auger peaks. 

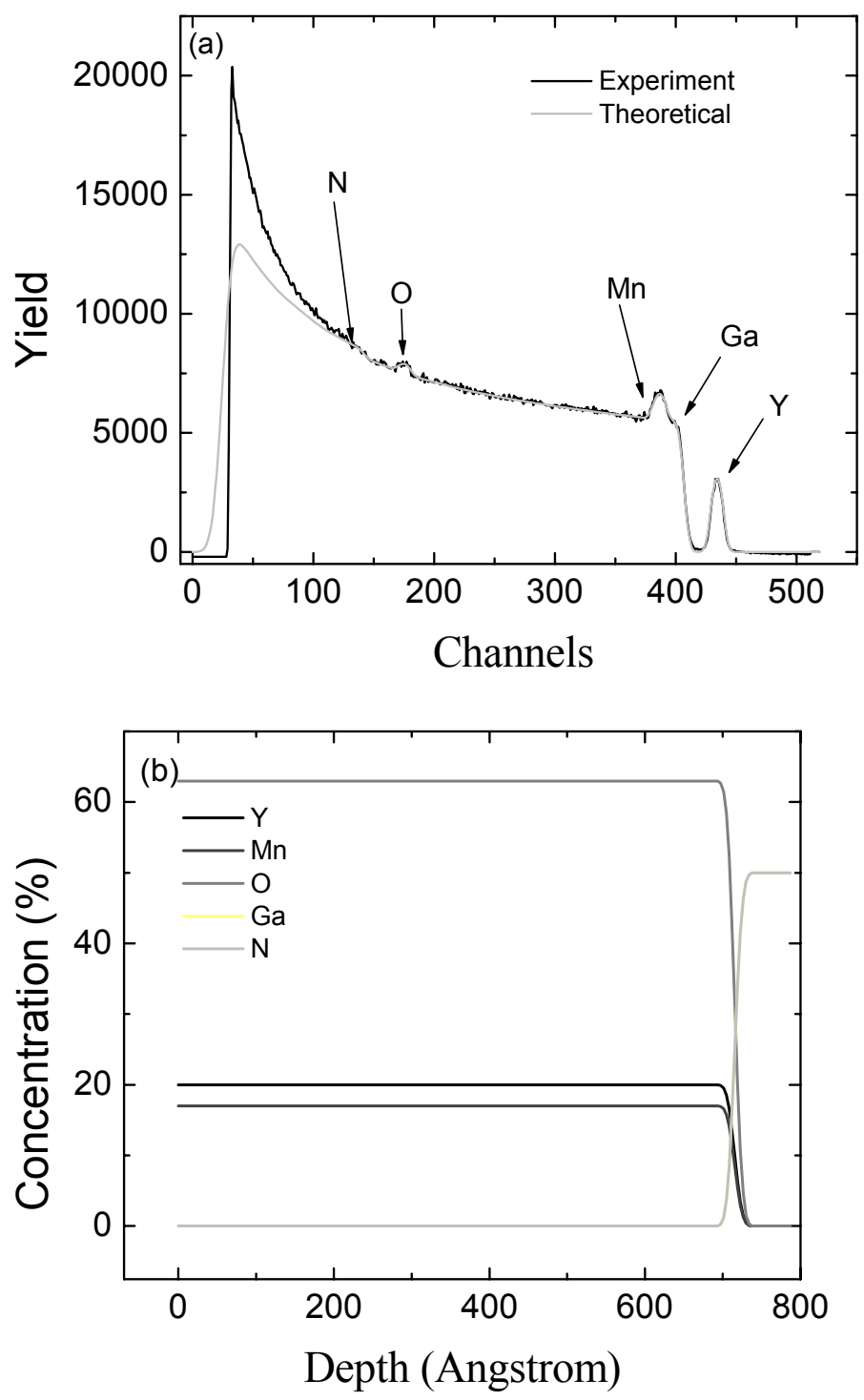

Figure 32

RBS results for $54.5 \mathrm{~nm}$ thick $\mathrm{YMnO}_{3}$ sample. (a) The yield of backscattered $\mathrm{He}++$ ions of $2.275 \mathrm{MeV}$ energy. The detector angle is $108^{\circ}$. The scaled intensities of various peaks give their relative compositions. (b) The relative compositions of $\mathrm{Y}, \mathrm{Mn}, \mathrm{O}, \mathrm{Ga}$ and $\mathrm{N}$ with depth. The given uncertainty in the estimates of $\mathrm{Y}, \mathrm{Mn}$ and $\mathrm{O}$ compositions is \pm 0.5 , \pm 1 and \pm 3 respectively. 
in the system during $\mathrm{YMnO}_{3}$ growth.

The relative compositions were deduced from XPS by scaling Y and Mn peak areas by the sensitivity factor found in a standard handbook. The sensitivity factor was based on the pure element standards and yielded a compositional ratio far off from the stoichiometry. According to XPS, the samples have almost twice as much Y as Mn. The ratios are summarized in Table 5. Since the RBS results which do not require any standards for calibration, yielded a ratio quite close to the stoichiometry, the only important feature of XPS results is the consistency in the trend, suggesting that all samples are close to the same stoichiometry point.

RBS has the capability of quantitative compositional analysis without the use of standards and yields information independent of the sensitivity factor for the element (as in XPS and RHEED-TRAXS). The results from RBS for one of the samples are shown in Figure 32. The results from RBS are also summarized in Table 5 along with RHEEDTRAXS results. The relative comparison shows that XPS does not have enough sensitivity to distinguish the differences in composition that are resolvable by both RBS and RHEED-TRAXS.

In Figure 33 (a), the ratio of $\mathrm{Y}$ to $\mathrm{Mn}$ peak areas is an average over 7 to 10 data points at high angles (>16 mrad) and has an uncertainty of about $1 \%$. So the total acquisition time for the reported uncertainties was of the order of 25 minutes, which is unsuitable for real time measurements. For a single two minute scan, the uncertainty is about $6 \%$ of the value. Figure 33 (b) shows a larger uncertainty because of the uncertainty in the scaling factor used. 
In Figure 33 (a) the extrapolated value for a perfectly stoichiometric sample came out to be $0.29 \pm 0.02$ (for RHEED-TRAXS ratio) and is shown by an open circle. The other two straight lines and triangles correspond to what the ratio should be as predicted by the relative sensitivity of $S_{M n / Y}=2.71 \pm 0.08$ or $S_{Y / M n}=0.37 \pm 0.01$ (see Equation 52) and that relative sensitivity $S_{Y / M n}$ corrected for the difference in the refractive index of $\mathrm{YMnO}_{3}$ for $\mathrm{Y}$ and $\mathrm{Mn}$ energies. From Figure 30, $\mathrm{Y}$ x-rays are absorbed more than $\mathrm{Mn} \mathrm{x}-$ rays, yielding a $\mathrm{Y} / \mathrm{Mn}$ ratio to smaller than one (0.91). Making that correction leads to the value of $0.34 \pm 0.01$ which is still higher than the $0.29 \pm 0.02$ as predicted by the linear fit to the data point. The difference between 0.29 and 0.34 is explainable by secondary fluorescence effects. Since, the relative sensitivity and refractive index corrections ignored the fluorescence of Mn by Y radiation, accounting for those effects would diminish the Y signal and increase Mn signal, so the ratio should decrease as suggested by the linear fit. Figure 33 (b) shows RHEED-TRAXS and RBS relationship after making relative sensitivity and refractive index corrections and it is clear from the graph and RHEED-TRAXS underestimated the $\mathrm{Y}$ concentration in all the cases.

Calculations can be performed to obtain a relative sensitivity factor $S_{Y / M n}$ theoretically. The response of a $\mathrm{Y}$ and $\mathrm{Mn}$ atom was estimated by considering the ionization cross-section of the element, fluorescent yield and the detector efficiency curve. The ionization cross-section was calculated as [45]

$$
Q=\frac{\text { const. }}{E_{c}^{2}} \frac{\ln U}{U},
$$


where $U=E / E_{c}, E$ is the exciting energy and $E_{c}$ is the critical energy of the peak emission. The fluorescent yield $\omega$ is described in Chapter 1 and the detector efficiency $\eta$ is tabulated at Reference 39 . The total response is

$$
r=Q \omega \eta,
$$

so the relative intensity of $\mathrm{Y}$ to $\mathrm{Mn}$ signal is

$$
S_{Y / M n}^{\text {Theory }}=\frac{Q_{Y} \omega_{Y} \eta_{Y}}{Q_{M n} \omega_{M n} \eta_{M n}}=0.23
$$

which does not agree with the experimental value of $0.34 \pm 0.02$. The reason for this discrepancy is still under investigation.

The most easily exploitable part of the analysis for real-time analysis is the RHEED-TRAXS peak ratio trend with RBS results. This suggests that while growing the samples, the detector angle could be set at a high angle and Mn and $\mathrm{Y}$ compositions can be adjusted according to the change in Y/Mn peak ratios. The results have shown that all the samples grown were $\mathrm{Y}$ rich suggesting that the possible desorption of Mn while growing was a key factor in producing a stoichiometric sample. The work is currently underway to systematically study the effect of various factors such as shutter times, substrate temperature and plasma flow on the real time peak ratio measurements with the aim of producing stoichiometric samples. 


\section{Table 5}

The comparative table for relative composition of $\mathrm{YMnO}_{3}$ samples. XPS results are off by a constant factor because of the sensitivity factor used and showed lower sensitivity than RBS and RHEED-TRAXS results. RHEED-TRAXS ratios are derived from the average of $\mathrm{Y}$ to $\mathrm{Mn}$ peak ratios at angles greater than $16 \mathrm{mrad}$, scaled by the sensitivity factor of $2.71 \pm 0.08$ for $\mathrm{Mn}$ to $\mathrm{Y}$ response derived in section 5.2 and correction factor of 1.1 for Mn to Y, to account for the difference in refractive index as shown in Figure 30.

\begin{tabular}{|c|c|c|c|}
\hline Thickness (nm) & \multicolumn{3}{|c|}{$\mathrm{Y} / \mathrm{Mn}$ compositional ratio } \\
\hline & XPS & RBS & RHEED-TRAXS \\
\hline $14.3 \pm 0.6$ & $2.1 \pm 0.4$ & $1.08 \pm 0.08$ & $0.96 \pm 0.05$ \\
\hline $27.4 \pm 0.2$ & $2.4 \pm 0.5$ & $1.3+0.1$ & $1.16 \pm 0.05$ \\
\hline $54.5 \pm 0.6$ & $2.4 \pm 0.5$ & $1.17 \pm 0.09$ & $0.98 \pm 0.04$ \\
\hline
\end{tabular}



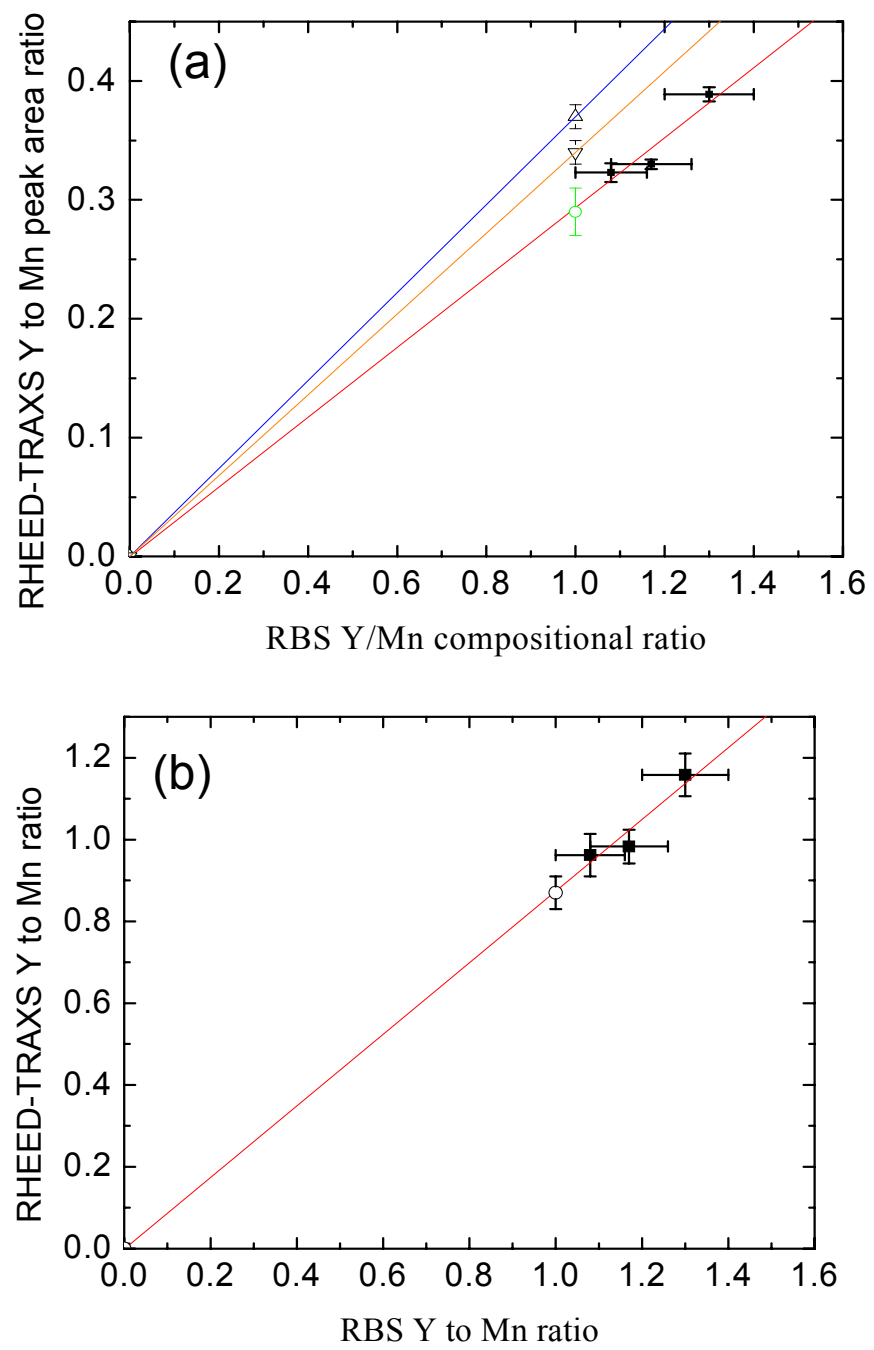

Figure 33

(a) shows the average of unscaled Y/Mn peak area ratios at angles greater than $16 \mathrm{mrad}$ for 3 samples. The solid lines are the linear fits. Open triangle at $(1,0.37)$ is based on relative sensitivity from bilayer samples, inverted triangle at $(1,0.34)$ is the relative sensitivity factor corrected for refractive indices for Mn and Y energies. Open circle is the stoichiometric point $(1,0.29 \pm 0.02)$ based on the linear fit to data point. (b) A linear fit to the RHEED-TRAXS and RBS results as shown in Table 5. The open circle at (1, $0.87 \pm 0.04)$, based on the linear fit, shows that RHEED-TRAXS underestimated the compositional ratio because of the ignored secondary effects. 


\section{Conclusion and Future Directions}

Previous work with RHEED-TRAXS have largely been qualitative and a lot less rigorous about the behavior of the x-rays in grazing exit regime. The current work demonstrated that the technique of RHEED-TRAXS can be used as a quantitative technique for in-situ analysis. The theoretical approach adopted simulated the behavior of fluorescence for thin layers of $\mathrm{Y}$ and $\mathrm{Mn}$ as well as bulk materials such as the GaN substrate. The structural results from single layers of $\mathrm{Mn}$ and $\mathrm{Y}$ were in agreement with the ex-situ results. For the bilayered structures, the estimates for the investigated layer were always good for the Mn signal, though the Y signal was small and yielded poorer estimates. For the compositional analysis, a stoichiometric film of $\mathrm{YMnO}_{3}$ would be very helpful for calibration, but reasonable estimates could be made by comparing the offstoichiometric samples with RBS measurements. Also, it's possible to perform real time measurement (acquisition time $\sim 1-2 \mathrm{~min}$.) by fixing the detector away from both the $\mathrm{Y}$ and Mn critical angle.

Trace element detection and compositional analysis by XRF is a standard technique and there are various instruments commercially available that are based on XRF analysis (Amptek Inc., Evans Analytical Group). An x-ray source is used for excitation and a standard is usually required for calibration. The reported errors are of the order of $1-2 \%$. The error in our peak ratio is about $5 \%$ for films that are a few nanometer thick for acquisition times of two to three minutes, which is a reasonable time interval to acquire data while growing a sample. A comparison of the sensitivity of $\mathrm{x}$-ray induced and electron induced XRF can be understood by comparing the attenuation length of x-rays 
inside the film. The attenuation length of $\mathrm{x}$-rays used as an excitation source for XRF is of the order of $100 \mu \mathrm{m}$, depending upon the x-ray target and the incidence angle. Hence, structures of the order of a few microns can also be probed. On the other hand, x-rays generated by RHEED electrons have the attenuation length of less than $10 \mathrm{~nm}$ (Figure 5).

If a copper target $\mathrm{x}$-ray source had been used at the same incidence angle as the RHEED gun, the attenuation length in a Mn film would have been $250 \mathrm{~nm}$. For RHEED-TRAXS, since x-rays are generated by electrons grazing the surface, the contribution of the signal coming from deeper inside the film is extremely diminished and leads not only to low sensitivity in the thickness measurements, but also larger uncertainty in the peak ratios. Hence, the errors in the technique are inherently larger than those encountered with techniques using $\mathrm{x}$-ray excitation sources and it is more suitable to probe layers of few nm thickness.

Structurally, the fluorescence from Mn or Y buried under Al layers could be used to characterize $\mathrm{Al}$ layers. This was a very useful aspect as the fluorescence from $\mathrm{Al}$ itself at $1.5 \mathrm{keV}$ was too low to be analyzed with the current setup. So the technique can be used to study the surface coverage of elements whose fluorescence signal can not be directly detected due to the detector efficiency limitations. The qualitative aspect of the changes in the angular dependence due to an overlayer can, in principle, be used to study growth kinematics. Al always caused the peaks to get sharper at the critical angle; Mn on $\mathrm{Y}$ also had a very dramatic opposite effect. Such qualitative effects can be picked up in relatively short acquisition time and can be used to study the migration of atoms perpendicular to the surface leading to effects such as precipitation, condensation or desorption of Mn atoms. 
For the theoretical treatment, the use of reciprocity theorem and distorted wave approximation simplified the problem as compared with the direct calculations, as suggested by Urbach et al. [46]. The kinematical approach, widely used in x-ray reflectivity analysis, could be used to develop a generalized model to handle multiple layered structures. The difference from the reflectivity approach was that instead of looking at the reflection from the top surface, the electric field inside the layer of interest was analyzed. Also, a strong depth dependence of the x-ray had to be considered.

The interaction of electrons inside the layered structure was simulated using CASINO. CASINO provided an easy and versatile way to produce the $\mathrm{x}$-ray depth profiles for the given experimental conditions. For a single layered sample, the simulation was carried out just once, so the computational needs were modest. For multilayered samples, the simulation was carried out iteratively using the thickness values from the angular dependence fitting routine. Though the first simulation was carried out with sufficiently thick layers, convergence between successive values was reached in less than 3 iterations. Good guess values for the initial thicknesses can lead to even faster convergence and eliminate the need for multiple simulations. Thus, the computational time for multilayered samples can also be reduced considerably.

Though the results from the work were positive, it also made us aware of the technological challenges towards that need to be overcome to realize full potential of the technique. A major technological hurdle that was overcome during the course of the study was the development of a current stabilizing device for the RHEED gun. It's a feedback device that reads the emission current and constantly corrects the filament current to keep the emission current constant. Constant emission was a necessary 
requirement for accurately acquiring the angular dependence data that might take a few hours. Though the emission current was stable, the stability and reproducibility of the beam position was less than ideal and a few experiments had to be aborted because of beam drifting.

Another issue that needs to be addressed is the strength of the signal. The signal was weak in general and even more so for $\mathrm{Y}$, rendering the data acquisition time unsuitable for real-time analysis. This was also because the detector sat too far from the sample and the analysis required a range of angles be scanned. In its current state, it can not be used to do useful analysis during growth when surface conditions change quickly. One solution is to put the detector close to the sample to increase the count rate and cut the acquisition time at every angle to a few seconds. The detector translation stage can also be automated to scan at a faster rate. As for now, the detector position is changed by hand after every reading. Another advantage of getting a higher count rate is that the slit width can be decreased for better resolution and it is possible to use a much faster silicon drift detector (SDD) which is capable of handling much higher count rates. The count rate capabilities of a SDD can outperform a conventional Si detector by a factor of 10 . Also, another possibility is to use a position sensitive detector that can collect the whole angular dependence data simultaneously.

The effects of secondary fluorescence were not considered in the analysis so far as CASINO does not incorporate that effect into the x-ray depth profile. With the improved setup capable of performing more precise experiments, the effect of the secondary fluorescence could possibly be observed and analyzed. PENELOPE2000 is a potential software for analyzing electron-matter interaction that does account for the secondary 
fluorescence effects. But it was under testing by Radiation Safety Information Computational Centre (RSICC) and was not released for public use when this work was performed. In summary, the technique has potential for a wide applicability and a lot of room for improvement from a technological as well as theoretical point of view. 


\section{REFERENCES}

[1] H. Kiessig, Ann. Phys. (Leipzig) 10 (1931) 769.

[2] L. G. Parratt, Phys. Rev. 95, 359 (1954).

[3] Y. Yoneda, T. Horiuchi, Rev. Sci. Instrum. 42, (1971) 1069

[4] R.S. Becker, J. A. Golovchenko, and J. R. Patel, Phys. Rev. Lett. 50, 153 (1983).

[5] T. W. Barbee Jr. and W. K. Warburton, Mater. Lett. 3, 17 (1984).

[6] S. Hasegawa, S. Ino, Y. Yamamoto, H. Daimon, Jpn. J. Appl. Phys., 24, L387(1985).

[7] J. Shigetomi et al. J. Cryst. Growth 111, 110 (1991).

[8] M. Kamei et al. Jpn. J. Appl. Phys. 74, 436 (1993).

[9] Y. Aoki, M. Kamei, S. Ogota, T. Usui, T. Morishita, IEEE Trans. Appl.

Superconductivity, 3, 1598(1993)

[10] J. G. Pellegrino, J. Armstrong, J. Lowney, B. DiCamillo, J. C. Woicik, Appl. Phys. Lett., 73, 3580(1998)

[11] W. Braun, K. H. Ploog, J. Cryst. Growth, 251, 68 (2003).

[12] W. B. Yun, J, M, Bloch, J. Appl. Phys., 68, 1421 (1990).

[13] K. Tsuji, K, Tctsuoka, e-J Surf. Sci. Nanotech. 1, 111 (2003).

[14] K. Tsuji, K. Hirokawa, Spectrochima Acta, 48B, 1471 (1993).

[15] K. Tsuji, S. Sato, K. Hirokawa, J. Appl. Phys., 76, 7860 (1994)

[16] K. Tsuji, K. Hirokawa, J. Appl. Phys., 75, 7189(1994).

[17] T. P. Trainor, A. S. Templeton, P. T. Eng, J. electron spectros. 150, 66 (2006).

[18] D. K. G. de Boer, A. J. G. Leenaers, W. W. van den Hoogenhof, X-ray Spectroscopy, 24, 91(1995).

[19] T. Noma, H. Miyata, S. Ino, Jpn. J. Appl. Phys., 31, L900(1992). 
[20] S. K. Ghose, B. N. Dev, Phys. Rev. B, 63,245409 (2001).

[21]P.K. de Bokx and H.P. Urbach, Rev. Sci. Instrum. 66, 15 (1995).

[22] M. Born and E. Wolf, Principle of optics (Cambridge University Press, Cambridge 1999).

[23] G. H. Vineyard, Phys. Rev. B 26, 4146 (1982).

[24] V. D. Scott, G. Love and S. J. B. Reed, Quantitative Electron-Probe Microanalysis, pp. 212-238, (Ellis Horwood, London, 1995).

[25] J. A. Small, D. E. Newbury, and J. T. Armstrong,

Handbook of X-ray Spectrometry, 2nd ed., 811-931 (Marcel Dekker, New York, 2002).

[26] R. Klockenkamper, Total reflection X-ray Fluorescence Analysis (WileyInterscience, 1996).

[27] D. Drouin, et. al. Scanning 29, 92 (2007).

[28] H. A. Bethe 1933 Handbuch der Physik (Springer, Berlin, 1933)

[29] P. Hovington, D. Drouin, R. Gauvin, Scanning 19, 1 (1997)

[30] J. Brown, Electron Beam Interactions with Solids, 137 (1984).

[31] J. Philibert, 3rd Int. Sum. on X-Ray Optics and Microanalysis (Academic Press, N.Y., 1963)

[32] Y. C. Sasaki, K. Hirokawa, Appl. Phys. Lett. 58, 1384 (1991).

[33] K. Tsuji, A. Sasaki, K. Hirokawa, Jpn. J. Appl. Phys, 33, 6316(1994).

[34] K. Tsuji, T. Yamada, T. Utaka, K. Hirokawa, J. Appl. Phys. 78, 969(1995).

[35] S. Krassimir, K. Sakurai, The Rigaku Journal 14, 22 (1997).

[36] P. Croce, L. Nevot, Revue Phys. Appl., 11, 113(1976)

[37] L. Nevot, P. Croce, Revue Phys. Appl., 15, 76191980) 
[38] L. Nevot, B. Pardo, J. Corno, Revue Phys. Appl., 23, 1675(1988)

[39] L. D. Feranchuk, A. A. Minkevich, A. P. Ulyanenkov, Eur. Phys. J. Appl. Phys, 24, $21(2003)$.

[40] www.amptek.com (2009).

[41] Origin (Version 7.5), (C1991-2006, OriginLab Corporation.

[42] Paratt32 (Version 1.6.0), (C1997-99, Christian Braun, HMI Berlin.

[43] http://www.uksaf.org/tutorials.html (2009)

[44] http://www.cea.com/publications/literature.php (2009)

[45] G. Love, V. D. Scott, Scanning, 4, 111(1981).

[46] H. P. Urbach, P. K. de Bokx, Phys. Rev. B, 63, 085408(2001).

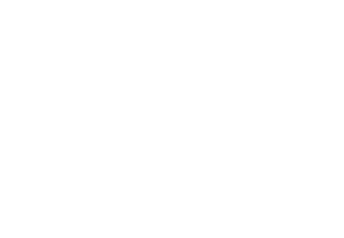

